\title{
Achilles Tendinopathy
}

\section{Evaluation and Treatment}

\author{
Karin Grävare Silbernagel
}

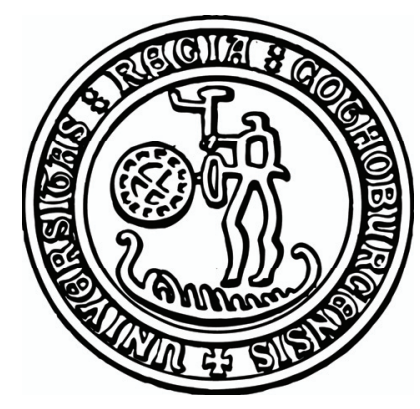

Department of Orthopaedics Institute of Clinical Sciences

The Sahlgrenska Academy at Göteborg University

Göteborg, Sweden 
(C) Karin Grävare Silbernagel

Front cover and figure 1-4, 7, 10, and 27: Illustrations by Annette Dahlström

ISBN 91-628-6764-4 
"The art of medicine consists in amusing the patient while nature cures the disease" Voltaire (1694-1778) 


\section{Contents}

Abstract 6

List of publications 8

Abbreviations and definitions 9

Introduction 12

The normal Achilles tendon 13

Anatomy 13

Tendon structure 14

Circulation 16

Innervation 16

Metabolism 17

Biomechanics 17

Effect of exercise on tendons 21

Effect of immobilization on tendons 22

Effect of remobilization and reconditioning on tendons 23

Effect of age on tendons 23

Healing process in tendons 24

Achilles tendon injury 25

Classification of Achilles tendon injury 26

Classification of tendinopathies 28

Achilles tendinopathy 29

Epidemiology 29

Etiology 30

Pathogenesis 33

Pathophysiology 35

Differential diagnoses 35

Evaluation 36

Treatment 45

Prevention 49

Prognosis 50

Summary of problem areas presented in the introduction 51

Aims of thesis 52

Subjects 53

Ethics 57

Methods 58

Evaluation 58

Treatment 72

Statistical methods 79

Summary of studies 81 
Discussion 87

Subjects 87

Evaluation 90

Treatment 95

Clinical recommendations 100

Limitations 101

Conclusions 102

The future 104

Abstract in Swedish 105

Acknowledgements 107

References 110

Appendices 121

VISA-A-S questionnaire

VISA-A questionnaire

Papers I-V 


\title{
Achilles Tendinopathy \\ Evaluation and Treatment \\ Karin Grävare Silbernagel
}

\begin{abstract}
.
Background: Achilles tendinopathy is considered to be one of the most common overuse injuries in elite and recreational athletes. There are, however, only a few randomized studies of treatment and there is a need for standardized outcome measures for the patients' symptoms and function.

Purpose: The overall purpose of this thesis was to develop and evaluate outcome measures and treatment protocols for patients with Achilles tendinopathy.
\end{abstract}

Material, Methods and Results: Initially, a method was developed to evaluate both symptoms and function in Studies I and II. The method was shown to have good reliability in 10 patients with acute Achilles tendon injuries and in 32 patients with chronic Achilles tendinopathy. We questioned, however, the validity of the methods since only small changes were detected while symptoms improved. At that time there was no standardized symptom questionnaire for the assessment of patients with Achilles tendinopathy. A questionnaire of this kind became available in 2001 in English. In Study III this questionnaire, the Victorian Institute of Sports Assessment - Achilles questionnaire (VISA-A), was cross-culturally adapted and evaluated for reliability, validity and structure on 15 healthy subjects and 51 patients. The Swedish version of the VISA-A questionnaire (VISA-A-S), which measured two factors, pain/symptoms and physical activity, was shown to have good reliability and to be a valid instrument, fully comparable to the original version. The VISA-A-S can be used in both research and clinical settings.

A test battery for lower leg muscle/tendon function, including jump and strength tests, was developed and evaluated on 42 patients in Study IV. The purpose of the test battery was to evaluate, in more detail than had previously been possible, whether Achilles tendinopathy caused functional deficits on the injured side compared with the non-injured side. The test battery was found to be reliable and able to detect clinically relevant differences in lower leg function between the injured or "most symptomatic" and non-injured or "least symptomatic" sides in patients with Achilles tendinopathy. The test battery imposed more rigorous demands on patient function compared with each individual test. 
Treatment comprising iontophoresis using dexamethazone combined with exercise for patients with acute Achilles tendinopathy was evaluated on 25 patients in a randomized, double-blind design in Study II. Positive effects on symptoms were found from using iontophoresis with dexamethazone, compared with a control group in patients with acute Achilles tendon pain.

Achilles tendon and calf muscle strengthening exercises (intensity modified with the use of a pain-monitoring model) as treatment for patients with chronic (symptoms for more than 2-3 months) Achilles tendinopathy was evaluated in 40 patients in Study I and in 38 patients in Study V. Furthermore, the effect of continued running and jumping on treatment outcome was evaluated in Study V. A treatment protocol which includes Achilles tendon and calf muscle exercises resulted in significant improvements in patients with chronic Achilles tendinopathy. When the painmonitoring model was used, no negative effects could be demonstrated from continuing Achilles tendon loading activity (such as running and jumping) during treatment.

Conclusion: The VISA-A-S questionnaire can be used to evaluate the clinical severity of patients with Achilles tendinopathy and it is useful in both research and clinical settings. Patients with Achilles tendinopathy reports not only pain, but also demonstrate deficits in lower leg function. In the acute phase, the use of iontophoresis with dexamethazone could potentially be beneficial. For patients with both acute and chronic Achilles tendinopathy, the performance of Achilles tendon and calf muscle strengthening exercises with the use of a pain-monitoring model for 6 months can be recommended. A training regimen of continued, pain monitored, Achilles tendon loading physical activity, such as running and jumping, might represent a valuable option for patients with Achilles tendinopathy.

Key words: Achilles tendinopathy, Achilles tendon, Victorian Institute of Sports Assessment - Achilles questionnaire (VISA-A), VISA-A-S, functional evaluation, treatment protocol, strengthening exercise, pain monitoring model, test battery, iontophoresis, prospective, randomized 


\section{List of papers}

This thesis is based on the following papers, which are referred to by their Roman numerals.

I. Grävare Silbernagel K, Thomeé R, Thomeé P, Karlsson J. Eccentric overload training for patients with chronic Achilles tendon pain - a randomised controlled study with reliability testing of the evaluation methods.

Scand J Med Sci Sports 2001;11:197-206

II. Neeter C, Thomeé R, Grävare Silbernagel K, Thomeé P, Karlsson J. Iontophoresis with or without dexamethazone in the treatment of acute Achilles tendon pain.

Scand J Med Sci Sports 2003;13:376-382

III. Grävare Silbernagel K, Thomeé R, Karlsson J. Cross-cultural adaptation of the VISA-A questionnaire, an index of clinical severity for patients with Achilles tendinopathy, with reliability, validity and structure evaluations.

BMC Musculoskeletal Disorders 2005,6:12

IV. Grävare Silbernagel K, Thomeé R, Gustavsson A, Karlsson J. Evaluation of lower leg function in patients with Achilles tendinopathy Accepted for publication in Knee Surgery, Sports Traumatology, Arthroscopy 2006

V. Grävare Silbernagel K, Thomeé R, Eriksson BI, Karlsson J. Continued sports activity, using a pain monitoring model, during rehabilitation in patients with Achilles tendinopathy - a randomized controlled study. Manuscript 


\section{Abbreviations and definitions}

In the present thesis, the following abbreviations and definitions were used

\section{CMJ}

Concentric muscle action

Construct validity

Content validity

Criterion validity

Drop CMJ

Eccentric muscle action

Epidemiology

Etiology

Extrinsic

Factor analysis

FAOS

Hopping

ICC
Countermovement jump

When a muscle shortens while producing force

Psychometric property of an outcome instrument assessing whether the instrument follows accepted hypotheses (constructs) (Kocher and Zurakowski, 2004)

Psychometric property of an outcome instrument assessing whether the instrument is representative of the characteristic being measured (face validity) (Kocher and Zurakowski, 2004) Psychometric property of an outcome instrument assessing its relationship to an accepted, "gold standard" instrument (Kocher and Zurakowski, 2004).

Drop countermovement jump

When a muscle lengthens while producing force

The branch of medicine that deals with the study of the causes, distribution and control of disease in populations The branch of medicine that deals with the causes or origins of disease

Originating from the outside; external to the patient

Statistical method for analyzing relationships among a set of variables to determine underlying dimensions Foot and Ankle Outcome Score A continuous rhythmical jump, similar to jumping rope Intra-class correlation coefficient 
Incidence

Internal consistency

Intrinsic

LSI

MRI

Nonparametric methods

Pathogenesis

Pathophysiology

Plyometric quotient

Power

Prevalence

Reliability

Risk factor

ROM
The extent or rate of occurrence, especially the number of new cases of a disease in a population over a period of time Psychometric property of an outcome instrument regarding the degree to which individual items are related to each other (Kocher and Zurakowski, 2004) Of or relating to the essential nature of a thing; inherent, relating to the patient Limb Symmetry Index. The LSI is defined as the ratio of the involved limb score and the uninvolved limb score expressed in percent (involved/uninvolved x $100=$ LSI) Magnetic Resonance Imaging Statistical tests making no assumption regarding the distribution of data (Kocher and Zurakowski, 2004)

The development of a diseased or morbid condition

The functional changes associated with or resulting from disease or injury

Flight time divided by contact time

1. The rate of performing work; the product of force and velocity (SI unit: watt)

2. Probability of finding a significant association when one truly exists (1 probability of type-II error) (Kocher and Zurakowski, 2004)

The total number of cases of a disease in a given population at a specific time Measure of reproducibility of a measurement (Kocher and Zurakowski, 2004)

Something that increases risk or susceptibility

Range of motion 
Sensitivity

SJ

Specificity

SSC

Toe raise

US

Validity

VAS

VISA-A questionnaire

VISA-A-S questionnaire

Work
Proportion of patients who have the outcome who are classified as having positive results (Kocher and Zurakowski, 2004)

Squat jump

Proportion of patients without the outcome who are classified as having negative results (Kocher and Zurakowski, 2004)

Stretch-Shortening Cycle

The exercise where the person goes up onto the toes (performing ankle plantarflexion when standing) and back down

\section{Ultrasonography}

Degree to which a questionnaire, instrument or test measures what it is intended to measure Visual Analogue Scale Victorian Institute of Sports Assessment Achilles questionnaire The Swedish version of the VISA-A questionnaire The product of the force and the distance through which the body moves and it is expressed in joules 


\section{Introduction}

The human body is built for activity and movement and not for sitting in a sedentary position in front of computers. We are progressively becoming less active in our daily lives and instead try to fulfill our bodies' demand for exercise in short spurts. This irregular activity pattern is the breeding ground for overuse types of injury.

Studies at cellular level, as well as clinical treatment studies, indicate that a mechanical load on structures is vital in order to maintain healthy, strong tissues, as well as for healthy life in humans. The body needs to be challenged in order to respond with improved function. Exercise as treatment is suitable not only for musculoskeletal injuries but also for metabolic syndrome-related disorders, heart and pulmonary disease, coronary heart disease, chronic heart failure, intermittent claudication, cancer, depression, asthma and type 1 diabetes (Pedersen and Saltin, 2006). The challenge in today's society is to find a healthy balance between our sedentary lifestyle and the body's need for physical activity. General inactivity during the week, followed by extreme exercise at weekends, allows for overuse types of injury. We need to realize that the gradual progression of loading in all tissues is vital for humans.

It has been estimated that $30-50 \%$ of all sports injuries are so-called overuse injuries (Järvinen, 1992, Kannus, 1997b). Of all sports injuries, tendon injuries have become a major problem in competitive and recreational sports (Kannus, 1997b). It has also been estimated that chronic tendon injuries account for approximately 50\% of occupational illnesses (Almekinders and Temple, 1998). The Achilles tendon is one of the most injured tendons especially in athletes involved in running and jumping (Kvist, 1994, Józsa and Kannus, 1997, Alfredson and Lorentzon, 2000, Stanish et al., 2000, Paavola et al., 2000a). Achilles tendinopathy is, however, not always related to excessive physical activity. It has been found that about one third of patients with Achilles tendinopathy do not participate in vigorous physical activity (Rolf and Movin, 1997).

Injuries to the Achilles tendon, especially Achilles tendinopathy, cause many patients to significantly reduce their physical activity level, with a potentially negative impact on their overall health and general well-being (Kvist, 1994, Józsa and Kannus, 1997, Paavola et al., 2002a).

My goal with this thesis was to improve clinical evaluations and treatment for patients with Achilles tendinopathy, so that they can continue to be physically active. 


\section{The normal Achilles tendon}

\section{Anatomy}

The gastrocnemius and soleus muscles are known as the triceps surae and are the main plantarflexors of the ankle (Norkin and Levangie, 1983). There are two heads of the gastrocnemius - one originating one from the medial and one from the lateral condyle of the femur - while the soleus originates from the upper third of the fibula and central third of the tibia (Humble and Nugent, 2001). The Achilles tendon, which is the largest tendon in the body, is the common tendon of these muscles and it inserts into the most posterior aspect of the calcaneus (Figure 1).

The muscle fibers of the gastrocnemius extend 11-26 $\mathrm{cm}$ above the calcaneus, while the soleus muscle fibers extend $3-11 \mathrm{~cm}$ above the calcaneus (Jones, 1998). The Achilles tendon attachment to the calcaneus is far from the ankle axis and provides a large moment arm for plantar flexion (Norkin and Levangie, 1983). The triceps surae muscle crosses both the knee and ankle joints and is therefore involved in both knee and ankle motions. The soleus muscle has a stabilizing effect on the foot and is continuously active during erect standing (Norkin and Levangie, 1983). Various positions of the knee and ankle joints can affect the forces along the Achilles tendon (Arndt et al., 1998, Davis et al., 1999). The Achilles tendon is broad and flat where it originates and becomes more narrow and rounded distally before it fans out at the insertion (Józsa and Kannus, 1997). As the Achilles tendon descends, the tendon fibers rotate up to 90 degrees laterally, which means that the dorsal fibers rotate anterolaterally and the ventral fibers rotate posteromedially (Józsa and Kannus, 1997). Clinically, it is also important to distinguish between the Achilles tendons proximally - musculotendinous junction, midportion of tendon - and distally - osteotendinous junction (figure 7). In the proximal part there are combinations of tendon and muscle tissue, in the midportion there is only true tendon tissue and, distally in the insertion into the calcaneus, there is a successive change from tendon tissue to fibrocartilage and finally to lamellar bone (Józsa and Kannus, 1997, O'Brien, 1997). 


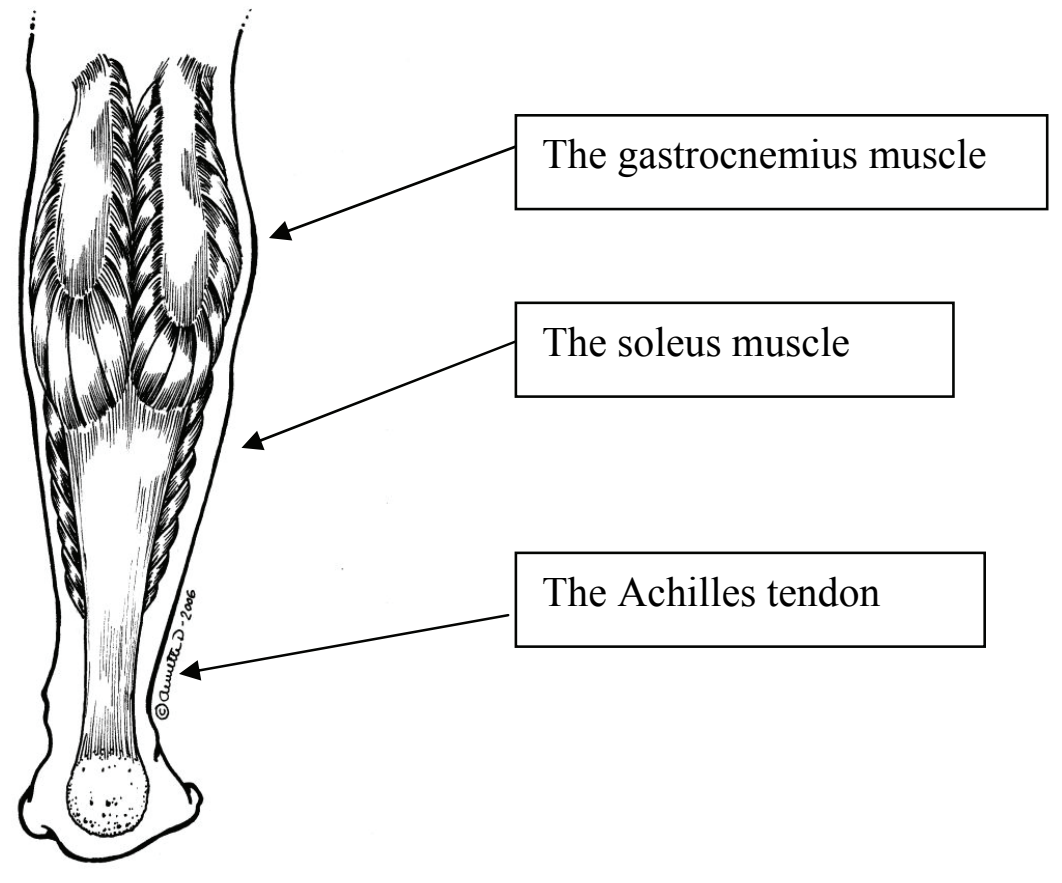

Figure 1. The posterior aspect of the lower leg

\section{Tendon structure}

The extracellular tendon matrix of the Achilles tendon is composed of collagen fibers, elastic fibers (elastin), the ground substance and the anorganic components (Kannus, 2000). The collagen accounts for approximately 65$80 \%$ of the dry weight and the elastin for about 1-2\% (O'Brien, 1997, Kannus, 2000). The Achilles tendon is mostly composed of type I collagen, but, in injured tendons, a higher percentage of type III collagen has been found compared with healthy tendons (Leadbetter, 1992, O'Brien, 1997, Maffulli et al., 2000). The collagen provides the tendon with strength to withstand high loads (O'Brien, 1992, Józsa and Kannus, 1997). The elastin is needed to give the tendon its flexibility/elasticity (O'Brien, 1992, Józsa and Kannus, 1997). The ground substance consists of approximately $60-80 \%$ water, proteoglycans and glycoproteins (O'Brien, 1997). The tendon cells (the tenoblasts and tenocytes) are situated in rows between the collagen fibers (Kannus, 2000, Paavola et al., 2002a).

The basic unit of the collagenous structure is the tropocollagen molecule (Józsa and Kannus, 1997, Kannus, 2000, Stanish et al., 2000). It is suggested that five tropocollagen units join to form the microfibril (Stanish et al., 2000). The microfibrils combine in groups which in turn form the collagen fibrils, which are suggested to be the basic load-bearing units of ligaments or 
tendons (Stanish et al., 2000). Groups of collagen fibrils and their surrounding matrix form a collagen fiber and this is the smallest unit visible using light microscopy (Kannus, 2000). A fiber may be as long as the tendon itself. The endotenon is a fine sheath of connective tissue which surrounds the collagen fibers and binds them together (Kannus, 2000). A primary fiber bundle (subfascicle) is formed by a bunch of fibers. A group of secondary fiber bundles (fascicle), which is formed by a group of primary fiber bundles, form the tertiary bundle. Nerves and blood vessels enter the tendon along the endotenon. Groups of tertiary bundles are surrounded by the epitenon and form the actual tendon (Kannus, 2000). Outside the epitenon, there is an outer sheath, the paratenon, and between these two layers there are fluids which provide lubrication, prevent friction and protect the tendon (Józsa and Kannus, 1997, Kirkendall and Garrett, 1997, Kannus, 2000). It is important to remember that the classification and nomenclature of the tendon structure can vary in the literature and there is no set standard.

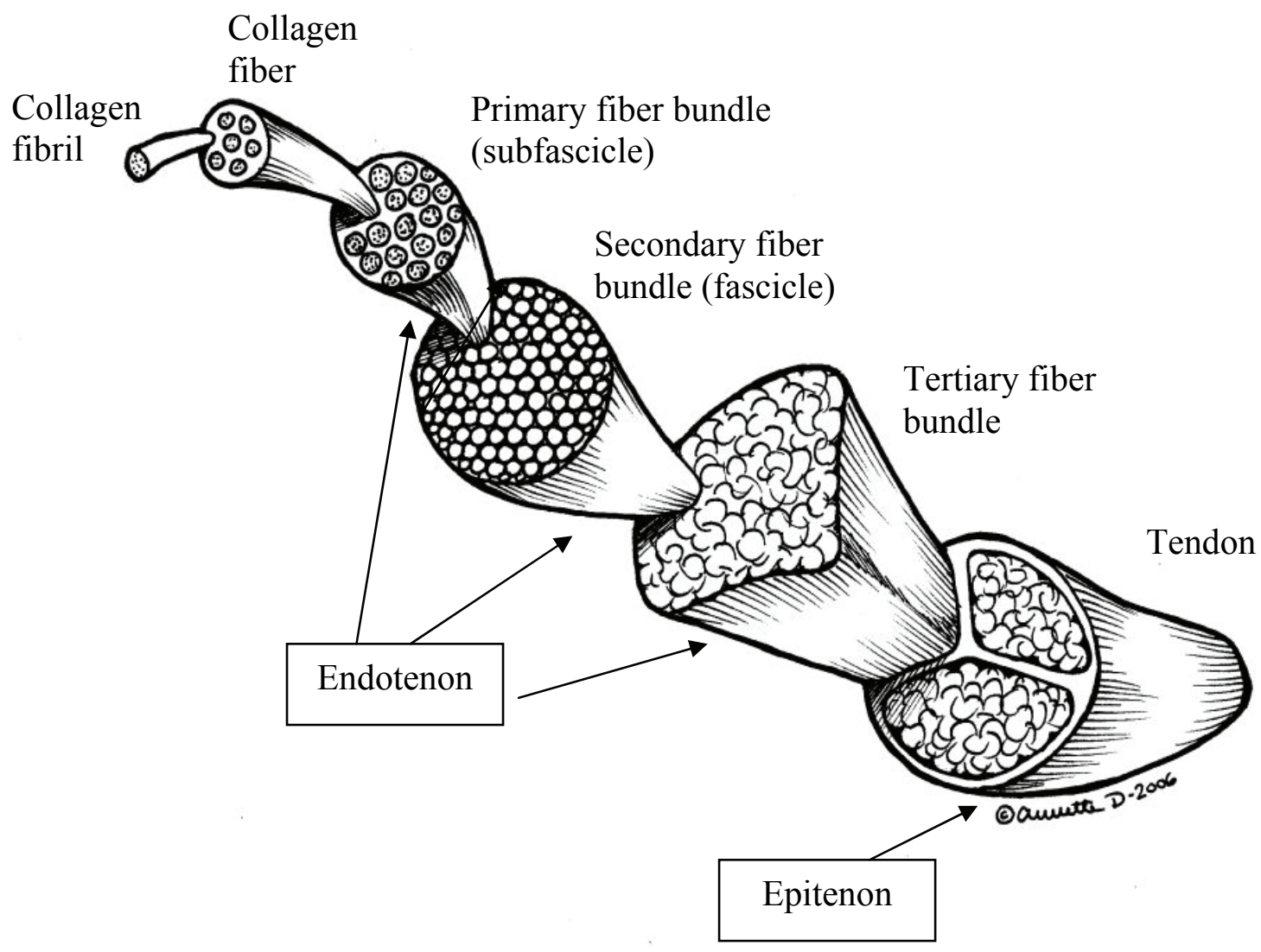

Figure 2. Schematic image of the tendon structure 


\section{Circulation}

The Achilles tendon receives its blood supply from three regions; the myotendinous junctions, osteotendinous junctions and the paratenon (Schatzker and Brånemark, 1969, Carr and Norris, 1989, O'Brien, 1997). It has been reported that the midportion of the Achilles tendon has a lower vascular supply compared with the rest of the tendon (Lagergren and Lindholm, 1959, Carr and Norris, 1989). More recent studies have reported contradictory results. Åström and Westlin (1994b) found even blood distribution in the Achilles tendon, with a decrease seen at the insertion. It has also been shown that injured Achilles tendons have an increased blood flow in the symptomatic area compared with controls (Åström and Westlin, 1994a, Öhberg et al., 2001, Knobloch et al., 2006). Despite differences in findings regarding the blood flow to the Achilles tendon, there appears to be a consensus that there are reductions in the blood flow with increasing age (Tuite et al., 1997, Langberg et al., 2001a).

One important clinical finding is that exercise, both isometric and dynamic, causes increased circulation in the peritendinous region of the Achilles tendon (Langberg et al., 1998, Langberg et al., 1999a). Langberg and co-workers (2001a) have shown that exercise increases the circulation in the tendon by 2.5-3.5 times the resting rates in subjects, independent of age. This supports the concept of giving patients exercise to promote circulation in order to help the healing process in all phases of rehabilitation. The research performed on blood flow indicates that tendon tissue is very much a dynamic tissue which responds to muscular activity (Kjaer et al., 2000).

\section{Innervation}

The Achilles tendon is innervated by nerves from the surrounding muscles and by small fasciculi from cutaneous nerves, particularly the sural nerve (Stilwell, 1957). As it is a very large tendon, the Achilles tendon has a relatively small number of nerve fibers and nerve endings compared with the smaller tendons involved in fine movements (Józsa and Kannus, 1997).

There are four types of nerve ending in the tendon (Józsa and Kannus, 1997, O'Brien, 1997). Three are mechanoreceptors (types I-III) that convert physical energy to afferent nervous signals. They are found both inside and on the surface of the tendon. The type I endings, also called Ruffini corpuscles, are pressure sensors and have a relatively low threshold in reaction to pressure (Józsa and Kannus, 1997, O'Brien, 1997). They are sensitive to stretching, adapt slowly and are involved in sensing the stresses within the tendon. The type II endings, also called Vater-Pacini corpuscles, are also pressure sensors 
but adapt relatively quickly and therefore primarily react to acceleration and deceleration (Józsa and Kannus, 1997, O'Brien, 1997). They are involved in the change of movement. The type III endings, also called the Golgi tendon organs, are tension receptors and signal position and react to both active contraction and passive stretch (Józsa and Kannus, 1997, O'Brien, 1997). The fourth type of nerve ending is the free nerve endings that function as pain receptors and are abundant in peritendinous tissues (Józsa and Kannus, 1997).

\section{Metabolism}

Historically, it was thought that tendon tissue was metabolically inert but today it is well known that tendon cells have an active metabolism (Vailas et al., 1978). The oxygen consumption of the Achilles tendon has, however, been shown to be 7.5 times lower than that in skeletal muscle as measured in the rat (Vailas et al., 1978). Even though tendon has a lower metabolic rate when compared with muscle tissue, it is apparently adequate for the needs of the tendon tissue (Józsa and Kannus, 1997). In healthy tendons there is a balance between collagen synthesis and degradation (O'Brien, 1997). The tendon cells synthesize collagen, elastin and proteoglycans and structural glycoproteins (Józsa and Kannus, 1997). The synthetic activity is high during growth and diminishes with age (Józsa and Kannus, 1997). The important clinical aspect of the metabolic rate of the tendon is its relatively slow response to exercise and relatively slow rate of recovery after injury compared with muscle. This has to be taken into account during rehabilitation after injury and surgery. The maturation and remodeling phase of tendon healing is reported to occur from 3 weeks to 12 months after tendon rupture (Enwemeka, 1989b, Leadbetter, 1992, Józsa and Kannus, 1997, Kannus et al., 1997c, Kader et al., 2002). A patient with an injury to the Achilles tendon must therefore expect a recovery time of 3 months to a year, and sometimes longer, depending on the severity of the injury.

\section{Biomechanics of tendon tissue}

Tendons are designed to transmit high forces from muscle to bone. The physical characteristics of tendon provide for good tensile strength with optimal elasticity with tensile-stretch forces. Tendon is, however, less able to withstand shear and compression forces (Giffin and Stanish, 1993, Józsa and Kannus, 1997, Stanish et al., 2000). The tendon fibers align themselves in the direction of the forces along the tendon (Józsa and Kannus, 1997). A stressstrain curve is often presented in the literature when describing the mechanical behavior of tendon (Figure 3) (O'Brien, 1992, Józsa and Kannus, 
1997, Stanish et al., 2000). At rest, the tendon fibers display a wavy configuration. This disappears when the tendon is stretched approximately $2 \%$. When the force is released, the tendon fibers resume their wavy appearance. This part of the stress-strain curve is called the toe region. After the toe region, the tendon shows a relative linear response to stress. Up to approximately $4 \%$ elongation, the tendon will return to its original state after the tension is released. If the tendon is stressed beyond $4 \%$ of its length, partial ruptures will occur and, at approximately $8 \%$ of elongation, a complete rupture will occur.

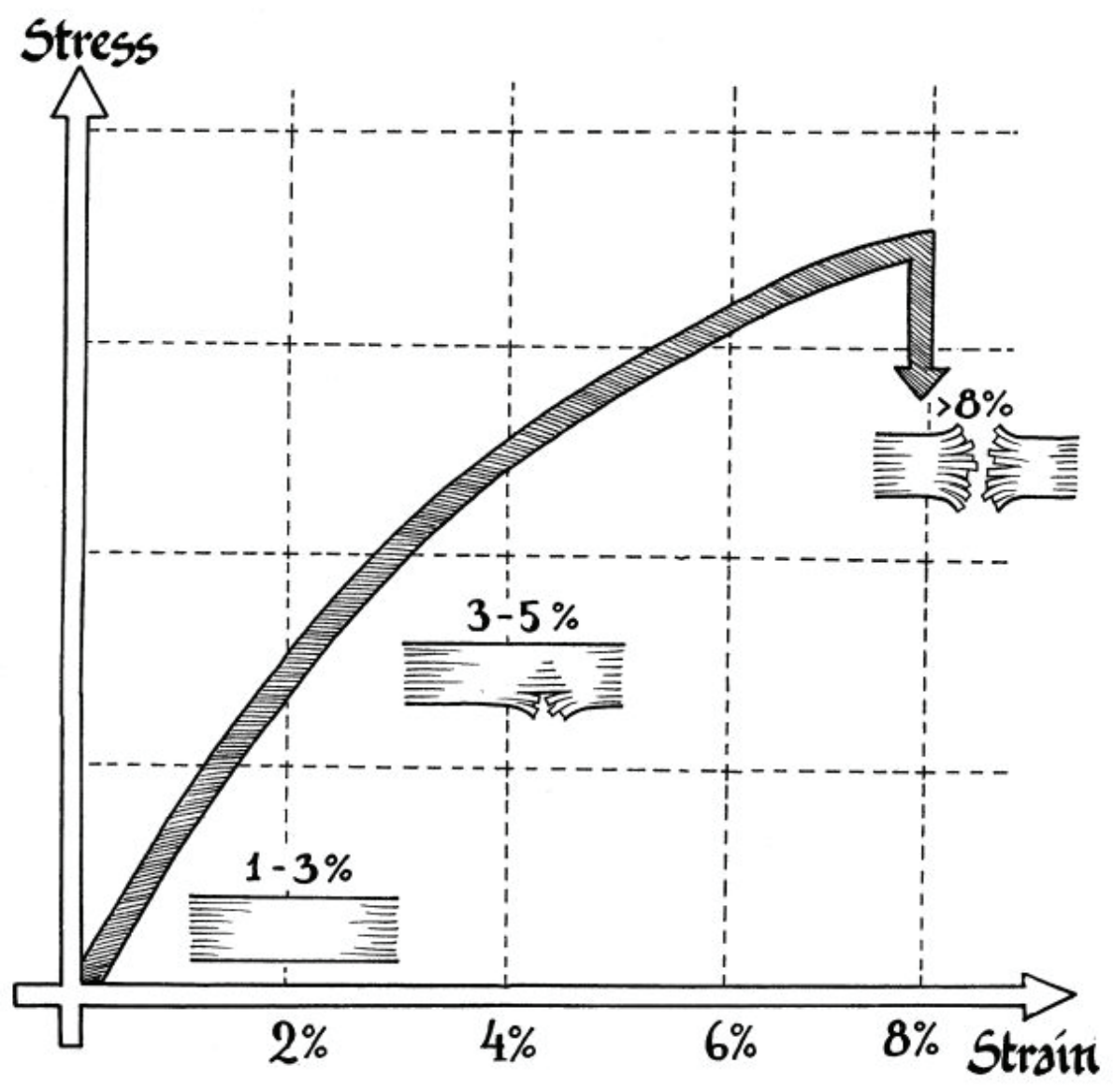

Figure 3. A stress-strain curve of the tendon

The greater the cross-sectional area of a tendon, the larger its capacity to withstand heavy loads before failure and longer tendons have a greater capacity to elongate before failure compared with shorter tendons (Stanish et al., 2000). This means that a tendon that is $2 \mathrm{~cm}$ long will rupture when stretched to a length of $2.16 \mathrm{~cm}$ and that a tendon that is $10 \mathrm{~cm}$ will rupture first when stretched to a length of $10.8 \mathrm{~cm}$. At low rates of loading, tendons 
are more viscous or ductile. Tendons therefore absorb more energy at low rates of loading compared with high ones. At high rates of loading, the tendons become more brittle and absorb less energy, but they are then more effective in moving high loads (Józsa and Kannus, 1997). The tendon therefore reacts differently to slow stretch compared with jumping activity. The tensile strength of healthy tendons increases during childhood and adolescence and reaches a peak between 25 to 35 years of age, after which the tensile strength slowly decreases (Ippolito 1986 as cited by (Józsa and Kannus, 1997).

In-vivo measurements of loading the Achilles tendon, during various types of activity, were performed with a buckle type of transducer by Komi and coworkers (1992). They showed that the forces in the Achilles tendon varied considerably between individuals and that the forces were well above the range of the single load ultimate tensile strength of the tendon. Komi and coworkers (1992) found that, during gait, the Achilles tendon force was built up before heel contact, with a sudden momentary release of the force at early impact. Thereafter, the force in the Achilles tendon was built up relatively quickly during the stance phase, with the peak force measured at push-off. They measured a force of $2.6 \mathrm{kN}$ during walking, while during running the force was $9 \mathrm{kN}$, corresponding to approximately 12 times the body weight. Cycling only produced a force of less than $1 \mathrm{kN}$. They also noticed that the release of force at impact was absent with ball contact running but was present with heel contact running. It has therefore been speculated that ball contact running is a risk factor for Achilles tendinopathy in runners, but there are no scientific data to support this.

A high load on the Achilles tendon occurs in activities during which the so-called stretch-shortening cycle (SSC) is utilized (Komi et al., 1992, Fukashiro et al., 1995b). The SSC is a combination of an eccentric muscle action (with lengthening of the muscle and tendon), followed immediately by a concentric muscle action (shortening of the muscle-tendon complex) (Bosco et al., 1982b, Komi, 2000, Ishikawa and Komi, 2004).

The concentric force production will be higher when it is preceded by an eccentric muscle action compared with a pure concentric muscle action, due to the utilization of the passive elastic components such as the tendon (Figure 4) (Bosco et al., 1982b, Komi, 2000). In Figure 4, the peak concentric power measured during a pure concentric toe raise, with an external load of $23 \mathrm{~kg}$, was $690 \mathrm{~W}$. When the concentric contraction was preceded by an eccentric contraction, the peak concentric power measured was, however, $1283 \mathrm{~W}$. 


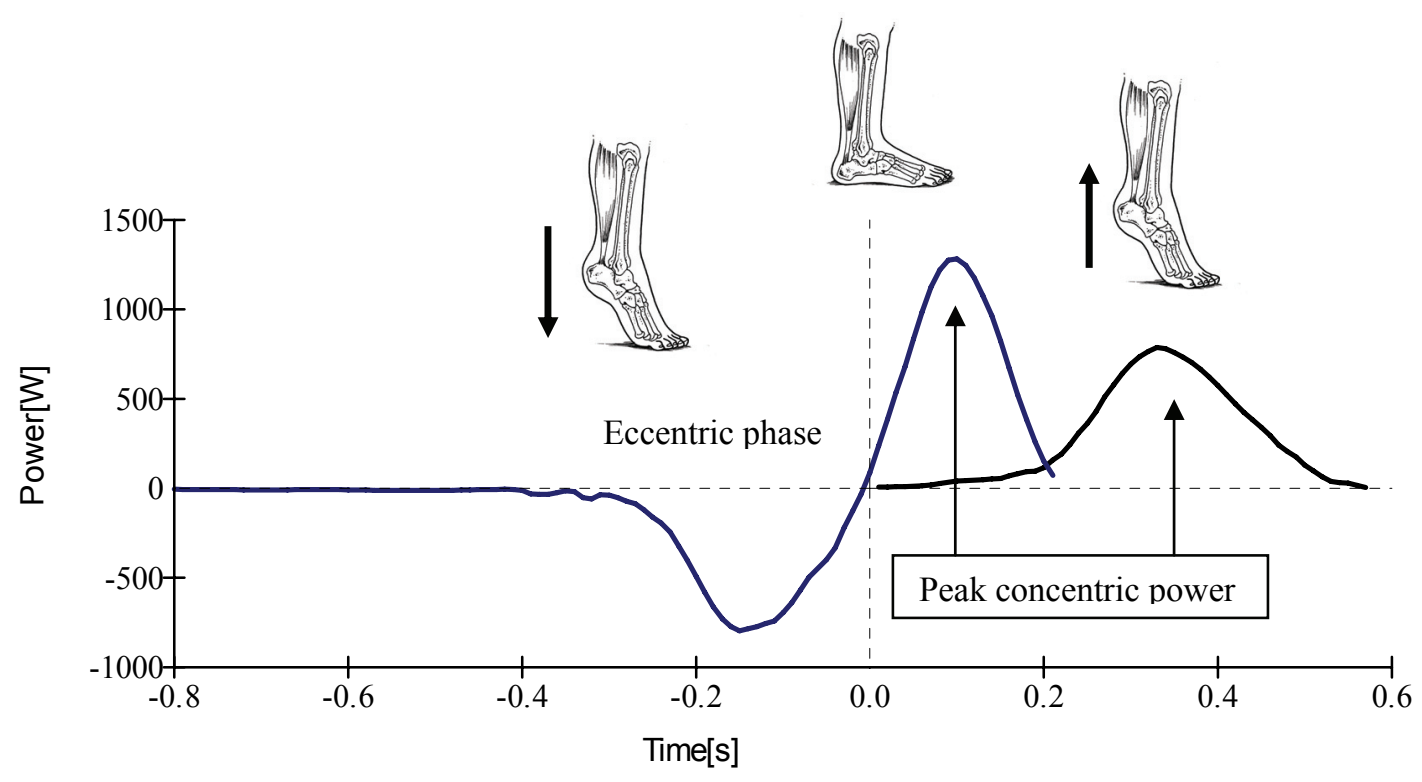

Figure 4. Concentric force production (in watts) of the calf musculature with and without a preceding eccentric contraction

Komi and co-workers (1992) used three types of jump when measuring Achilles tendon forces. The countermovement jump (CMJ) allows the person to start in an erect position, quickly bend down and then perform a maximum vertical jump. The squat jump (SJ) is a maximum vertical jump starting in a squatting position to eliminate the countermovement. Hopping, which is a submaximum jump, like jumping rope, is characterized by large mechanical work at the ankle with minimal work at the knee and hip joint. The forces measured in the Achilles tendon were $2.2 \mathrm{kN}$ (approximately 3 times body weight) with SJ, $1.9 \mathrm{kN}$ (approximately 2.5 times body weight) with CMJ and $4.0 \mathrm{kN}$ (approximately 5 times body weight) with hopping. The efficiency of utilizing the SSC in the various types of jump reportedly ranges from $17 \%$ with CMJ and 23\% with SJ and 34\% with hopping (Belli and Bosco, 1992, Fukashiro et al., 1995b).

The elasticity of the Achilles tendon is important in order to store and release energy during the SSC and thereby improve the economy and performance of motion (Fukashiro et al., 1995b, Komi, 2000). Arndt and coworkers (1998) also showed that non-uniform stress in the Achilles tendon can occur through modifications of individual muscle contributions. It has, however, not established whether this has any causal effect on overuse injuries to the Achilles tendon. 


\section{Effect of exercise on tendons}

The adaptive responses in tendons to exercise are slower than those seen in muscles. Improvements may take a long time but can, nonetheless be considerable over time. Animal studies provide indications that the tendon is positively affected by physical exercise by becoming larger, stronger and more resistant to injury (Józsa and Kannus, 1997, Kannus et al., 1997c, Buchanan and Marsh, 2001). Animal studies have shown increases in the tensile strength, elastic stiffness and total weight of tendons with exercise (Kannus et al., 1997c). Growing animals appear to have a better response to exercise compared with mature animals (Józsa and Kannus, 1997, Kannus et al., 1997c). The difference appears to be that growing animals respond by increasing the size and weight of tendons, whereas the mature animals respond by improving the structure of the tendon with exercise (Kannus et al., 1997c). However, it has been suggested that physical exercise performed in excess is the main pathological stimulus in overuse damage to the tendon (Leadbetter, 1992, Józsa and Kannus, 1997, Kader et al., 2002, Paavola et al., 2002a). The human Achilles tendon not been studied to the same degree, but Magnusson \& Kjaer (2003) found that intensively trained athletes had a greater cross-sectional area of the Achilles tendon compared with controls. Hansen and co-workers (2003) did not, on the other hand, find any increase in the cross-sectional area of the Achilles tendon after 9 months of regular running in previously untrained individuals. A recent study also reports that the Achilles tendon in athletes that are subjected to intermittent high loads (such as volleyball players) and athletes subjected to repetitive loading (such as runners) also has a larger cross-sectional area when compared with controls (Kongsgaard et al., 2005). It is, however, not clear whether this is an effect of training or a natural selection.

Measurements using microdialysis in the human Achilles tendon show an acute effect by both isometric and dynamic exercise, with increased blood flow in the peritendinous region (Langberg et al., 1999a, Boushel et al., 2000). A study of collagen turnover, measured using microdialysis in the peritendinous regions of the Achilles tendon, showed that an acute bout of exercise increased the collagen synthesis of type I collagen 72 hours after exercise (Langberg et al., 1999b). Further studies by the same researchers of the synthesis and degradation of collagen after a marathon run show that the synthesis of collagen reaches a peak three days after the run and returns to normal levels again after five days (Langberg et al., 2000). The long-term effects of heavy daily exercise (2-4 hours per day) showed an increase in collagen synthesis at week 4, with continued elevated levels at week 11 
(Langberg et al., 2001b). Collagen degradation, on the other hand, was also elevated at week 4 but had returned to a normal level at week 11. The net positive effect on the tendon of this type of physical activity might thus not appear until later, i.e. after 11 weeks. This is in agreement with the clinical experience that numerous patients with Achilles tendon injuries do not have noticeable effects from rehabilitation exercises with changes in symptoms and function until after 3 months of rehabilitation. It is still unclear whether improvements from daily exercise are due to cumulative acute effects or chronic effects. A study by Buchanan and Marsh (2001) of guinea fowl found that treadmill endurance training changed the mechanical properties of the tendon but did not cause tendon hypertrophy. They suggested that the tendon responds to repeated stress by improving its capacity to withstand mechanical fatigue, but that this does not enable the tendon to withstand higher loads (Buchanan and Marsh, 2001). Studies of the effect of strengthening exercise on tendon are almost non-existent, apart from a study by Simonsen and coworkers (1995), who found no effect from strength training on tendon in rats. Strength training has, however, been shown to have positive effect on injuries to the Achilles tendon (Niesen-Vertommen et al., 1992, Alfredson et al., 1998b, Mafi et al., 2001, Silbernagel et al., 2001, Alfredson et al., 2001a, Roos et al., 2004). Endurance type exercise might, however, not have the same effect as strengthening exercise on tendon. Kjaer (2004) suggests that a load of a certain magnitude, together with stretching of the tendon promotes the increased reorganization and synthesis of collagen. The clinical implication when treating runners with Achilles tendon injuries may be that the running needs to be complemented by strength training.

\section{Effect of immobilization on tendons}

Research indicates that immobilization has the opposite effect from exercise/loading (Józsa and Kannus, 1997, Kannus et al., 1997c). Immobilization causes the atrophy of tendon, collagen fibers become thinner and more disoriented and there is a negative effect on the quality of the tendon structure (Kannus et al., 1997c). Immobilization also has negative effects on the non-collagenous tendon matrix. The tendon needs to have force input in order to maintain a structure in which the fibers align in the force direction.

Tendons have a good ability to adapt to loading stimuli. As described by Kannus (1997c), "concerning training and physical activity, the lower the initial loading state, the faster and better the adaptation, and vice versa. 
Concerning disuse and immobilization, the higher the initial loading state, the faster and more severe the atrophy and vice versa." (Figure 5).

This is clinically important when estimating the effect of treatment where persons with low levels of physical activity might respond more rapidly with changes than an athlete. The immobilization due to injury or illness might have a greater impact on an athlete and care should therefore be taken to avoid re-injury when returning to pre-injury/illness activity.

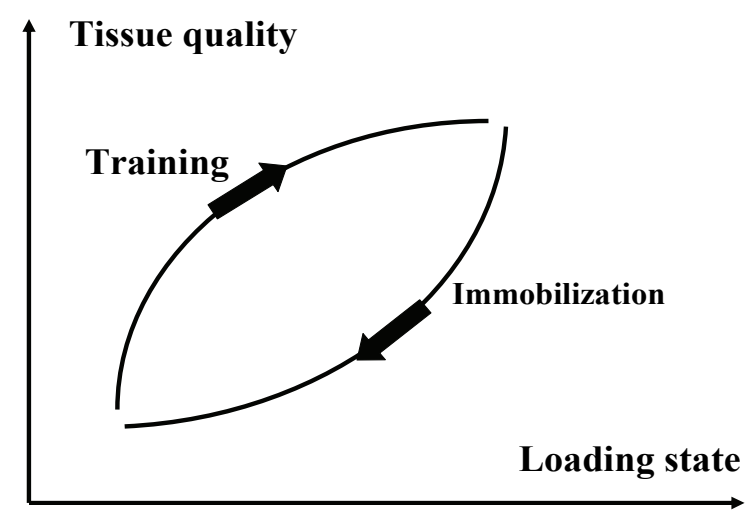

Figure 5. The effect of exercise and immobilization on tendon

\section{Effect of remobilization and reconditioning on tendons}

Remobilization and rehabilitation require more time than is needed to cause immobilization atrophy (Józsa and Kannus, 1997). It is not known whether an injured tendon can ever completely recover to its pre-injury status. It has been found that normal tendons differ from repaired tendons (Maffulli et al., 2000). Normal tendons contain primarily type I collagen, but injured tendons have a higher percentage of type III collagen, which is deficient in the number of cross-links between and within the tropocollagen units (Józsa and Kannus, 1997, Maffulli et al., 2000). Research indicates that the tendon requires mechanical loading in order to recover after immobilization and injury. The optimal amount of loading that is required is, however, still unknown (Enwemeka, 1992, Kjaer, 2004, Ingber, 2005).

\section{Effect of age on tendons}

The tendon is a structure that is subjected to early degenerative changes which start as early as the third decade in life. Aging results in a decline in the structure and function of human tendon (Tuite et al., 1997). The tendon becomes stiffer and loses its elasticity. Taken together, aged tendons are weaker than their younger counterparts and are more likely to tear and suffer from overuse injuries, especially if the tendon also has degenerative 
pathological changes. The highest frequency of tendon ruptures occurs in persons above 30 years of age (Józsa and Kannus, 1997, Möller et al., 2001).

Langberg and co-workers (2001a) evaluated blood circulation in three different age groups (men 26, 48 and 74 years of age) and found a lower resting circulation rate in the oldest age group compared with the other groups. However, all the groups had the same response to exercise, with increases of 2.5-3.5 times the resting level. The literature suggests that wellstructured, long-term exercise will minimize the negative effects attributed to aging (Tuite et al., 1997).

\section{Healing process in tendons}

The healing process following a tendon injury goes through 3 phases (Figure 6) (Enwemeka, 1989b, Leadbetter, 1992, Józsa and Kannus, 1997, Kader et al., 2002). The acute inflammatory phase lasts for up to 3-7 days after injury. During this phase, the inflammatory cells remove the injured tissue, making it possible for phase 2, the proliferative phase, to begin. During the proliferative phase, lasting between 5 and 21 days, new collagen cells are produced. The last phase is the maturation and remodeling phase and it can last for up to a year. During this phase, the tensile strength, elasticity and structure of the tendon are improved.

Kader and co-workers (2002) report that, in animals, the healing tendon regains about $50 \%$ of its tensile strength and $30 \%$ of its energy absorption 15 days after surgery. Thermann and co-workers (2001) reported that ruptured, non-sutured Achilles tendons in rabbits ruptured at $38 \%$ of the force required to rupture the contralateral control side 2 weeks after injury. It has also been reported that a reduction of $30 \%$ in the final biomechanical properties of the tendon can be seen despite the remodeling efforts (Leadbetter, 1992).

\section{Acute inflammatory phase}

2. Proliferative phase

3. Maturation and remodeling phase

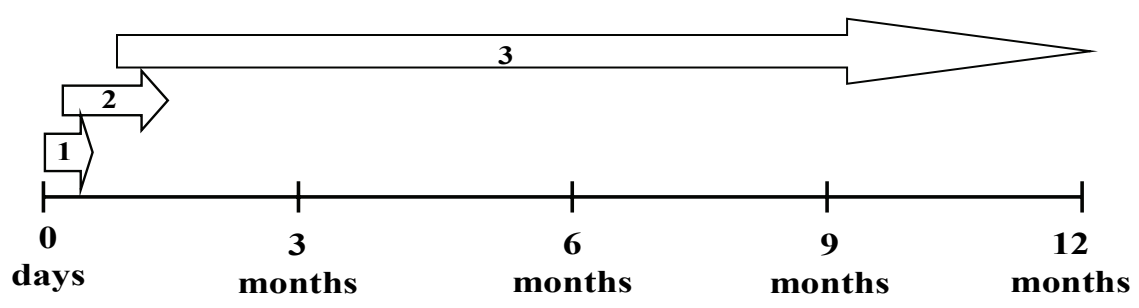

Figure 6. The healing process in tendons 


\section{Achilles tendon injury}

Achilles tendon problems are very common in elite and recreational athletes as well as in the general population. The terminology for describing various injuries to the Achilles tendon varies and is often very confusing. Many terms are used to describe Achilles tendon injury, such as achillodynia, paratendinopathy, paratenonitis, partial ruptures, tendinitis, tendinopathy, tendonitis and tenosynovitis, all of which are used to describe symptoms in the midportion of the tendon. For describing symptoms at the Achilles tendon insertion there are terms such as bursitis, distal achillodynia, enthesitis, insertion tendinopathy, insertion tendonitis, insertitis and retrocalcanear bursitis. Furthermore, there are the terms for ruptured Achilles tendons such as acute total ruptures, spontaneous ruptures or subcutaneous ruptures.

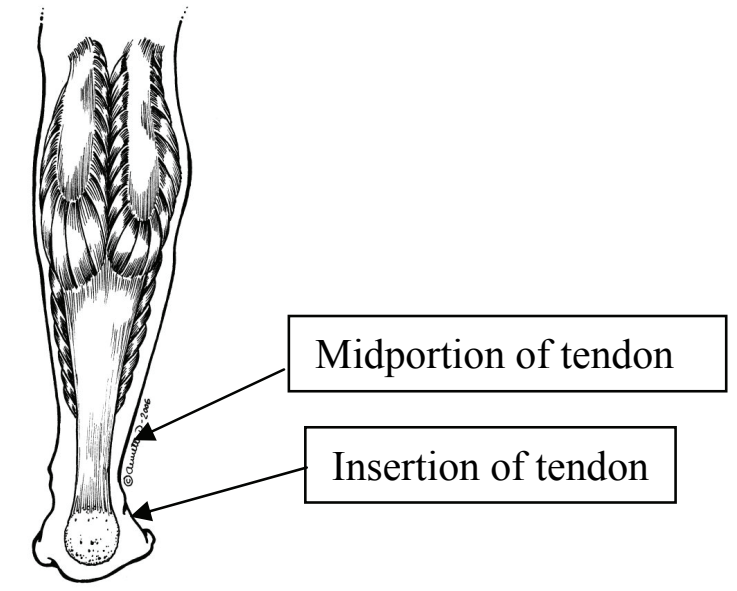

Figure 7. The Achilles tendon

The difficulty with the various terms is when they imply inflammation when none exists or when they are used to describe clinical symptoms when there is no knowledge of the histopathological status of the tissue. Studies of patients with chronic painful Achilles tendon injuries, which are often called tendinitis, have found no signs of inflammation at the site of the injury (Åström and Rausing, 1995, Alfredson et al., 1999). The diagnostic term can then result in the patient receiving the wrong type of treatment, for example. A patient diagnosed with tendinitis (inflammation of the tendon) will be recommended to rest and will be given anti-inflammatory medication. If the diagnosis is instead tendinosis or tendinopathy (non-inflammatory condition), rest would be counterproductive and the patient should start strengthening exercises, while anti-inflammatory medication would have no effect. The habitual use of an injury term can therefore have profound implications in terms of the patient's recovery from injury. 


\section{Classification of Achilles tendon injury}

There are two reasons for the origin of Achilles tendon problems. One is due to overloading the tendon and the other is due to systemic diseases such as rheumatoid arthritis. Only 2\% of Achilles tendon problems are, however, related to systemic disease and the majority of the overloading injuries are related to exercise and sports (Järvinen et al., 2001)

The overloading type of Achilles tendon injuries can be divided into two types, acute and overuse injuries (Figure 8).

\section{Classification of Achilles tendon injury}

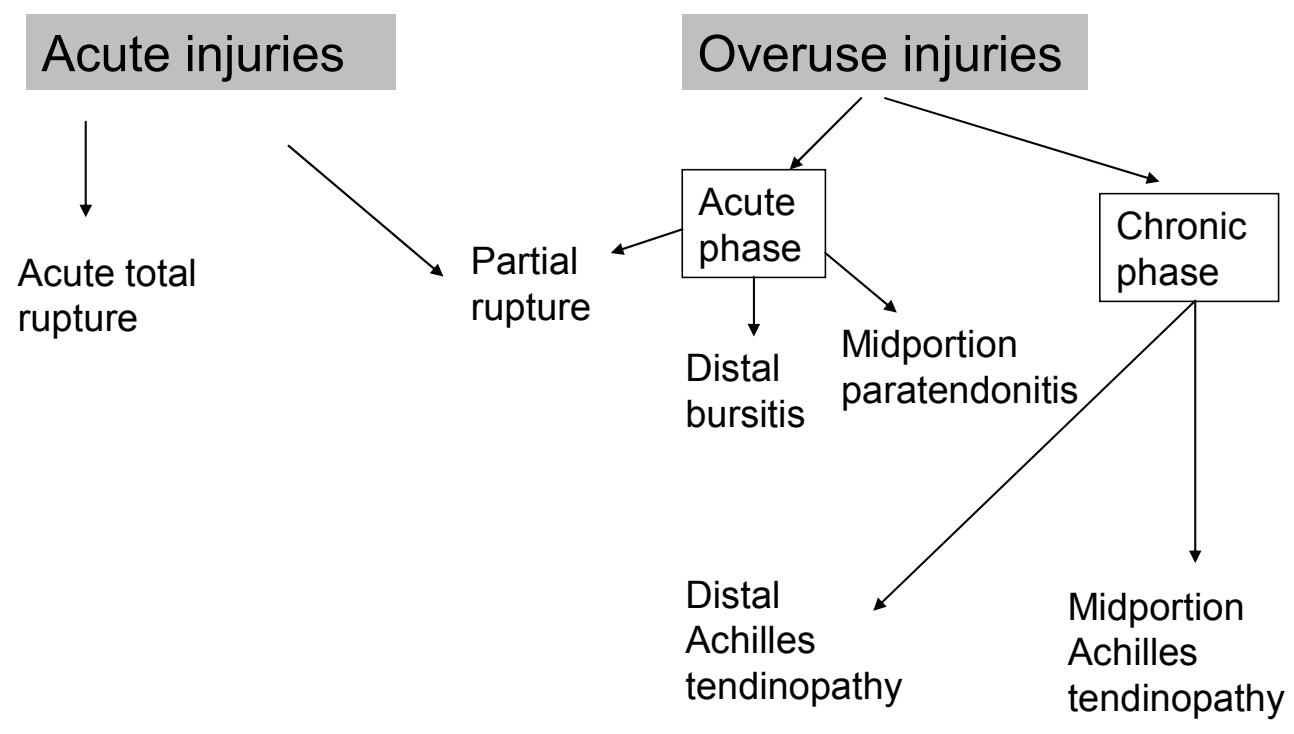

Figure 8. Classification of Achilles tendon injury

1. The acute type of injury is the so-called acute total rupture or partial rupture. These patients have not normally had any symptoms prior to injury. Kannus \& Josza (1991) reported that two thirds of 891 patients with Achilles tendon ruptures had been completely asymptomatic before the rupture.

2. The overuse type of injury to the Achilles tendon is the painful type of injury which is related to repetitive microtrauma causing progressive increase in symptoms as well as difficulty with physical activity. This type of injury may occur when the body's reparative capability is exceeded by repetitive microtrauma (Leadbetter, 1992). The injury can 
be in an acute or chronic phase. The exact time criteria that are used to classify the injury as acute or chronic are arbitrary. In the literature, definitions for the injury to be chronic range from 4 weeks to 3 months, or pain on and off for more than 6 months (el Hawary et al., 1997, Angermann and Hovgaard, 1999, Mafi et al., 2001, Roos et al., 2004). The acute phase injury consists of partial ruptures, bursitis or paratendonitis. The chronic phase injury can be divided into distal Achilles tendinopathy and midportion Achilles tendinopathy depending on the location of the injury.

This thesis concerns the evaluation and treatment of the overuse type of injury in the Achilles tendon. It has been recommended by Maffulli and co-workers (1998) that the clinical syndrome, characterized by a combination of pain, swelling (diffuse or localized) and impaired performance should be called Achilles tendinopathy.

This is also the term used in this thesis. 


\section{Classification of tendinopathies}

Based on the histopathological findings, the classification of injuries to the Achilles tendon can be further divided (Table 1). The various types of injury have then been classified as paratenonitis (inflammation of paratenon), paratenonitis with tendinosis (degenerative changes of tendon), tendinosis and tendinitis (inflammatory response within the tendon) which also includes strains and partial ruptures (Puddu et al., 1976, Józsa and Kannus, 1997, Khan et al., 1999).

Table 1. Bonar's modification of Clancy's classification of tendinopathies (Khan et al., 1999)

\begin{tabular}{|l|l|l|}
\hline $\begin{array}{l}\text { Pathological } \\
\text { diagnosis }\end{array}$ & $\begin{array}{l}\text { Concept } \\
\text { (macroscopic pathology) }\end{array}$ & Histological appearance \\
\hline Tendinosis & $\begin{array}{l}\text { Intratendinous degeneration } \\
\text { (commonly caused by } \\
\text { ageing, microtrauma and } \\
\text { vascular compromise) }\end{array}$ & $\begin{array}{l}\text { Collagen disorientation, } \\
\text { disorganisation and fiber } \\
\text { separation with an increase in } \\
\text { mucoid ground substance, } \\
\text { increased prominence of cells and } \\
\text { vascular space with or without } \\
\text { neovascularisation and focal } \\
\text { necrosis or calcification }\end{array}$ \\
\hline $\begin{array}{l}\text { Tendinitis/ } \\
\text { partial } \\
\text { rupture }\end{array}$ & $\begin{array}{l}\text { Symptomatic degeneration } \\
\text { of the tendon with vascular } \\
\text { disruption and inflammatory } \\
\text { repair response }\end{array}$ & $\begin{array}{l}\text { Degenerative changes as noted } \\
\text { above with superimposed } \\
\text { evidence of tear, including } \\
\text { fibroblastic and myofibroblastic } \\
\text { proliferation, haemorrhage and } \\
\text { organising granulation tissue }\end{array}$ \\
\hline Paratenonitis & $\begin{array}{l}\text { Inflammation of the outer } \\
\text { layer of the tendon } \\
\text { (paratenon) alone, } \\
\text { regardless of whether the } \\
\text { paratenon is lined by } \\
\text { synovium }\end{array}$ & $\begin{array}{l}\text { Mucoid degeneration in the } \\
\text { areolar tissue is seen. A scattered } \\
\text { mild mononuclear infiltrate with } \\
\text { or without focal fibrin deposition } \\
\text { and fibrinous exudate is also seen }\end{array}$ \\
\hline $\begin{array}{l}\text { Paratenonitis } \\
\text { with } \\
\text { tendinosis }\end{array}$ & $\begin{array}{l}\text { Paratenonitis associated } \\
\text { with intratendinous } \\
\text { degeneration }\end{array}$ & $\begin{array}{l}\text { Degenerative changes as noted } \\
\text { for tendinosis with mucoid } \\
\text { degeneration with or without } \\
\text { fibrosis and scattered } \\
\text { inflammatory cells in the } \\
\text { paratenon alveolar tissue }\end{array}$ \\
\hline
\end{tabular}




\section{Achilles tendinopathy - the clinical syndrome, characterized}

by a combination of pain, swelling (diffuse or localized), and impaired performance

Epidemiology - deals with the incidence, distribution, and control of disease in a population

Achilles tendinopathy is associated with activities that include running and jumping, even though it also occurs in persons who are not physically active. Several studies report that the incidence of Achilles tendinopathy in runners accounts for 6-18\% of all injuries (James et al., 1978, Clement et al., 1984, Lysholm and Wiklander, 1987, Soma and Mandelbaum, 1994, Józsa and Kannus, 1997). The highest incidence is usually reported to occur in middleaged individuals, but the exact age to which age this refers is usually not documented (Kvist, 1994, Paavola et al., 2000a, Silbernagel et al., 2001, Paavola et al., 2002a, Alfredson, 2003c). When studying Achilles tendon injuries in athletes, Kvist (1991) found that the mean age of subjects with Achilles tendon disorders was $28 \pm 9$ years, while it was $20 \pm 8$ years in those without these disorders. Treatment studies (both surgical and non-surgical), that not only include athletes, report that the average age of patients with Achilles tendinopathy ranges between 30 and 55 years, with a total range from 17-80 years of age (Nelen et al., 1989, Leach et al., 1992, NiesenVertommen et al., 1992, Schepsis et al., 1994, Alfredson et al., 1998b, Alfredson et al., 1998c, Angermann and Hovgaard, 1999, Paavola et al., 2000a, Paavola et al., 2000b, Mafi et al., 2001, Öhberg and Alfredson, 2002, Paavola et al., 2002b, Neeter et al., 2003, Alfredson and Öhberg, 2005d). In a prospective study, Johansson (1986) reported that the annual incidence of Achilles tendon overuse injury in elite orienteers was $7 \%$. However, the subjects in this study were young athletes (aged $17.5 \pm 1.5$ years) and, since the majority of the Achilles tendon injuries are seen in older individuals, the representativness of the sample can be questioned. The annual incidence may be higher in older runners. In a prospective study of 69 young people (aged $18.41 \pm 1.29$ years) entering a six-week military training course it was found that $14.5 \%$ sustained an Achilles tendon overuse injury (Mahieu et al., 2005). The incidence of Achilles tendinopathy in 449 young men (mean age 22.5 years) entering special naval warfare training was $5.1 \%$ (Kaufman et al., 1999). In an 11-year follow-up of 269 male orienteering runners, with a mean age of 48.6 years, Kujala and co-workers (1999) found that $29 \%$ reported Achilles tendon overuse injury compared with $4 \%$ of the controls. 
Achilles tendinopathy is also reported to occur primarily in men. One study reports that $89 \%$ of the runners with Achilles tendinopathy were men (Kvist, 1991). In a review of several treatment studies, the percentage of men was between $45-86 \%$, with the lower percentages seen in the more recent studies (Nelen et al., 1989, Schepsis et al., 1994, Alfredson et al., 1998b, Alfredson et al., 1998c, Angermann and Hovgaard, 1999, Paavola et al., 2000a, Paavola et al., 2000b, Mafi et al., 2001, Öhberg and Alfredson, 2002, Paavola et al., 2002b, Alfredson and Öhberg, 2005d).

Bilateral injury also appears to be common and, in several studies that include patients with bilateral symptoms, the occurrence of bilateral injury is approximately 30\% (Nelen et al., 1989, Kvist, 1991, Paavola et al., 2002b, Öhberg and Alfredson, 2004a). In an 8-year follow-up of patients with Achilles tendinopathy, it was also found that $41 \%$ of the patents developed symptoms in their previously uninjured tendon (Paavola et al., 2000a).

To summarize; overuse injury to the Achilles tendon occurs at all ages, but more frequently in middle-aged (30-55 years old) individuals, and is related to running and jumping with a high risk of bilateral injury.

Etiology - deals with the causes or origins of disease

The etiology of Achilles tendinopathy is considered to be multifactorial, with a combination of intrinsic (relating to the patient) and extrinsic (external to the patient) risk factors. The various predisposing risk factors related to Achilles tendinopathy that are suggested as being important in the literature are listed in Table 2 (Clement et al., 1984, Lysholm and Wiklander, 1987, Hess et al., 1989, Kvist, 1991, Haglund-Åkerlind and Eriksson, 1993, Kvist, 1994, DeMaio et al., 1995, Kannus, 1997a, Alfredson et al., 1998a, Kaufman et al., 1999, McCrory et al., 1999, van der Linden et al., 1999, Järvinen et al., 2001, Kader et al., 2002, Paavola et al., 2002a, Khaliq and Zhanel, 2003, Järvinen et al., 2005, Mahieu et al., 2005, Alfredson and Öhberg, 2005c).

Table 2. Predisposing intrinsic and extrinsic factors related to Achilles tendinopathy

\begin{tabular}{|l|l|}
\hline Intrinsic factors & Extrinsic factors \\
\hline Age & Environmental condition \\
Biomechanical malalignments of the leg/foot & Excessive loading \\
Decreased flexibility & Improper or faulty equipment \\
Gender & (primarily shoes) \\
Leg length discrepancy & Training errors \\
Muscle weakness or imbalance & Side-effects of drug treatment \\
Tendon blood supply & \\
\hline
\end{tabular}




\section{Intrinsic factors}

The Achilles tendon is subjected to loads as high as six to twelve times the weight of the body during running and jumping and repetitive loading is thought to be one of the main pathological stimuli causing Achilles tendinopathy (Komi et al., 1992, Fukashiro et al., 1995b, Kader et al., 2002, Paavola et al., 2002a). Achilles tendinopathy is an overuse injury which, by definition, means that the injury is caused by repetitive strain of the tendon. All changes in lower leg functions, such as muscle-tendon weakness, increased or decreased flexibility or lower leg malalignments, could independently or together affect the running or walking pattern and in turn affect the way the Achilles tendon is loaded and might therefore be etiological factors for the development of Achilles tendinopathy.

Several studies report some kind of malalignment, such as hindfoot inversion, or foot hyperpronation as being related to Achilles tendinopathy (Kvist, 1991, Kaufman et al., 1999, McCrory et al., 1999). Kvist reports that $60 \%$ of patients with chronic Achilles tendon disorder had some kind of predisposing malalingment of the lower extremity (Kvist, 1991, Kvist, 1994). However, Åström (1997) concluded that malalignments were probably not important and that orthotic treatment was not justified for patients with Achilles tendinopathy. Until there are prospective randomized treatment studies of the correction of proposed malalignments, it is difficult to justify corrections to every malalignment found in patients with Achilles tendon problems.

Decreased ankle dorsiflexion is also reported to be related to an increased risk of Achilles tendinopathy (Haglund-Åkerlind and Eriksson, 1993, Kaufman et al., 1999). On the other hand, a recent study argued that an increase in ankle dorsiflexion range of motion is a risk factor for Achilles tendinopathy (Mahieu et al., 2005). A treatment study that used prolonged stretching into dorsiflexion with a night splint compared with exercise reported that exercise alone produced the better result (Roos et al., 2004).

Decreased ankle plantarflexion strength has been found to be a predictor of Achilles tendinopathy and decreased strength has also been found in injured subjects compared to uninjured subjects (Haglund-Åkerlind and Eriksson, 1993, Alfredson et al., 1998b, Alfredson et al., 1998c, Mahieu et al., 2005).

As described earlier, aging appears to have a negative effect on the mechanical properties and blood supply of the Achilles tendon and might explain why Achilles tendinopathy is most common in middle-aged individuals (Tuite et al., 1997, Langberg et al., 2001a). 
The degree to which these factors influence the occurrence of Achilles tendinopathy, how they interact and their clinical relevance is not yet fully understood. There is therefore a need for more prospective studies of the risk factors for Achilles tendinopathy, especially in older athletes. There is also a need to evaluate the suggested predisposing factors before and after treatment interventions to determine their influence on the injury.

\section{Extrinsic factors}

Predisposing extrinsic factors are thought to be training errors, changes in training surface and ineffective or improper footwear, as well as the sideeffects of drug treatment such as fluoroquinolones (Clement et al., 1984, Hess et al., 1989, Nichols, 1989, Galloway et al., 1992, Kvist, 1994, DeMaio et al., 1995, Kannus, 1997a, van der Linden et al., 1999, Järvinen et al., 2001, Kader et al., 2002, Paavola et al., 2002a, Khaliq and Zhanel, 2003, Järvinen et al., 2005).

The excessive loading of the Achilles tendon and training errors such as increasing intensity and duration too quickly are reported to be present in 60$80 \%$ of patients with Achilles tendinopathy (Kvist, 1991, Kvist, 1994, Järvinen et al., 2001, Järvinen et al., 2005). Some studies have found a relationship between years of running and incidence of Achilles tendinopathy (Haglund-Åkerlind and Eriksson, 1993, McCrory et al., 1999). HaglundÅkerlind and Eriksson (1993) also reported that injured runners had not only run for more years but had also covered longer distances.

The use of fluoroquinolone antibiotics (normally used in respiratory infections) has been implicated in tendinopathy and tendon ruptures (van der Linden et al., 1999, Khaliq and Zhanel, 2003). In a literature review, Khaliq and Zhanel (2003) found the incidence of Achilles tendinopathy in a healthy population using fluoroquinolone to be low, but increases were found in patients who had renal dysfunction, were undergoing hemodialysis, or had received renal transplants. They also report that the median duration of fluoroquinolone treatment before onset was 8 days, although it could start as early as 2 hours after the first dose and as late as 6 months after treatment.

There is, however, no substantial scientific evidence relating to how and how much most of these extrinsic factors predispose subjects to the occurrence of Achilles tendon disorders. There is also a lack of prospective studies of extrinsic risk factors in Achilles tendinopathy. 
Pathogenesis - the development of a diseased or morbid condition

The overuse type of injury to the Achilles tendon is the painful type of injury that is related to repetitive microtrauma which causes a progressive increase in symptoms as well as difficulty with physical activity. This type of injury occurs when the body's reparative capability is exceeded by repetitive microtrauma (Figure 9) (Leadbetter, 1992). Repetitive activity which strains the tendon to $4-8 \%$ of its original length leads to cumulative microtrauma and is thought to start the overuse injury process in the Achilles tendon (Józsa and Kannus, 1997, Kannus, 1997a). The repetitive overloading may weaken the collagen cross-linking, noncollagenous matrix and vascular elements of the tendon (Józsa and Kannus, 1997). The reparative ability of the tendon tissues is exceeded by the repetitive destructive loading. Even strong materials can fatigue after repeated submaximum loads.

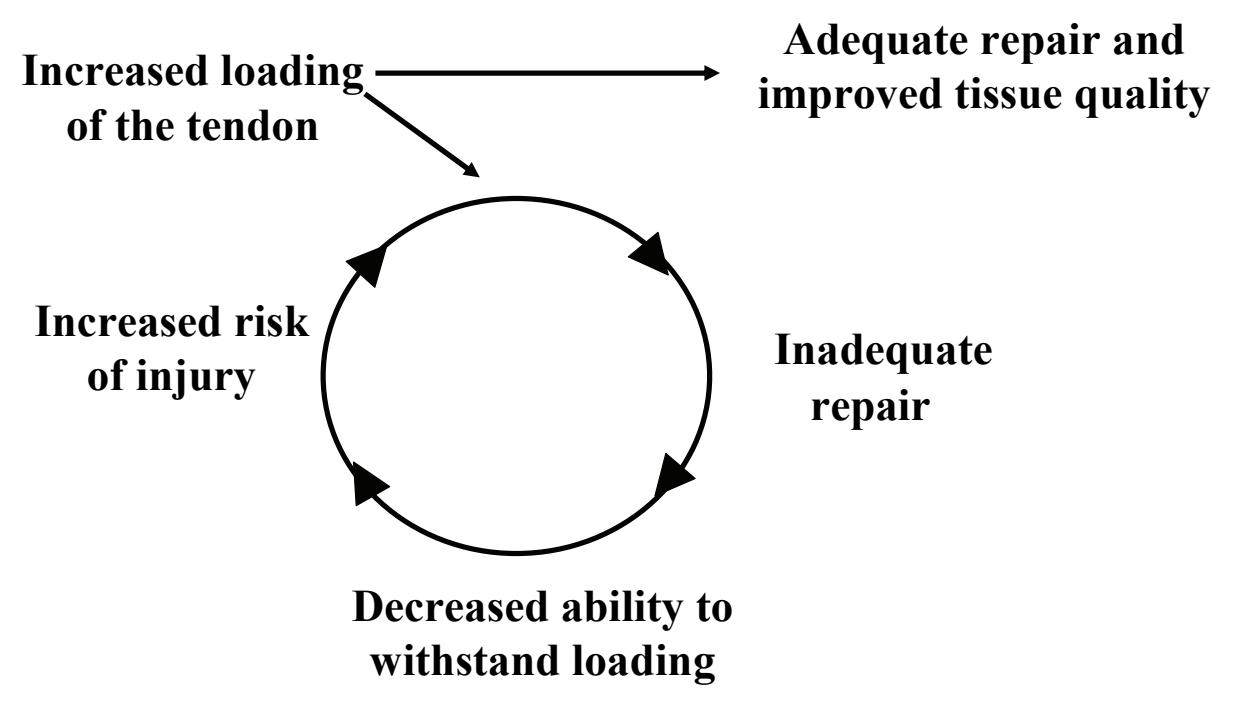

Figure 9. The overuse type of tendon injury

It has been suggested that a reduced vascular supply to the midportion of the Achilles tendon is related to the pathogenesis of Achilles tendinopathy (Lagergren and Lindholm, 1959, Carr and Norris, 1989, Kannus, 1997a, Alfredson et al., 2003b). It has also been suggested that reduced blood circulation causes impaired metabolic activity and disturbed oxygen transport and is therefore detrimental to the tissue repair (Tuite et al., 1997, Kannus, 1997a). This is, however, controversial and several research reports suggest that there is no reduction in blood supply to the midportion of the tendon (Åström and Westlin, 1994a, Öhberg et al., 2001, Knobloch et al., 2006).

It is known that, during running and jumping, the Achilles tendon is subjected to loads as high as six to twelve times the weight of the body and 
this high repetitive loading is thought to be one of the main pathological stimuli causing Achilles tendinopathy (Komi et al., 1992, Fukashiro et al., 1995b, Kader et al., 2002, Paavola et al., 2002a). The high load on the Achilles tendon occurs in activities during which the so-called stretchshortening cycle (SSC) is utilized (Komi et al., 1992, Fukashiro et al., 1995b). If the calf muscle is weak or fatigued, the capacity of the muscle to withstand loads is reduced and its ability to protect the Achilles tendon from injury is therefore diminished. If the Achilles tendon is given adequate time and loading status, it will recover and might be stronger than before. If the recovery time is too short, the cumulative trauma will lead to major injury and possible tendinosis (Figure 9). It is suggested that there is a fine line between adequate and healthy loading and overloading of the Achilles tendon (Figure $10)$.

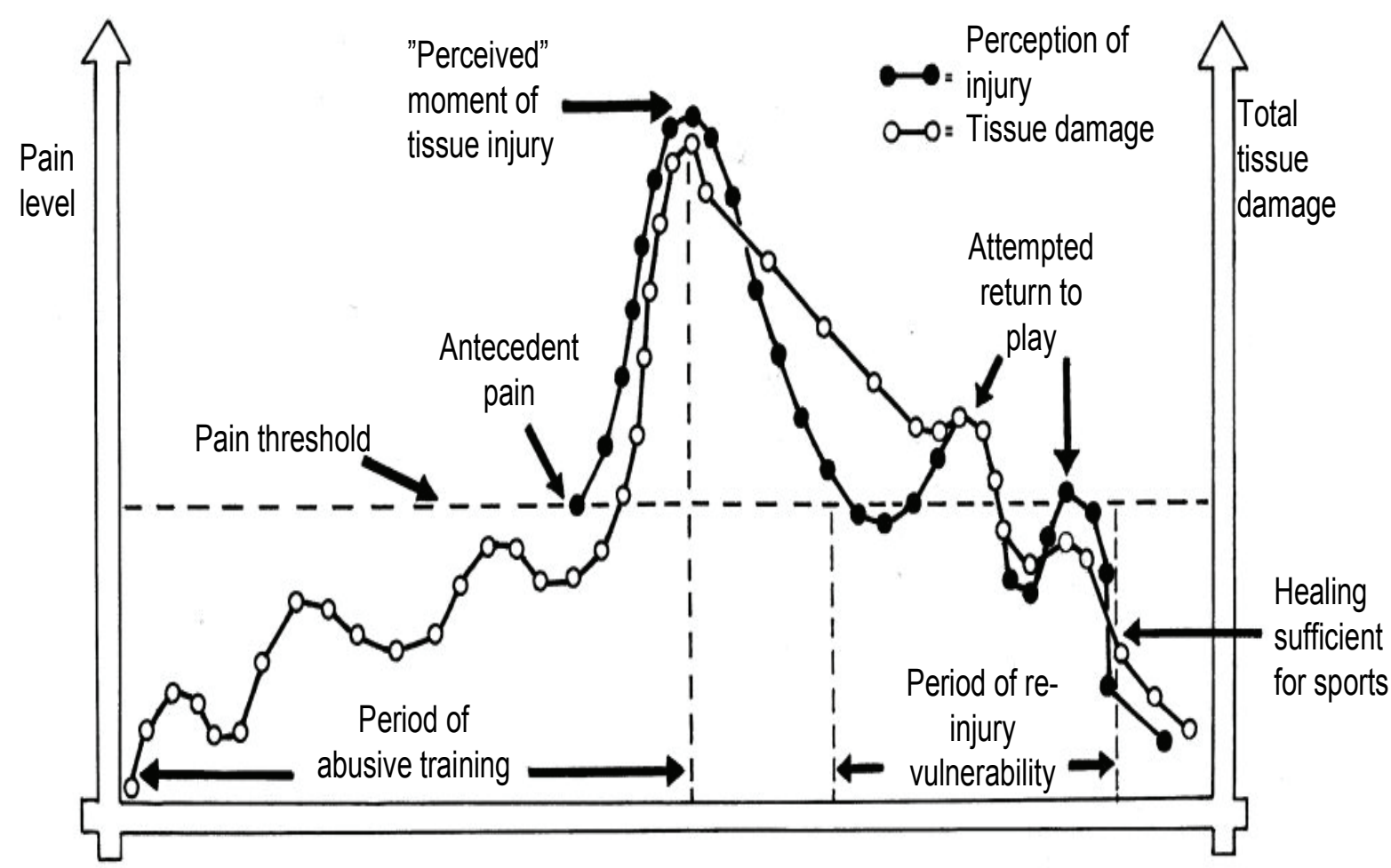

Onset of activity

Months

Figure 10. Schematic illustration of pain and tissue damage in overuse tendinopathy. (reproduced based on Leadbetter (1992), with permission). 
Pathophysiology - the functional changes associated with or resulting from disease or injury

In 163 patients with chronic Achilles tendinopathy, surgical and histopathological findings showed that $90 \%$ had degenerative changes, socalled tendinosis (Åström and Rausing, 1995). Degenerative changes were also found in $20 \%$ of non-symptomatic tendons. It was also found that $19 \%$ of the patients had partial ruptures which always occurred in the tendinosis area. There was also a lack of inflammatory cells and poor healing response in the biopsies. Åström and Rausing (1995) describe the surgical findings in an Achilles tendon with tendinopathy as a loss of the normally glistening white appearance of the tendon; it becomes grey and the tendon thickens.

The degenerative changes in the tendon can be divided into several subcategories such as hypoxic, mucoid, hyaline, lipoid, fibrinoid, calcific or a combination of these (Józsa and Kannus, 1997, Maffulli et al., 2003b). The degenerative changes can be the result of a variety of causes such as aging, microtrauma, vascular compromise, or other reasons and they may vary from tendon to tendon (Józsa and Kannus, 1997). The histopathological findings of tendinosis are collagen disorientation, disorganization and fiber separation (see Table 1 on the classification of tendinopathies). Tendinosis might also occur together with the involvement of the paratenon. This can present itself as crepitation due to adhesions between the tendon and the paratenon.

\section{Differential diagnoses}

There are also other painful conditions in the lower leg which can be mistaken for Achilles tendinopathy and need to be considered when performing the clinical examination. Differential diagnoses of this kind are listed in Table 3.

\section{Table 3. Examples of differential diagnoses of Achilles tendinopathy}

Anomalous soleus muscle

Lower leg compartment syndrome

Os trigonum syndrome

Plantar fasciitis

Posterior tibial stress syndrome

Referred pain due to low back injury

Stress fracture of the ankle or lower leg

Tarsal tunnel syndrome

Tenosynovitis or dislocation of peroneal tendons

Tenosynovitis of the plantar flexors of the foot

Total Achilles tendon rupture

Tumors of the Achilles tendon 


\section{Evaluation}

\section{Clinical assessment}

Achilles tendinopathy is a clinical diagnosis for the clinical syndrome, characterized by a combination of pain, swelling (diffuse or localized), and impaired performance of the Achilles tendon. Clinically, a distinction between midportion (2-6 $\mathrm{cm}$ proximal to tendon insertion) and distal (insertion to the calcaneus) Achilles tendinopathy can be made on the basis of the location of the pain. The symptoms of Achilles tendinopathy are pain during and after physical activity, tenderness on palpation and morning stiffness (Kader et al., 2002, Paavola et al., 2002a, Alfredson, 2003c). With increased severity, patients may also have pain during daily functional activities (Kader et al., 2002, Paavola et al., 2002a).

These patients have often had pain on and off for many years. If they discontinue their activity, the symptoms subside, but, as soon as they resume their physical activity, the symptoms re-occur (Kvist, 1994). The patients also have pain on palpation and sometimes local swelling of the tendon. It is also important to remember that $2 \%$ of Achilles tendon injuries can be due to systemic disease such as rheumatoid arthritis or other inflammatory joint diseases (Järvinen et al., 2001).

\section{Midportion Achilles tendinopathy}

Midportion Achilles tendinopathy is reported to account for $55-65 \%$ of all the Achilles tendon injuries (Kvist, 1991, Järvinen, 1992, Kvist, 1994, Järvinen et al., 2005). The patient history is very important and the onset of symptoms, injury mechanism, possible previous Achilles tendon injury and what makes the symptoms better or worse should be carefully documented.

Patients usually describe a gradual onset of pain. However, they occasionally report a single incident that starts the symptoms. Many patients have had pain for many months or on and off for many years. Initially, the symptoms occur after heavy physical activity, but, as the injury progressess some patients develop pain during physical activity. Sometimes patients also have pain in connection with daily activities, such as walking. Patients describe tendon stiffness in the morning and/or after sitting for longer periods of time. The main complaint is pain with activity, but in some severe cases the patients also report pain at night. If the patients' physical activity has been impaired for a long time, some report muscle-cramp pain in the calf muscle. In the literature, there are reports of a correlation between pain level, morning 
stiffness and severity of disease (Sandmeier and Renström, 1997, Kader et al., 2002, Paavola et al., 2002a, Vora et al., 2005).

Clinically, the patients report pain on palpation in the middle part of the tendon (2-6 cm proximal to the tendon insertion). Sometimes there is a palpable thickening, usually in the more chronic stages. Noticeable crepitation can be indicative of adhesions of the paratenon and paratendinopathy in more acute stages. A thorough physical examination is important in order to rule out any other causes of the pain (see Table 3 for differential diagnoses).

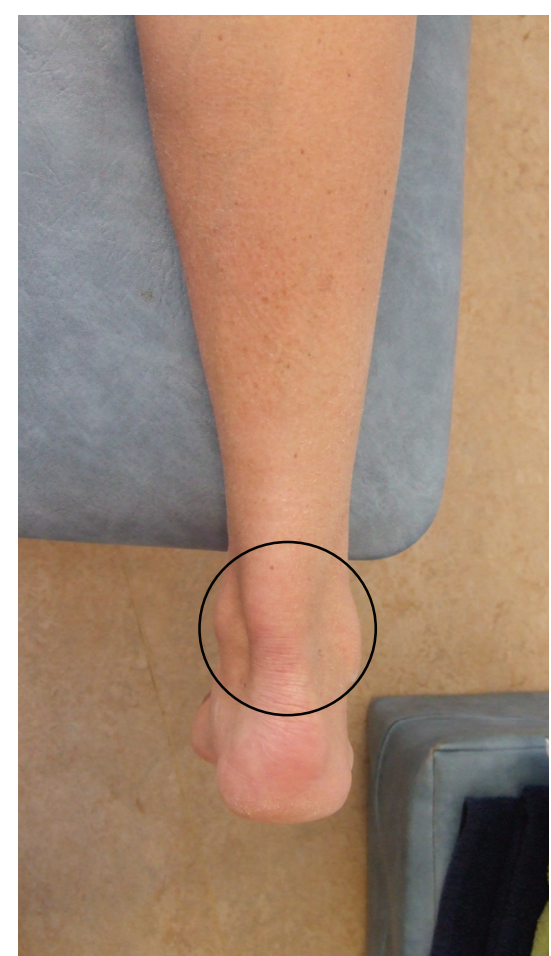

Figure 11. An Achilles tendon with a visible thickening in the midportion

\section{Distal Achilles tendinopathy}

Approximately $20-25 \%$ of all Achilles tendon injuries are reported to be distal and are also called insertional Achilles tendinopathy (Kvist, 1991, Järvinen, 1992, Kvist, 1994, Järvinen et al., 2005) . In a study in 1976-1986 of patients with Achilles tendon injury, $23 \%$ had pain distally and, of these, $61 \%$ were diagnosed as insertiotendinitis, $21 \%$ as retrocalcaneal bursitis and $18 \%$ as both (Kvist, 1991).

These patients report the same complaints as in midportion injury and/or pain related to the type of shoe/athletic wear. In this case the pain can occur due to external compression on the tendon insertion. Swelling around the Achilles tendon insertion to the calcaneus, with redness and warmth, could also be present and might be related to active bursitis. The patients sometimes also report pain after having run uphill, standing on a ladder, or walking 
barefoot on sand. Clinically, there is pain when the tendon insertion is palpated and there is often also pain slightly more medially due to bursitis. This type of injury can also be caused by compression injury of the tendon and the bursa onto the calcaneus, so-called posterior impingement. A prominent superior projection of the calcaneus, i.e. Haglund's deformity, can be the cause of the posterior impingement. For differential diagnoses see Table 3.

\section{Outcome measures}

The evaluation of patients with Achilles tendinopathy described in the literature is mainly based on the patient's report of symptoms with Achilles tendon loading activities. Few methods are used in treatment studies to evaluate the proposed etiological factors such as decreased strength, ROM, biomechanical malalignments and their effects on function. Achilles tendinopathy appears to cause difficulties with physical activity in the active population, but exactly how Achilles tendinopathy affects patients' physical performance is still unclear (Paavola et al., 2002a, Cook and Purdam, 2003).

Tallon and co-workers (2001) have reviewed the outcome after surgery in patients with chronic Achilles tendinopathy and pointed to deficiencies in outcome assessment and outcome criteria. They recommend the use of outcome assessments that are condition specific, sensitive, correlate with clinical severity and are reliable. The various methods used for evaluating patients with Achilles tendon injury are reviewed below.

\section{Physical examination}

An evaluation of standing posture, balance and anatomical malalignments is usually performed during a clinical examination. There are various techniques for evaluating the subtalar varus and forefoot varus as measures of varus alignments that would cause functional hyperpronation during walking and running. Clinically, measurements are often made in non-weight bearing with a goniometer or in weight-bearing describing the foot as cavus, neutral or pronated (James et al., 1978, Clement et al., 1984, Kvist, 1991, Kaufman et al., 1999). A clinical classification of a high, normal and low arch can also be performed, but this is mostly a subjective measure by the evaluator with possible clinical benefits regarding decision-making if there is a need for custom-made orthotics. Motion analysis systems and force plates have also been used to evaluate foot morphology in studies relating to Achilles tendinopathy (Lowdon et al., 1984, Kaufman et al., 1999). 
Palpation of the tendon, the tendon insertion and the documentation of any nodular swelling, crepitation, warmth, redness and level of pain with palpation are clinically relevant. Even though Cook and co-workers (2001) found good reliability for palpation in patients with patellar tendinopathy, they also reported that tenderness on palpation can occur in patients without tendinopathy (Cook et al., 2001).

The literature reports evaluating whether the nodular swelling is in the tendon by palpating the thickening and then having the patient move his/her foot up and down to feel whether the thickening moves (Kader et al., 2002). If it does, this is supposed to be an indication that the nodular thickening is within the tendon.

Measurements of ankle range of motion are usually performed in the clinic, but also made in research studies. The literature reports that Achilles tendinopathy is related to both decreased and increased range of motion. Measurement techniques vary from goniometric evaluations to measurements with passive motion in an hydraulic isokinetic dynamometer (HaglundÅkerlind and Eriksson, 1993, Kaufman et al., 1999, Costa et al., 2005, Mahieu et al., 2005). Goniometric measurements can be performed in the supine or standing position and both passively or actively with care taken to have the foot in a subtalar neutral position. The arms of the goniometer should be aligned, with the proximal arm along the midline of the fibula, the fulcrum by the lateral malleoli and the distal arm parallel to the fifth metatarsal (Norkin and White, 1985). Regular goniometric range of motion evaluations have been shown to be reliable, with intra-tester reliability being higher than inter-tester reliability (Boone et al., 1978, Rothstein et al., 1983). HaglundÅkerlind and Eriksson (1993) used ankle range of motion in an hydraulic isokinetic dynamometer to obtain a more objective measure of muscle flexibility.

Circumference measurements are often used clinically to determine gross muscular atrophy, but they cannot be used to determine muscle quality. Circumference measurements are affected by swelling, and body composition (fat versus muscle) and increases in circumference may not indicate increased muscle mass or vice versa. Techniques include documenting maximum circumference or measuring at predetermined positions relating to bony landmarks (Paavola et al., 2002b). Möller and co-workers (2005) found good reliability for calf circumference measurements (ICC 0.97). 


\section{Outcome scores - questionnaire}

Most research studies have not used specific outcome measures validated for Achilles tendinopathy. Instead, many studies use patient-reported pain (on a Visual Analog Scale) with tendon loading activity as a main outcome measure to determine the success of treatment. Sometimes, it is the investigator that grades the success of treatment based on patient-reported improvements (Clement et al., 1984, Lowdon et al., 1984, Nelen et al., 1989, Leach et al., 1992, Niesen-Vertommen et al., 1992, Schepsis et al., 1994, Alfredson et al., 1998b, Angermann and Hovgaard, 1999, Paavola et al., 2000b, Mafi et al., 2001, Testa et al., 2002, Öhberg and Alfredson, 2002, Alfredson and Lorentzon, 2003a, Gill et al., 2004, Koenig et al., 2004, Paoloni et al., 2004, Öhberg et al., 2004b, Costa et al., 2005, Alfredson and Öhberg, 2005d).

In order to minimize the response bias, which can affect the patients' reports of pain and symptoms, it is important to use outcome assessments in which the investigator has a minimal influence on the scoring (Coleman et al., 2000, Tallon et al., 2001). In many of the above studies, it is difficult to judge how much the investigator could have influenced the results. Many studies include questionnaires relating to symptoms, physical activity, other treatments and previous injuries, but they are rarely specific standardized questionnaires.

A performance test protocol and scoring scale for the evaluation of ankle injuries by Kaikkonen and co-workers (1994) has been used in a follow-up study of patients with Achilles tendinopathy (Kaikkonen et al., 1994, Paavola et al., 2000a, Paavola et al., 2002b). It includes both objective and subjective measurements and has been shown to have good reliability in healthy individuals and to be able to distinguish patients with ankle ligament injury from healthy individuals. However, to our knowledge, its validity on patients with Achilles tendinopathy has not been evaluated.

The Foot and Ankle Outcome Score (FAOS) is a questionnaire that assesses patients' symptoms, function and foot- and ankle-related quality of life (Roos et al., 2001). This questionnaire has been validated on patients with lateral ankle ligament injuries. Its test-retest reliability was high, but it has not been validated for patients with Achilles tendinopathy (Roos et al., 2001). In a recent study, the FAOS has been used as an outcome measure for treatment in patients with Achilles tendinopathy (Roos et al., 2004).

Stanish and co-workers (2000) devised a system for tendon symptom classification (Table 4). To our knowledge, this classification system has not been evaluated for reliability and validity, but it has been used as an outcome 
measure after surgery in patients who have achillodynia (Rolf and Movin, 1997).

Table 4. Classification system for the effect of pain on athletic performance (Stanish et al 2000).

\begin{tabular}{lll}
\hline Level & Description of pain & Level of sports performance \\
\hline 1 & No pain & Normal \\
2 & Pain only with extreme exertion & Normal \\
3 & Pain with extreme exertion and 1 to 2 hours & Normal or slightly \\
& afterwards & decreased \\
4 & Pain during and after vigorous activities & Somewhat decreased \\
5 & Pain during activity and forcing termination & Markedly decreased \\
6 & Pain during daily activities & Unable to perform \\
\hline
\end{tabular}

The lack of standardized outcome measures for Achilles tendinopathy has caused difficulties when comparing treatment studies (Tallon et al., 2001). In 2001, Robinson and co-workers (2001) developed a questionnaire as an index of the clinical severity of Achilles tendinopathy; the Victorian Institute of Sports Assessment - Achilles questionnaire (VISA-A). This questionnaire is based on a similar questionnaire for patellar tendinopathy, which has been evaluated for reliability and validity and used in research studies of patellar tendinopathy (Visentini et al., 1998, Frohm et al., 2004, Visnes et al., 2005). The VISA-A questionnaire is an easily self-administered questionnaire that evaluates symptoms and their effect on physical activity. It can be used to compare different populations with Achilles tendinopathy and to facilitate comparisons between studies. The VISA-A score has more recently been used to evaluate the outcome of treatment (Peers, 2003a). In the clinic, the VISA-A questionnaire can be used to assess the clinical severity of the patient's symptoms and provide a guideline for treatment, as well as for monitoring the effect of treatment.

\section{Strength and functional tests}

Strength measurements with dynamometry have been performed in patients with Achilles tendinopathy, in both etiological and prospective treatment studies (Niesen-Vertommen et al., 1992, Haglund-Åkerlind and Eriksson, 1993, Alfredson et al., 1998b, Alfredson et al., 1998c, Paavola et al., 2000a, Testa et al., 2002, Mahieu et al., 2005).

Isokinetic dynamometry has been used to test ankle plantarflexion and dorsiflexion strength both concentrically and eccentrically at various angular 
velocities such as $30 \% \mathrm{~s}, 50 \% \mathrm{~s}, 60 \% \mathrm{~s}, 120^{\circ} / \mathrm{s}, 180^{\circ} / \mathrm{s}$, and $225^{\circ} / \mathrm{s}$ (NiesenVertommen et al., 1992, Haglund-Åkerlind and Eriksson, 1993, Alfredson et al., 1998b, Alfredson et al., 1998c, Mahieu et al., 2005). Paavola and coworkers (2000a), on the other hand, measured the isometric strength of the lower limb in an isometric leg press dynamometer. Testa and co-workers (2002) measured maximum isometric muscle activation, as well as isometric muscular endurance, in patients with Achilles tendinopathy.

The various body positions described in the literature and used to measure plantarflexion and dorsiflexion strength are supine with the knee and hip extended, sitting with the hip in 100-110 degrees of flexion and the knee in either 40 degrees or 90 degrees of flexion and a closed-chain position in which the measurement pad was placed on the knee (Niesen-Vertommen et al., 1992, Haglund-Åkerlind and Eriksson, 1993, Alfredson et al., 1998b, Alfredson et al., 1998c, Mahieu et al., 2005, Möller et al., 2005). The reliability of isokinetic and isometric dynamometry is generally high and the various testing positions for plantarflexion and dorsiflexion have shown good test-retest reliability (Alfredson et al., 1998c, Möller et al., 2005). Strength tests are valid for measuring improvements in strength, but they are moderately correlated to functional performance and need to be complemented with other types of functional assessment (Augustsson and Thomeé, 2000).

Muscular endurance testing is another type of muscle function measurement. In a toe-raise test (also called heel-rise or heel-drop), the plantarflexion of the ankle is performed while standing until fatigued. It is the most commonly used test for measuring the muscular endurance of the calf musculature. The normal number of toe-raise repetitions on one leg is regarded to be approximately 25 , but it can range from six to 70 in healthy individuals (Lunsford and Perry, 1995). The testing position for the subject is standing on one leg while maintaining a straight knee, support with the fingertips for balance and avoiding body sway forward. This test has been used in several research studies and has shown good reliability (ICC 0.78 0.84) (Svantesson et al., 1998a, Svantesson et al., 1998b, Möller et al., 2005). The toe-raise test has been used in evaluations of patients with Achilles tendon ruptures, as part of a scoring scale used for patients with Achilles tendinopathy and in one prospective study of Achilles tendinopathy (Paavola et al., 2000a, Möller et al., 2001, Paavola et al., 2002b). Even though toeraises are often prescribed during the rehabilitation of Achilles tendinopathy, they are not commonly used to evaluate the effect of treatment. 
Various jump tests are often used to evaluate function in patients with lower extremity injuries, as well as to evaluate functional performance in athletes (Noyes et al., 1991, Östenberg et al., 1998, Eastlack et al., 1999, Svantesson et al., 2001). Jump tests such as CMJ, SJ and Hopping have been used to evaluate the loading of the Achilles tendon (Komi et al., 1992). Recently, the one-legged hop for distance (standing broad jump) was used in a prospective study to identify intrinsic risk factors for Achilles tendon overuse injury (Mahieu et al., 2005). Even though Achilles tendinopathy is thought to be related to running and jumping, there is a lack of prospective studies of Achilles tendinopathy that evaluate patient function and symptoms when jumping.

\section{Imaging methods}

The imaging modalities that are primarily used in patients with Achilles tendinopathy are ultrasonography (US) and/or magnetic resonance imaging (MRI) (Kader et al., 2002, Paavola et al., 2002a, Bleakney and White, 2005). Regular radiographs are described as being useful in evaluating possible bony abnormalities distally, such as Haglund's deformity (Kader et al., 2002, Schunck and Jerosch, 2005). Imaging is useful for documenting tendon changes, as well as ruling out other causes of patients' symptoms (Shalabi et al., 2001, Kader et al., 2002, Paavola et al., 2002a, Shalabi et al., 2004b, Shalabi et al., 2004c, Bleakney and White, 2005)

US is a quick, safe and inexpensive method, but it is regarded as very user dependent and requires an experienced radiologist (Sandmeier and Renström, 1997). US is valuable for finding the occurrence and location of tendon lesions, but it is unable to differentiate between partial tendon ruptures and focal degenerative areas (Åström et al., 1996, Paavola et al., 1998). Sometimes mild to moderate changes can be found in both symptomatic and asymptomatic tendons and the changes that are seen are not always related to patients' symptoms (Paavola et al., 1998, Fredberg and Bolvig, 2002, Peers et al., 2003b). In a prospective study of the value of US and MRI in assessments of Achilles tendon disorders it was found that US identified abnormal morphology in 37 of 57 symptomatic tendons (65\%) and normal morphology in 19 of 28 asymptomatic tendons (68\%) (Khan et al., 2003b). Furthermore, baseline US findings did not predict the 12-month clinical outcome. Some correlation between the severity of US findings and predictions of recovery has been shown in a retrospective study (Archambault et al., 1998). Abnormalities seen on US in asymptomatic Achilles tendons were related to a $45 \%$ risk of developing symptoms (Fredberg and Bolvig, 2002). More 
recently, US with color Doppler has been used to find areas of neovascularization in the Achilles tendon (Öhberg et al., 2001).

MRI is less user-dependent and is regarded as good at visualizing pathological conditions of the tendon (Sandmeier and Renström, 1997, Kader et al., 2002, Paavola et al., 2002a). Shalabi and co-workers (2001, 2004b) have also used MRI in the follow-ups of patients with Achilles tendinopathy treated with both surgery and exercise.

In their two-year prospective study of the value of US and MRI in assessments of Achilles tendon disorders, Khan and co-workers (2003) found that MRI identified abnormal morphology in 19 of 34 symptomatic tendons (56\%) and normal morphology in 15 of 16 asymptomatic tendons (94\%). Lesser grades of MR signal abnormality at baseline were associated with better clinical status at the 12-month follow-up. Khan and co-workers (2003b) concluded; "Clinicians should exercise discretion in ordering imaging tests and in interpreting their findings in chronic Achilles tendon disorders as both US and MRI commonly result in false positive and false negative diagnosis. As always, careful clinical correlation with imaging findings is essential. Selective use of imaging in patients who fail to respond clinically and who may have clinically underestimated tendon changes may be warranted. Although our findings suggest that imaging adds little information of use for expert sports medicine clinicians in diagnosing tendinopathy, it may useful for inexperienced clinicians who are unsure of their diagnoses and unfamiliar with grading schemes such as VISA-A". 


\section{Treatment}

The literature describes various types of treatment including rest, modalities, orthotics, anti-inflammatory medication, exercise and surgery (Józsa and Kannus, 1997, Kader et al., 2002, Paavola et al., 2002a, Alfredson, 2003c). Despite the high incidence of Achilles tendon disorders and the various recommended treatments only nine randomized treatment trials can be found in the literature (Lowdon et al., 1984, Niesen-Vertommen et al., 1992, Mafi et al., 2001, Silbernagel et al., 2001, Neeter et al., 2003, Peers, 2003a, Paoloni et al., 2004, Roos et al., 2004, Alfredson and Öhberg, 2005d). The type of treatment that is most effective for patients with Achilles tendinopathy and the criteria that should be used when choosing treatment are also unclear (Józsa and Kannus, 1997, Kader et al., 2002, Paavola et al., 2002a, Alfredson, 2003c). A review of various treatment strategies recommended in the literature now follows.

\section{Rest}

Historically, a period of rest from the pain-provoking physical activity, which usually means no running and jumping activity, has been recommended when initiating treatment (Angermann and Hovgaard, 1999, Stanish et al., 2000, Paavola et al., 2000a). Rest is often indicated in acute injuries with active tissue inflammation. As Achilles tendinopathy is an overuse injury, often without inflammatory reactions in the tissue, the need for complete rest can be questioned. The literature often vaguely describes some type of modified rest where the pain-provoking activity should be avoided (Stanish et al., 2000, Kader et al., 2002, Paavola et al., 2002a). Nichols and co-workers (1989) recommend a rest period depending on the severity and duration of injury. Tendon immobilization can, however, be negative for the tendon (Kannus et al., 1997c). Alfredson (2005a, 2005b) reports good effects from the strength training of the tendon, even though it was allowed to be painful. Today the recommendations regarding continued physical activity made by the treating doctor or physical therapist have to be mainly based on the clinicians' personal view and past experience (Wilson and Best, 2005). There is, thus, no prospective study that has evaluated the effect of rest versus continued physical activity. Limiting the patient's physical activity can potentially have a negative impact on his/her health, but, continued abusive physical activity can worsen the injury problems and the recommended appropriate balance is a difficult clinical decision. There is therefore a need for prospective 
randomized treatment studies of the effect of rest and limiting continued physical activity in patients with Achilles tendinopathy.

\section{Exercise}

Muscular strength, power, endurance, flexibility and motor control are important in physical performance and the improvement of these factors is therefore often prescribed in rehabilitation and injury prevention programs for tendon injuries (Hess et al., 1989, Stanish et al., 2000, Cook and Purdam, 2003). Decreased strength has also been implicated as an etiological factor for Achilles tendinopathy. The effects of exercise training appear to be promising and the current consensus appears to be that all patients should be treated with an exercise program for 3-6 months (Niesen-Vertommen et al., 1992, Alfredson et al., 1998b, Angermann and Hovgaard, 1999, Alfredson and Lorentzon, 2000, Mafi et al., 2001, Silbernagel et al., 2001, Kader et al., 2002, Alfredson, 2003c, Roos et al., 2004). Even with other types of treatment such as surgery, sclerosing injections, modalities and medication, some type of exercise is recommended as a complement to the treatment (Schepsis et al., 1994, Paavola et al., 2000b, Testa et al., 2002, Paavola et al., 2002b, Neeter et al., 2003, Alfredson, 2005a). It has been suggested that the eccentric type of strength training programs are particularly effective in improving muscle function and/or aiding in the healing process of the tendon (Fyfe and Stanish, 1992, Alfredson et al., 1998b, Silbernagel et al., 2001, Roos et al., 2004, Öhberg et al., 2004b). The basis of exercise as treatment is to address possible deficiencies in strength, range of motion, balance, proprioception and function. Since the tendon is subjected to the highest loads eccentrically, eccentric training has always been an important part of the prescribed exercise (Fyfe and Stanish, 1992, Giffin and Stanish, 1993, el Hawary et al., 1997, Stanish et al., 2000). Increased speed of movement also increases the load of the tendon during the eccentric muscle activation and, to step up the exercise loads, both the external load and the speed of movement can be increased (Stanish et al., 2000).

The mechanical loading of tendon tissue is important both for maintaining healthy tissue and for tendon healing after injury (Józsa and Kannus, 1997, Kannus et al., 1997c, Ingber, 2005, Kjaer et al., 2005). Exactly which type of exercise, how intense and how often it should be performed to achieve the best outcome for patients with Achilles tendinopathy is not known. In order to improve the treatment (exercise) protocols for this type of injury, further treatment studies that evaluate patient symptoms and function both before and after treatment are needed. 


\section{Non-steroidal anti-inflammatory drugs (NSAIDs)}

Since Achilles tendinopathy is mainly a non-inflammatory condition, apart from a possible short initial inflammatory phase, the use of NSAIDs will have no therapeutic effect (Åström and Rausing, 1995, Alfredson et al., 1999, Khan et al., 1999). NSAIDs might, however, have an analgesic effect on the patient. Åström and Westlin (1992) found no beneficial effect from NSAIDs in patients with Achilles tendinopathy. In a review of the literature, the investigators report insufficient evidence for determining the efficacy of NSAIDs in chronic tendon injury (Almekinders and Temple, 1998).

\section{Corticosteroids}

Corticosteriods are commonly used for their anti-inflammatory action. Corticosteroids are often used by injecting the medication in or around tendons. In a review of the use of corticosteroids in chronic tendon injuries, it was found that they might provide some initial pain relief but that their beneficial effect on the outcome remained uncertain (Almekinders and Temple, 1998). Corticosteroid injections in and/or around tendons have also been associated with tendon ruptures, even though this still remains controversial, and is mostly described in case reports (Kleinman and Gross, 1983, Almekinders and Temple, 1998, Kader et al., 2002, Gill et al., 2004). Cell death and tendon atrophy can be caused by corticosteroids entering the tendon tissue (Fredberg, 1997). However, in a more recent study, intratendinous injections have been performed with reported positive results (Koenig et al., 2004)

An alternative to injections of corticosteroids, is the use of iontophoresis. This is a type of treatment in which electrical stimulation produces a movement between two electrodes and ions diffuse into the tissue (Hasson et al., 1992). The treatment with iontophoresis is sterile, non-invasive and considered to be pain free. Possible side-effects include skin irritation (Harris, 1982).

Contraindications when using iontophoresis are, a pacemaker, pregnancy, diabetes, electrical hypersensitiveness, skin allergies and wounds (Harris, 1982). Further prospective randomized studies of patients with Achilles tendinopathy are needed before it can be determined whether corticosteroids are useful and/or harmful for patients with acute Achilles tendinopathy. 


\section{Sclerosing injections}

Ultrasonography guided sclerosing of neovessels in patients with Achilles tendinopathy is a fairly new type of treatment (Öhberg and Alfredson, 2002). The treatment is based on the finding of neovascularization in painful tendons when using high-resolution color Doppler ultrasound. The theory is that the neovascularization is the cause of the pain (Öhberg and Alfredson, 2002). In a double-blind, randomized, controlled trial, 5/10 patients receiving sclerosing injections were satisfied with the treatment, while no one in the control group was satisfied (Alfredson and Öhberg, 2005d). The same research group report that, at the same follow-up time (3 months), all their patients receiving heavyload eccentric calf muscle training were satisfied with the treatment (Alfredson et al., 1998b). Eccentric training is described as causing the blockage of the neovessels in the same way the sclerosing injections are (Öhberg and Alfredson, 2004a). The patients receiving sclerosing injections are also allowed gradually to increase tendon-loading activity after 1-3 days of rest (Alfredson, 2005b). It is therefore difficult to exclude the possibility that some of the positive effects are due to the tendon loading activity.

\section{Orthotics}

It has been suggested that anatomical malalignments are risk factors for Achilles tendinopathy. Corrective orthotics are therefore often prescribed for patients with Achilles tendinopathy. Clinically, some patients report good effects, whereas some claim no effects from custom-made orthotics. Until there is research showing that anatomical malalignment causes Achilles tendinopathy and that corrective orthotics are effective in relieving symptoms, the use of corrective orthotics should be based on clinical judgement. There is one double-blind study, which showed no benefit from using viscoelastic heel pads on Achilles tendinopathy (Lowdon et al., 1984).

\section{Other treatments}

Modalities such as therapeutic ultrasound, laser, electrical stimulation, and acupuncture are often used by physical therapists. These modalities are, however, mostly used in order to reduce the pain making it possible for the patients to perform therapeutic exercises in order to improve the strength and function of muscles and tendon tissue (Rivenburgh, 1992, Stanish et al., 2000). Therapeutic ultrasound in animals has shown some beneficial effect on tendon tensile strength, but there are no data on the effects in humans (Enwemeka, 1989a). Deep friction massage is sometimes described as breaking down scar tissue in tendon injuries, but it is often very painful and 
its positive effect is questionable (Stanish et al., 2000, Cook and Purdam, 2003). Shock-wave therapy (which uses high-energy sound waves) has also been suggested to have positive effects on patients with tendinopathy. Concerning treatment with shock-wave therapy for patients with Achilles tendinopathy, there are conflicting reports of both positive effects and no effects related to the treatment (Peers, 2003a, Costa et al., 2005)

\section{Surgery}

There appears to be consensus in the literature that surgery should only be performed after patients have been treated unsuccessfully with a treatment protocol involving exercise for a minimum of 6 months (Khan et al., 1999, Kader et al., 2002, Paavola et al., 2002a, Wilson and Best, 2005). The surgical techniques vary but usually include removing abnormal tendon tissue and releasing adhesion and scarring (Kader et al., 2002, Paavola et al., 2002a, Wilson and Best, 2005). It is not clear why surgery sometimes helps and the post-operative rehabilitation may be as important as the actual surgery (Khan et al., 1999). The success rates for surgery are reported to be approximately 70-80\% (Nelen et al., 1989, Leach et al., 1992, Schepsis et al., 1994, Tallon et al., 2001, Paavola et al., 2002a, Paavola et al., 2002b, Alfredson and Öhberg, 2005c). In a review of the outcome of surgery for chronic Achilles tendinopathy, a negative correlation was found between reported success rates and overall method scores (Tallon et al., 2001). Complications after surgery also need to be considered and, in a study of 432 consecutive patients, there were $46(11 \%)$ complications from treatment (Paavola et al., 2000b). With improvements in non-surgical treatment, there will, however, be fewer requests for surgery.

\section{Prevention}

The prevention of injuries remains an important goal for both clinicians and researchers. In the literature, it is suggested that tendinopathy can be prevented by encouraging athletes and coaches to follow sensible training programs (Robinson et al., 2001, Kader et al., 2002). The appropriate training level for each individual is not known and recommendations are usually based on empirical evidence. Prevention nowadays is mostly geared towards avoiding and limiting suggested predisposing factors. In order to develop appropriate prevention protocols, further studies of injury risk factors and the way the injury affects the function in patients are needed (Hess et al., 1989, Murphy et al., 2003). Proper research on Achilles tendinopathy prevention 
can then be conducted. To our knowledge, there are currently no welldesigned studies of the prevention of Achilles tendinopathy.

\section{Prognosis}

The natural course of Achilles tendinopathy is not fully known. The shortterm (up to 12 months) prognosis following non-surgical treatment is reported to be good, with a success rate of $50-100 \%$ (Alfredson et al., 1998b, Angermann and Hovgaard, 1999, Öhberg and Alfredson, 2003, Alfredson and Öhberg, 2005d). Angermann and Hovgaard (1999) report that, at follow-up 33-72 months after non-surgical treatment, $65 \%$ of the patients had improved or been cured. In a recent 8 -year follow-up study of patients, initially treated non-surgically, 94\% were asymptomatic (Paavola et al., 2000a). Twenty-nine percent had failed to respond to non-surgical treatment and had undergone surgery. Others report that $24-45 \%$ of patients fail to respond to non-surgical treatment and will undergo surgery (Kader et al., 2002). It is also reported, in the 8 -year follow-up, that $41 \%$ of the patients developed symptoms on the previously uninjured side (Paavola et al., 2000a). The success rates for surgery are reported to be approximately $70-80 \%$ (Nelen et al., 1989, Leach et al., 1992, Schepsis et al., 1994, Tallon et al., 2001, Paavola et al., 2002a, Paavola et al., 2002b, Alfredson and Öhberg, 2005c). Today fewer patients may need surgery due to improved non-surgical treatment, such as exercise treatment. 


\section{Summary of problem areas presented in the introduction}

\section{General problem area}

- Vigorous physical activity, or periods of inactivity followed by intense physical activity, are major causes for various overuse injuries to the human body. One of the most common overuse injury is Achilles tendinopathy, which causes pain and impaired physical performance. It is described as occurring primarily in middle-aged men, but it occurs in both men and women of all ages. The injury causes patients to limit their physical activity level, often for long periods, with a potential negative impact on their health.

\section{Specific problem areas}

- There is a lack of standardized outcome measures for patient symptoms and impairments in physical activity.

There is a need for outcome measures, which can be used in both research and clinical settings. A good outcome measure should be able to measure the patient's clinical severity, as well as being used to evaluate the effect of different treatment. An outcome measure of this kind would facilitate comparisons of the effect of various treatment protocols.

- There is a lack of validated and reliable methods for evaluating lower leg function in patients with Achilles tendinopathy.

Achilles tendinopathy appears to cause difficulties with performing physical activity in the active population. Exactly how it affects the lower leg muscle-tendon functions is, however, not known.

- There is a lack of treatment protocols, for patients with Achilles tendinopathy, which are evaluated and proven effective in randomized controlled studies.

The literature describes various types of treatment including rest, modalities, anti-inflammatory medications, exercise and surgery. It is, however, unclear which type of treatment is most effective for patients with Achilles tendinopathy and which criteria should be used when choosing treatment. 


\section{Aims of the thesis}

The overall aim of this thesis was to develop and evaluate outcome assessments and treatment protocols for patients with Achilles tendinopathy. The specific purposes of each study were as follows.

\section{Study I}

The purpose of Study I was to examine the reliability of measurement techniques and evaluate the effect of a treatment protocol including eccentric overload for patients with chronic pain from the Achilles tendon.

\section{Study II}

The purpose of Study II was to compare the effects of iontophoresis with dexamethazone to iontophoresis with saline solutions in patients who had acute (less than 3 months) pain from the Achilles tendon, in terms of range of motion, muscular endurance, pain and symptoms.

\section{Study III}

The purpose of Study III was to cross-culturally adapt the VISA-A questionnaire to Swedish and to perform reliability, validity and structure evaluations.

\section{Study IV}

The purpose of Study IV was to evaluate whether Achilles tendinopathy caused functional deficits on the injured side compared with the non-injured side in patients. A test battery comprised of tests for different aspects of muscle-tendon function of the gastrocnemius, soleus and Achilles tendon complex was developed to evaluate lower leg function. The test battery's testretest reliability and sensitivity (the percentage probability that the tests would demonstrate abnormal lower limb symmetry index in patients) were also evaluated.

\section{Study V}

The purpose of Study V was to demonstrate whether continued Achilles tendon loading activity, with the use of a pain monitoring model, during rehabilitation will result in significant improvements in patients' symptoms and function, and whether this training regimen could have any negative effects on the final outcome in comparison with patients resting from such activity. 


\section{Subjects}

\section{Table 5. Summary of subjects included in the studies}

\begin{tabular}{|l|c|c|c|c|c|c|}
\hline & Study I & Study II & \multicolumn{2}{|c|}{ Study III } & \multicolumn{2}{c|}{ Studies IV and V } \\
\cline { 4 - 7 } & Patients & Patients & Healthy & Patients & Healthy & Patients \\
\hline & 40 & 25 & 15 & 51 & 15 & 42 \\
$\mathrm{n}$ & 31 & 15 & 12 & 32 & 12 & 23 \\
Men & 9 & 10 & 3 & 19 & 3 & 19 \\
Women & & & & & & \\
\hline Age (years) & 44.5 & 38 & 29.5 & 43.1 & 30 & 46 \\
Mean & 13.0 & 13.1 & 4.3 & 14.5 & 2 & 8 \\
SD & $19-77$ & $18-76$ & $23-37$ & $18-65$ & $23-38$ & $30-58$ \\
Range (min-max) & & & & & & \\
\hline Duration of symptoms & & & & & & \\
(months) & 29 & $<3$ & N/A & 31.8 & N/A & 37 \\
Mean & 41.5 & & & 90.8 & & 64 \\
SD & 11 & & & 6 & & 9.5 \\
Median & $4-192$ & & & $1-480$ & & $3-360$ \\
Range (min-max) & 57 & 25 & & & & \\
\hline Involved tendons (n) & 10 & 13 & N/A & & N/A & 12 \\
Right & 13 & 12 & & & & 16 \\
Left & 17 & 0 & & 15 & & 14 \\
Both & & & & & \\
\hline
\end{tabular}

\section{Study I}

The patients were recruited through mailings to hospitals and clinics, as well as sport clubs in Göteborg. Men and women above the age of 18 years, with chronic pain from the Achilles tendon (proximal achillodynia), with a duration of pain of more than three months, were included in the study. The exclusion criteria were surgery on the involved foot, history of rheumatoid arthritis, diabetes, or any other illness that was thought to interfere with the study and/or if they were currently receiving treatment for Achilles tendon disorders.

Forty-nine patients (13 women and 36 men) with chronic proximal achillodynia were included in the study. Twenty of these patients had bilateral problems. To establish reliability measures, 32 (44 involved Achilles tendons) of the 49 patients who were initially included were evaluated twice before treatment. The subjects in the reliability-testing group consisted of 8 women and 24 men; their mean $( \pm \mathrm{SD})$ age was $42( \pm 13.9)$ years. Nine patients $(12$ 
Achilles tendons), four women and five men, withdrew from the treatment study. Three of them belonged to the experimental group and they stopped at their own request within two weeks of the start of the study. The other six belonged to the control group. Two of them decided to have surgery after six weeks, one patient underwent surgery for bursitis, one was not able to attend the evaluation and two withdrew without specifying the reason.

The remaining 40 patients, with a total of 57 involved Achilles tendons, are included in the results. The experimental group consisted of 22 patients ( 5 women and $17 \mathrm{men}$ ), with a total of 30 involved Achilles tendons. The control group consisted of 18 patients (4 women and 14 men), with a total of 27 involved Achilles tendons. The age of all the patients in the study ranged from 19 to 77 years, with a mean age of 45 years. The median (mean) duration of symptoms in the experimental group was 9 months (21 months), with 17 (77\%) patients having a duration of symptoms of less than two years. In the control group, the median (mean) duration of symptoms was 18 months (41 months), with $11(61 \%)$ patients having a duration of symptoms of less than two years. In the experimental and the control group, $11(50 \%, 58 \%)$ patients currently (or used to) ran/jogged on a regular basis. All the other patients, apart from two, reported being involved in some type of exercise activity (ice hockey, soccer, aerobics and walking). No significant differences were noted between the groups in terms of gender, age, height, duration of symptoms or involvement in sports.

\section{Study II}

The patients were recruited through mailings to hospitals and clinics, as well as sports clubs in Göteborg, at the same time as the subjects in Study I. The study population consisted of men and women above the age of 18 years. The inclusion criteria were acute pain from the Achilles tendon with onset less than three months before with the pain located two to six $\mathrm{cm}$ proximal to the calcaneal insertion. The exclusion criteria were surgery on or previous injury to the involved foot, a history of rheumatoid arthritis, diabetes, or any other illness that was thought to interfere with the study.

A total of 25 patients were included and randomly allocated to an experimental group or a control group. The experimental group consisted of 14 patients ( 5 women and 9 men) with a mean $( \pm \mathrm{SD})$ age of $38( \pm 15.6)$ years and a range of 18-76. The control group consisted of 11 patients (5 women and 6 men) with a mean $( \pm \mathrm{SD})$ age of $39( \pm 3.9)$ and a range of 21-52. 


\section{Study III}

For test-retest evaluation, we recruited a convenience sample of 15 healthy individuals ( 3 women and 12 men) with a mean $( \pm$ SD) age of $29.5( \pm 4.3)$ years.

Fifty-one patients (19 women and 32 men) with a mean $( \pm \mathrm{SD})$ age of 45.4 $( \pm 15.5)$ years, with Achilles tendinopathy, were included in the reliability evaluation for internal consistency and the validity evaluations. The mean $( \pm \mathrm{SD})$ duration of symptoms for the 51 patients was $55.5( \pm 134.7)$ months. Twenty-two ( 8 women and 14 men) of the 51 patients also participated in a test-retest evaluation. Bilateral symptoms were reported in 15 of 51 patients (7 of the 22 in test-retest group).

The patients were recruited from 11 physical therapy clinics throughout Sweden. The inclusion criteria were the same as in the original study (Robinson et al., 2001). The subjects had to be older than 18 and be able to give written consent. The subjects had to have a diagnosis of Achilles tendinosis, paratendinitis, or partial rupture with or without retrocalcaneal or Achilles bursitis. The diagnosis was based on patient history and the physical therapists' clinical findings. Subjects with total Achilles tendon rupture and pregnant or nursing women were excluded.

\section{Study IV}

To establish reliability measures, a convenience sample of 15 healthy individuals (12 men and 3 women) was recruited. The inclusion criteria for these subjects were healthy individuals, aged 20-40. The exclusion criteria were individuals with any foot, knee, hip and back injury which could prevent participation in the study. Elite athletes (competing in the two highest divisions in the specific sport) were also excluded. The mean age was 30 years (SD 12, range 23-38), the mean height was $181 \mathrm{~cm}$ (SD 10.9, range 163-206) and the mean weight was $78 \mathrm{~kg}$ (SD 12, range 59-95).

The patients were recruited through mailings to hospitals, orthopedic surgeons, physical therapy clinics and orthopedic technicians in the greater Göteborg area. They were referred by their doctor or initiated contact themselves. Men and women, 20-60 years of age, with Achilles tendinopathy and duration of pain of more than 2 months were included. The definition of Achilles tendinopathy was the clinical diagnosis of a combination of Achilles tendon pain, swelling and impaired performance, as described in the literature (Kader et al., 2002, Paavola et al., 2002a, Maffulli et al., 2003a). The exclusion criteria were injury to foot, knee, hip or back and/or history of rheumatoid arthritis or any other illness or injury thought to interfere with 
participation in the study. Patients with insertional tendinopathy were also excluded.

A total of 42 patients (19 women and 23 men) with Achilles tendinopathy were included. Fourteen of these patients had bilateral problems resulting in a total number of 56 involved tendons. Their mean age was 46 (SD 8, range 3058) years and their mean duration of symptoms was 37 (SD 64, range 3-360) months. Their mean $( \pm \mathrm{SD})$ height and weight were $178( \pm 8.5)$ and 80.7 $( \pm 13.1)$ respectively. Eighty-three percent of the patients reported being injured in connection with exercise and $88 \%$ of the patients reported that the injury was due to overuse.

\section{Study V}

The same patients as in Study IV were included in Study V. They were randomly allocated into two treatment groups. Two of the patients included in the Exercise training group were excluded after the initial evaluation. One was excluded for not being able to attend any of the other evaluations or physical therapy visits because of illness in the family and the other patient developed pain in his ankle and knee which hindered his participation in the study. Two patients in the Active rest group were also excluded after the initial evaluation. One was excluded at his/her own request because of selfreported non-compliance and difficulty attending evaluations and physical therapy visits due to work. The other patient was excluded because of illness which prevented him from starting treatment or attending the 6-week and 3month evaluations.

A total of 38 patients (18 women, 20 men) with a total of 51 injured tendons were included in the study. The Exercise training group consisted of 19 patients ( 7 women, 12 men) with a total of 26 injured tendons. Their mean $( \pm \mathrm{SD})$ age and duration of symptoms were $44( \pm 8.8)$ years and $48( \pm 84.5)$ months respectively. The Active rest group consisted of 19 patients (11 women, 8 men) with a total of 25 injured tendons. Their mean $( \pm \mathrm{SD})$ age and duration of symptoms were $48( \pm 6.8)$ years and $24.4( \pm 40.8)$ months respectively. There were no significant differences between the groups in terms of age, height, weight, duration of symptoms and physical activity level (Grimby, 1986). The majority of the patients reported that the injury was due to overuse (87\%) and being injured doing exercise (84\%). 


\section{Ethics}

All the subjects received oral and written information about the purpose and procedure of the study and written informed consent was obtained. Ethical approval was obtained from the Human Ethics Committee at the Medical Faculty, Göteborg University, Sweden. 


\section{Methods}

\section{Evaluations}

\section{Studies I and II}

\section{Questionnaire}

The questionnaire included questions about physical activity level, work, other injuries, previous treatment for the Achilles tendon disorder and medication. It also assessed the start and duration of the Achilles tendon disorder, how it affected the patient's activity level and how it affected walking, walking up or down stairs and running. Questions relating to symptoms, such as morning stiffness and swelling, were also included. At the one-year follow-up, a separate questionnaire was used with questions relating to the patients' perception of the success of the treatment protocol and whether they felt they had fully recovered.

\section{Ankle range of motion test}

Active range of motion of ankle dorsiflexion and plantarflexion were measured with the patient placed supine on a treatment table. The fibular head, the most prominent point of the lateral malleoli and the head of the fifth metatarsal were palpated and marked with an ink marker. After the standardized stabilization of the lower leg, the patient was asked to "move the foot upwards as far as possible" (dorsiflexion) and to hold it for a few seconds and then to "move the foot downwards" (plantarflexion) as far as possible and to hold it for a few seconds. This procedure was videotaped with a VHS video camera (Panasonic). The angle between the reference points was measured on a TV screen using a standard goniometer. The range of motion was given based on 0 degrees being the 90 -degree angle between the estimated line from the head of fibula to the lateral malleoli and the line between the lateral malleoli and the head of the fifth metatarsal.

\section{Pain and palpation}

The patient was asked to indicate the painful area with a circle on an anatomical drawing of the lower leg from the posterior view. The patient marked the most painful location with an X. This was done both before and after each evaluation. There was a Visual Analog Scale (VAS) underneath each of the pain drawings and the patient was also asked to indicate with an $\mathrm{X}$ the pain at rest at that moment, using the VAS. A VAS from 0 to $100 \mathrm{~mm}$ was used, with 0 being no pain and 100 being the worst imaginable pain 
(Carlsson, 1983, Price et al., 1983). The Achilles tendon was palpated through a pinch grip between the thumb and index finger to find the most tender/painful point (Figure 12). This location was marked with an ink marker. The painful location was pinched for a few seconds with a force equal to the force needed to hold a $1 \mathrm{~kg}$ weight between the thumb and index finger. The patient was then asked to rate the pain caused by the pinching on a VAS. After the pinching, the patient was asked to stand up with his/her back and heels against a wall and the feet together. The location of the painful area was measured in centimeters as the distance between the floor and the ink mark on the Achilles tendon.

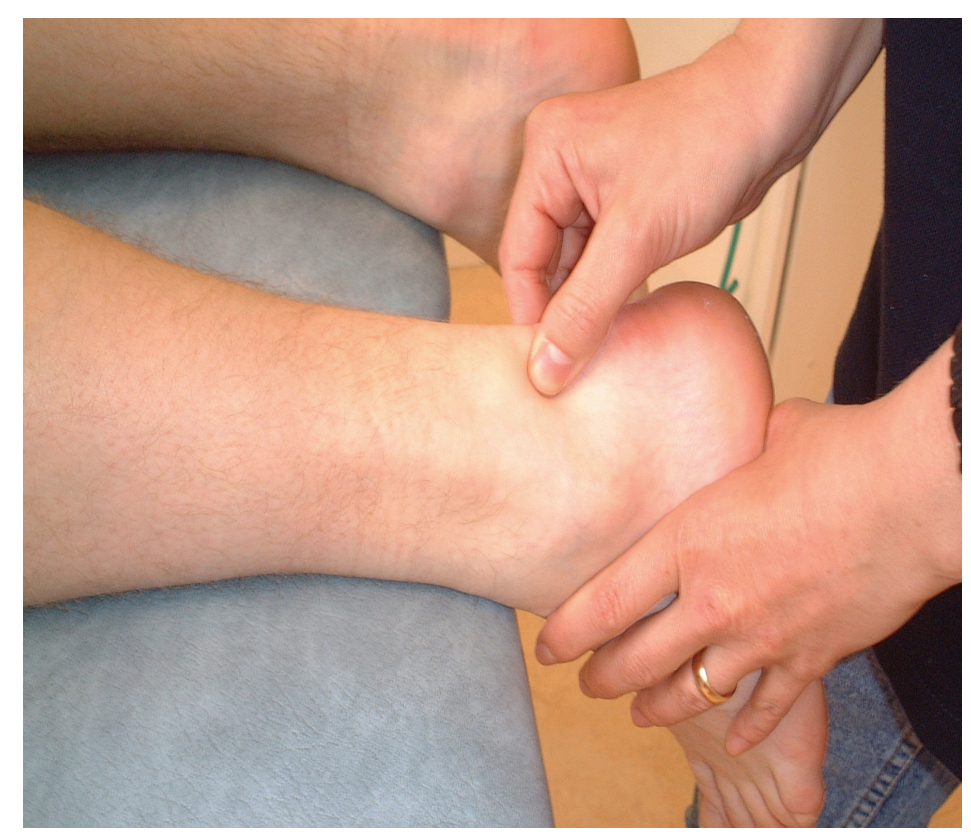

Figure 12. The palpation test; a pinch grip is used.

\section{Toe-raise test}

The equipment used for the toe-raise test was two high jump stands, a wooden bar placed between the two stands and a metronome (Figure 13). First, the height of the patient was measured $(\mathrm{cm})$. The maximum height of a onelegged toe-raise was then measured by having the patient stand barefoot with his/her big toe on a line on the floor, which was directly under the bar, and then perform the highest possible toe-raise. The height between the head and the bar was measured with a ruler before the toe-raise and when the patient was at the highest point. The height of the maximum toe-raise was then calculated in centimeters and $90 \%$ of this height added to the height of the patient was used for the toe-raise test. The same test height was then used for each subsequent testing occasion. 
The patient was asked to perform as many toe-raises as possible at a frequency of 25 toe-raises per minute. A metronome set at 50 beats a minute kept the frequency. The patient went up on one beat and down on the next. The patient was allowed to have two fingers at shoulder height against the wall for balance. The test was discontinued when the patient did not reach the bar, could not maintain the frequency, could not keep the knee straight, or asked to stop due to pain or fatigue. The number of toe-raises was recorded, as well as the reason for ending the test. Immediately after the test, the patient was asked to report the amount of pain, on the VAS, from the Achilles tendon.

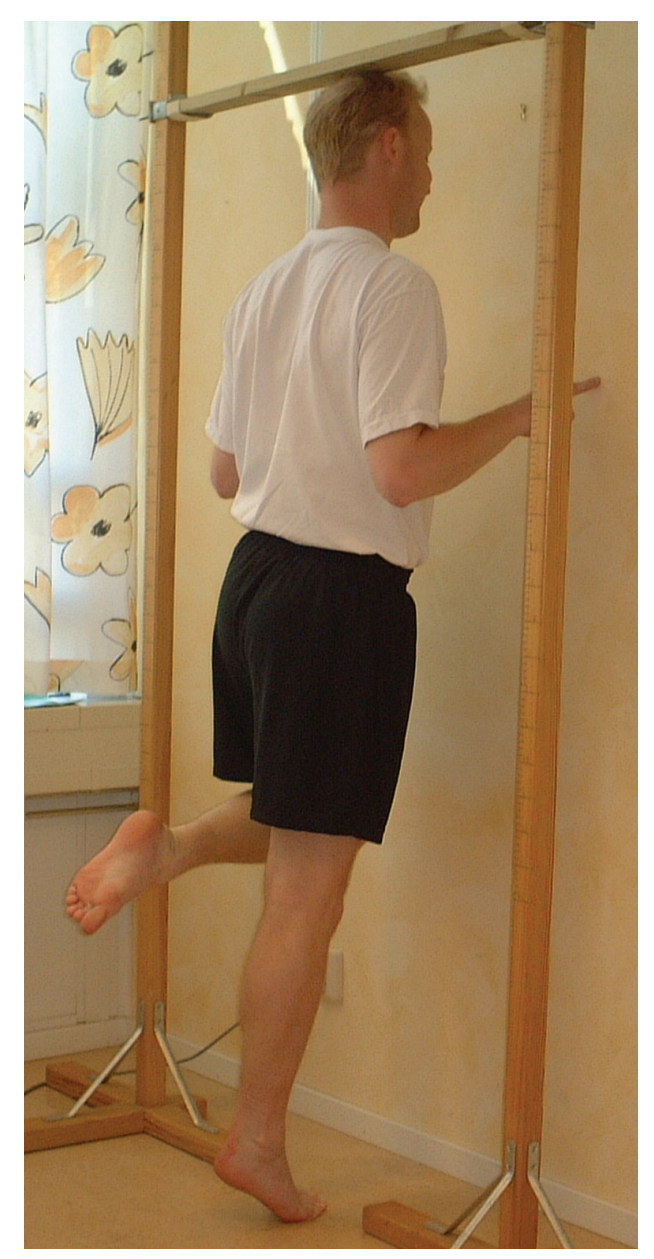

Figure 13. The toe-raise test.

\section{Study I}

\section{Jumping test}

The patient performed a one-legged counter-movement jump on a jump mat (Tapeswitch Corporation of America). The amount of time the patient was in the air was measured to a thousandth of a second and the main unit (Eleiko 
Sport) then calculated the height in centimeters based on the time in the air. Earlier studies have shown that the jump mat has a methodological error of 23\% (Komi and Bosco, 1978, Anderson and Pandy, 1993). After information, demonstration and familiarization with the counter-movement jump, the patient performed one or two submaximum jumps prior to the actual testing. The counter-movement jump was started in the standing position. The patient then quickly squatted down (i.e. bent the knee, hip and ankle) before jumping up. Three jumps were performed and the highest jump was recorded. Immediately after the test, the patient reported the pain, on the VAS, from the Achilles tendon during the jump.

\section{Study III}

\section{Cross-cultural adaptation}

To establish good face validity and content validity, the translation and cultural adaptation of the VISA-A questionnaire into Swedish was performed in several steps (Beaton et al., 2000). The English version (appendices B) was translated into Swedish independently by three people. All three were working in the medical field and had English as a second language. The three translations were synthesized into one Swedish version by a panel of experts consisting of four physical therapists who specialize in musculoskeletal disorders. The back translations of the Swedish version into English were performed by another three people. Two of the back translators were in the medical field (a sports medicine doctor and a physical therapist), while the third person was Swedish but had lived in the USA for many years. The panel of experts (same as above) then compared the original version with the back translations. The panel of experts consolidated the various versions into one pre-final version of the VISA-A questionnaire - Swedish version (VISA-A-S). The pre-VISA-A-S was pilot tested on five patients and five healthy individuals. After pilot testing Question 1 was made clearer by entering the minutes in the boxes.

The final version of the VISA-A-S (appendices A) was tested on both healthy individuals and patients with a diagnosis of Achilles tendinopathy. For the test-retest evaluation, we recruited a convenience sample of 15 healthy individuals aged 20-40 years. They completed the VISA-A-S questionnaire three times within two weeks. The questions were answered with respect to their right Achilles tendon. Fifty-one patients with Achilles tendinopathy were included in the reliability evaluation for internal consistency and the validity evaluations. Twenty-two of the 51 patients also participated in a test-retest evaluation. At their first physical therapy visit, all 
the patients completed questionnaires relating to their injury, physical activity (Grimby, 1986), tendon injury classification according to Stanish and coworkers (2000) and one VISA-A-S questionnaire for each leg. For the patients with bilateral symptoms, the side with the lowest VISA-A-S score or if the scores were equal, the side with the longest duration of symptoms was chosen for the evaluations. The 22 patients that participated in the test-retest evaluation completed the VISA-A-S questionnaire a second time within a week of the first visit.

The construct validity of the VISA-A-S was tested according to the original article on the VISA-A English version (Robinson et al., 2001). The results from the 51 patients who completed the VISA-A-S questionnaire were compared with the results from the tendon grading system by Stanish and coworkers (1984). The results from the VISA-A-S questionnaire for patients with Achilles tendinopathy were also compared with the results for healthy individuals.

The criterion validity of the VISA-A-S questionnaire was evaluated by comparing the results for our patients $(n=51)$ with the results for the two patient groups, the non-surgical group $(n=45)$ and surgical group $(n=14)$, in the original article by Robinson and co-workers (2001). The results for the healthy individuals in our study were also compared with the results for the healthy individuals in the original study.

The structure of the VISA-A-S questionnaire was evaluated with a factor analysis. 


\section{Studies IV and V}

\section{Questionnaire}

The 42 patients were evaluated using the Swedish version of the Victorian Institute of Sports Assessment-Achilles questionnaire (Robinson et al., 2001, Silbernagel et al., 2005) and a physical activity questionnaire (Table 6) (Grimby, 1986).

Table 6. Physical activity questionnaire (Grimby, 1986).

\begin{tabular}{|c|l|}
\hline 1 & Hardly no physical activity. \\
\hline 2 & Mostly sitting, sometimes a walk, easy gardening or similar tasks. \\
\hline 3 & $\begin{array}{l}\text { Light physical exercise around 2-4 hours a week, e.g. walks, } \\
\text { fishing, dancing, ordinary gardening, including walks to and from } \\
\text { shops. }\end{array}$ \\
\hline 4 & $\begin{array}{l}\text { Moderate exercise 1-2 hours a week, e.g. jogging, swimming, } \\
\text { gymnastics, heavier gardening, home-repairing or easier physical } \\
\text { activities more than 4 hours a week. }\end{array}$ \\
\hline 5 & $\begin{array}{l}\text { Moderate exercise at least 3 hours a week, e.g. tennis, swimming, } \\
\text { jogging etc. }\end{array}$ \\
\hline 6 & $\begin{array}{l}\text { Hard or very hard exercise regularly and several times a week, } \\
\text { where the physical exertion is great, e.g. jogging, skiing. }\end{array}$ \\
\hline
\end{tabular}

\section{Pain evaluation}

Pain on palpation of the Achilles tendon and the patients' report of pain during the test were also documented. For pain documentation, the Visual Analog Scale (VAS), where 0 is equal to no pain and 10 is the worst pain imaginable, was used.

\section{Ultrasonography measures}

In order to establish a diagnosis of Achilles tendon pathology, Ultrasonography was performed using a real-time scanner, with a $7.5 \mathrm{MHz}$ linear array probe. Both tendons were scanned in all patients, in the longitudinal as well as the axial plane. The tendon pathology was registered as heterogeneity and/or hyperechoic areas in the tendon. Tendon oedema and tendon thickening were also documented. 


\section{Functional evaluation}

The evaluation consisted of calf circumference measurements, range of motion measurements (dorsiflexion when standing), three different jump tests, and three different strength tests. All the evaluations were performed by the same physical therapist. The patients' height and weight were measured and documented. The tests in the test battery were always performed in the same order and the right side was always tested first. The reason for this was to eliminate variations in results due to variations in fatigue caused by previous tests and to simplify the testing methods so that the procedure would always be the same on each test occasion. All the subjects were given standardized instructions and the tests were then demonstrated by the tester. The subjects also performed 3-5 practice trials prior to testing. Verbal encouragement was used and athletic footwear was standardized. Prior to testing, the patients warmed up by cycling for five minutes on a stationary bicycle (Monark ${ }^{\circledR}$ ), followed by 3 sets of 10 two-legged toe raises.

The MuscleLab ${ }^{\circledR}$ (Ergotest Technology, Oslo, Norway) measurement system was used for the evaluations (Figure 14). MuscleLab ${ }^{\circledR}$ is a data collection unit to which sensors of different kinds can be connected. For the jump tests, a "contact/light" mat, consisting of a field of infrared light beams, approximately $4 \mathrm{~mm}$ above the floor, connected to a timer that was triggered when the light beams were interrupted was used (Figure 15). The response time is better than or equal to $2 \mathrm{~ms}$. Using this set-up, ground contact time and air flight times were recorded and the jump height in centimeters was calculated by the system. For the strength tests a linear encoder was used (Figure 16). A spring-loaded string is connected to a sensor inside the linear encoder unit. When the string is pulled, the sensor emits a series of digital pulses that are proportional to the distance traveled. The resolution is approximately one pulse every $0.07 \mathrm{~mm}$. By counting the number of pulses/time, the displacement as a function of time can be recorded and thus enable the calculation of velocity, force and power (force times velocity). In this experiment the spring-loaded string of the linear encoder was attached to the heel of the subject's shoe. 


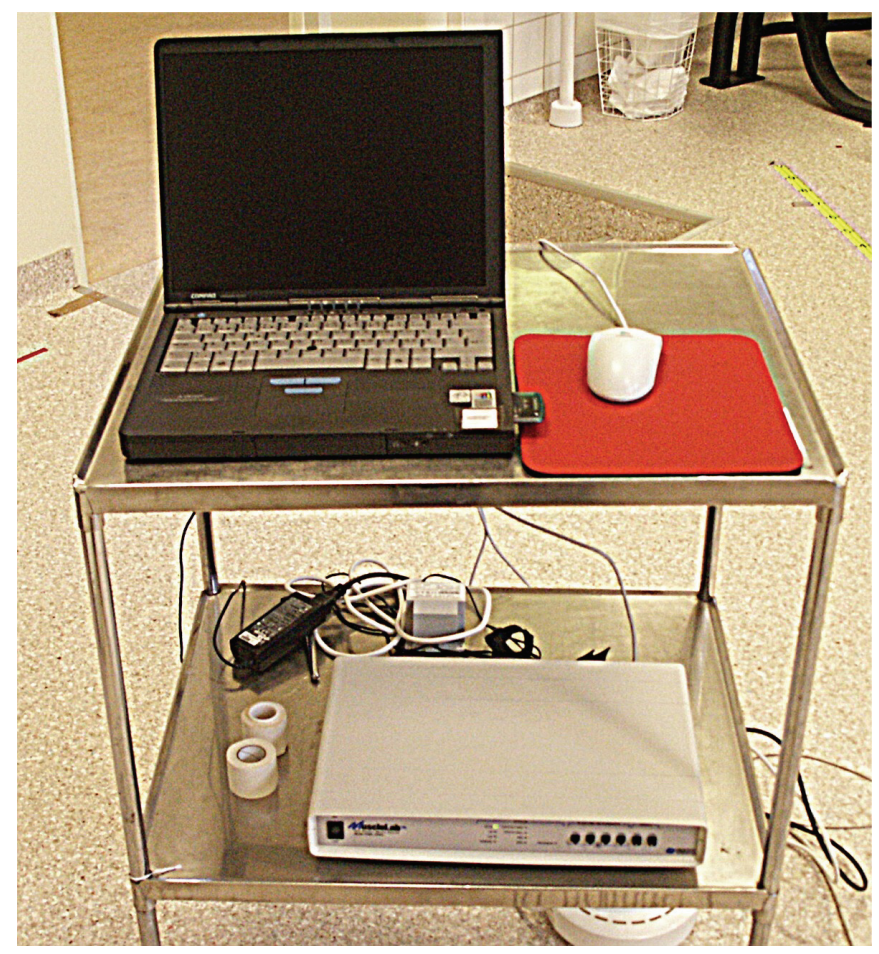

Figure 14. The MuscleLab ${ }^{\circledR}$ unit

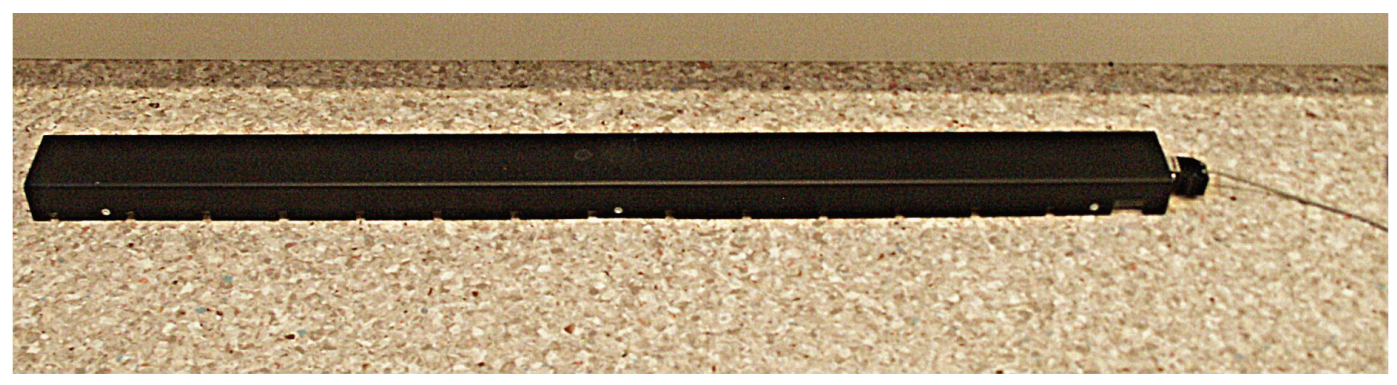

Figure 15. The "contact/light" mat

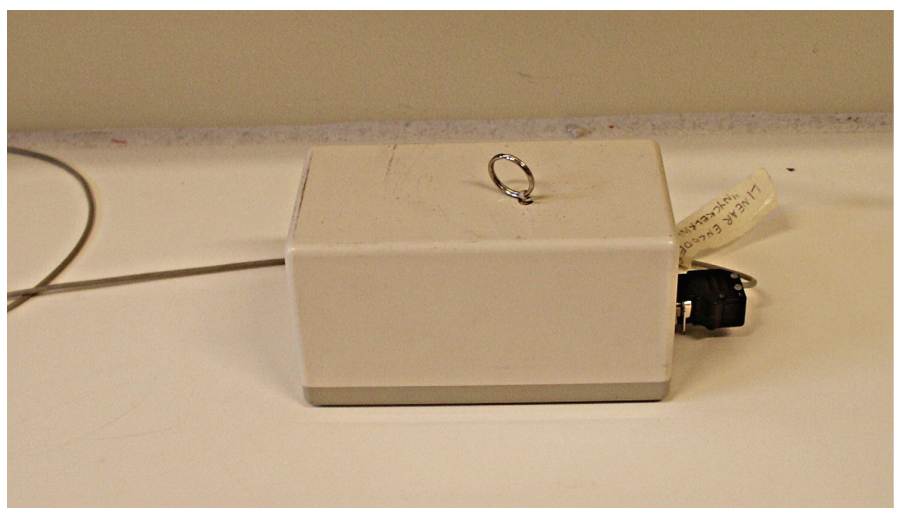

Figure 16. The linear encoder unit 


\section{The jump tests}

1. Vertical jump - counter movement jump (Figure 17). The vertical jump test was performed as a one-legged counter movement jump (CMJ). The starting position was an upright position with the hands placed behind the back. The subjects quickly bent their knee as much as they wanted and then immediately jumped upwards attempting to maximize the height they jumped. The subjects performed three maximum approved trials for each leg, starting with the right and alternating between the right and the left leg. However, if the subject increased his/her hop performances in all three jumps, additional jumps were performed until no increase

\section{Drop jump followed by a} vertical jump on one leg (drop CMJ) (Figure 18). The subjects started by standing on one leg on a wooden box $(30 \mathrm{~cm}$ high for healthy subjects and $20 \mathrm{~cm}$ high for patients). The subjects were instructed to "fall" down onto the floor and, directly on landing perform a maximum vertical onelegged jump. The subjects had their hands on their back throughout the test. After trial jumps, the subjects started on the right leg and alternated legs until three approved jumps per leg were performed. The contact time and jump height of the was seen. The best jump height for each leg was used for data analysis.

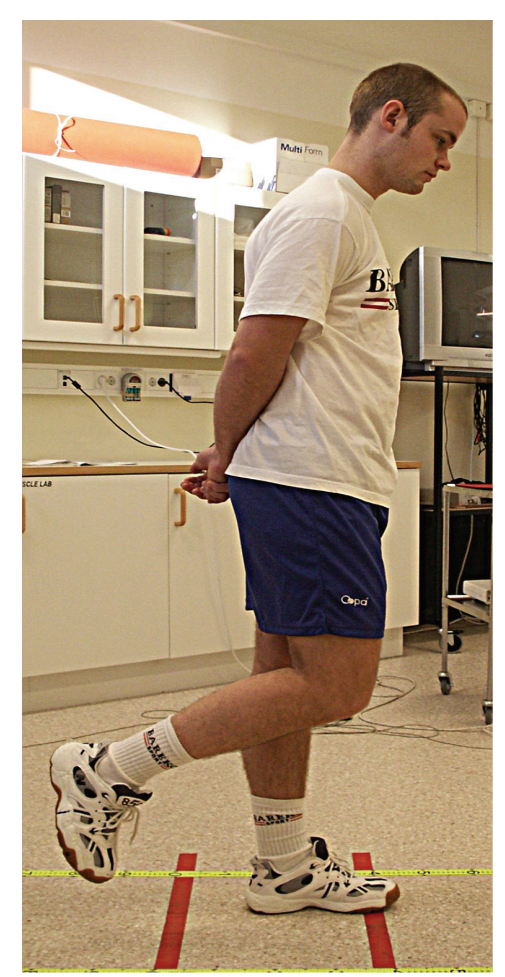

Figure 17. Counter movement jump

highest jump for each leg were used for data analysis.

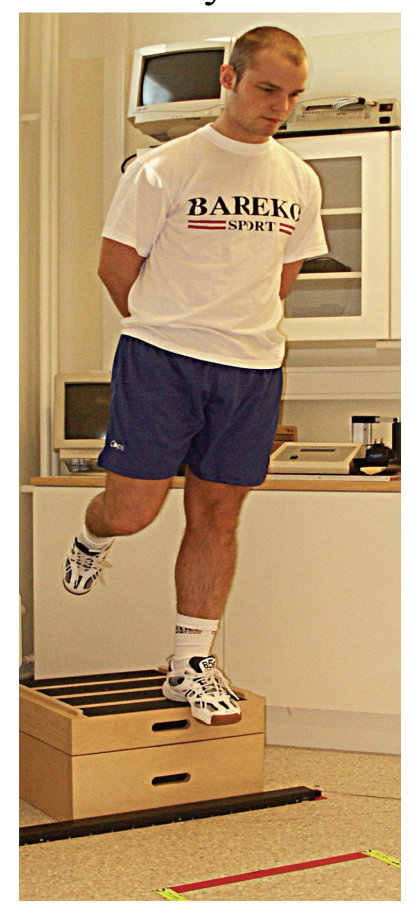

Figure 18. Drop CMJ 
3. Hopping (Figure 19). Hopping is a continuous rhythmical jump, similar to jumping rope (Jones and Watt, 1971, Komi et al., 1992, Fukashiro et al., 1995b, Funase et al., 2001). The purpose of this test, involving the SSC of the Achilles tendon and calf musculature, is to measure the ability of the muscletendon complex to utilize its elastic properties (Komi et al., 1992, Fukashiro et al., 1995b). The subjects were instructed to perform the hopping on one leg with their arms at their sides. The natural jump frequency is around two jumps per second and the subjects were guided to come close to this speed when performing the practice trials (Jones and Watt, 1971, Farley et al., 1991, Funase et al., 2001). It was, however, stressed that the subjects should aim for a comfortable rhythm. The subjects performed approximately 25 jumps and stopped when told by the tester. They first jumped on the right leg, rested for 15 seconds and then jumped on the left leg. The healthy subjects performed three trials, whereas the patients performed two trials on each leg. Any Achilles tendon pain reported after each jump series was documented. The first three and the last two jumps were excluded, which left 20 jumps for the analysis. The following data were used for further analysis: hopping frequency (jumps/second), jump height and the quotient; flight time/contact time, defined in this study as the plyometric quotient.

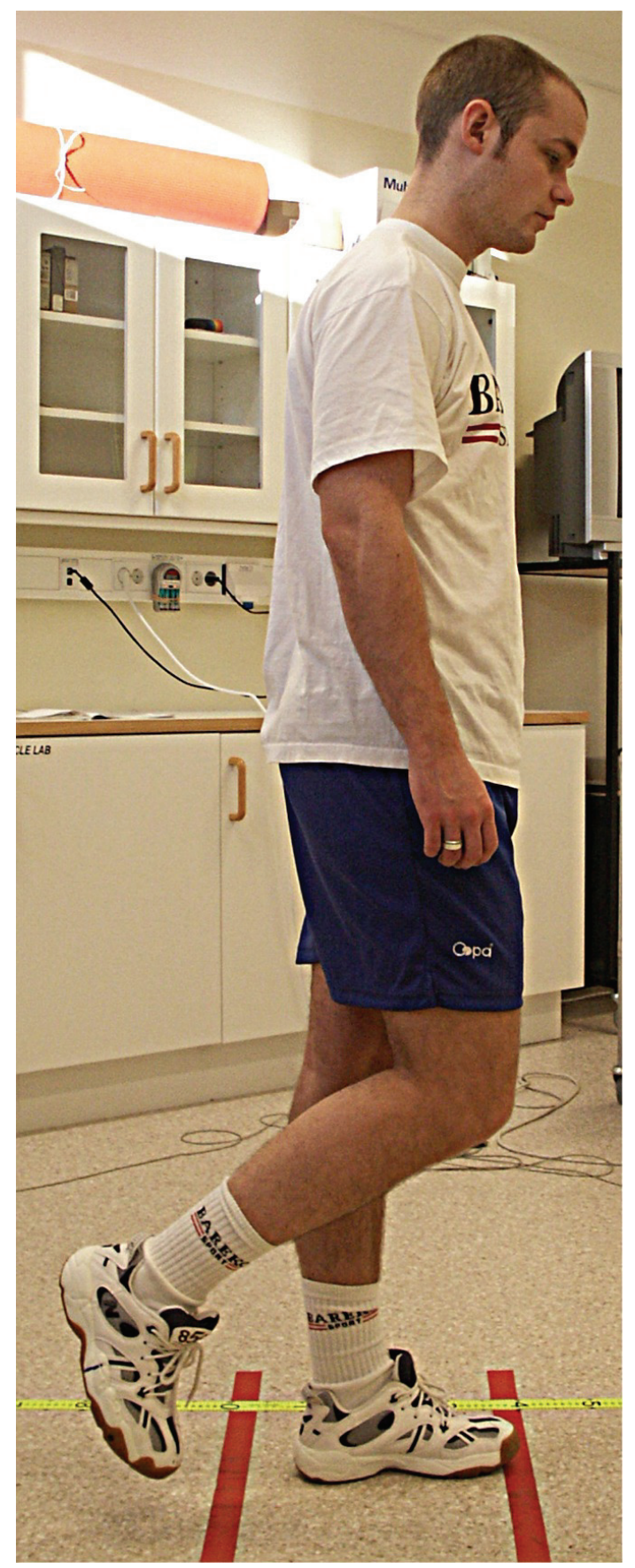

Figure 19. Hopping 
Figure 20. Example of hopping results.

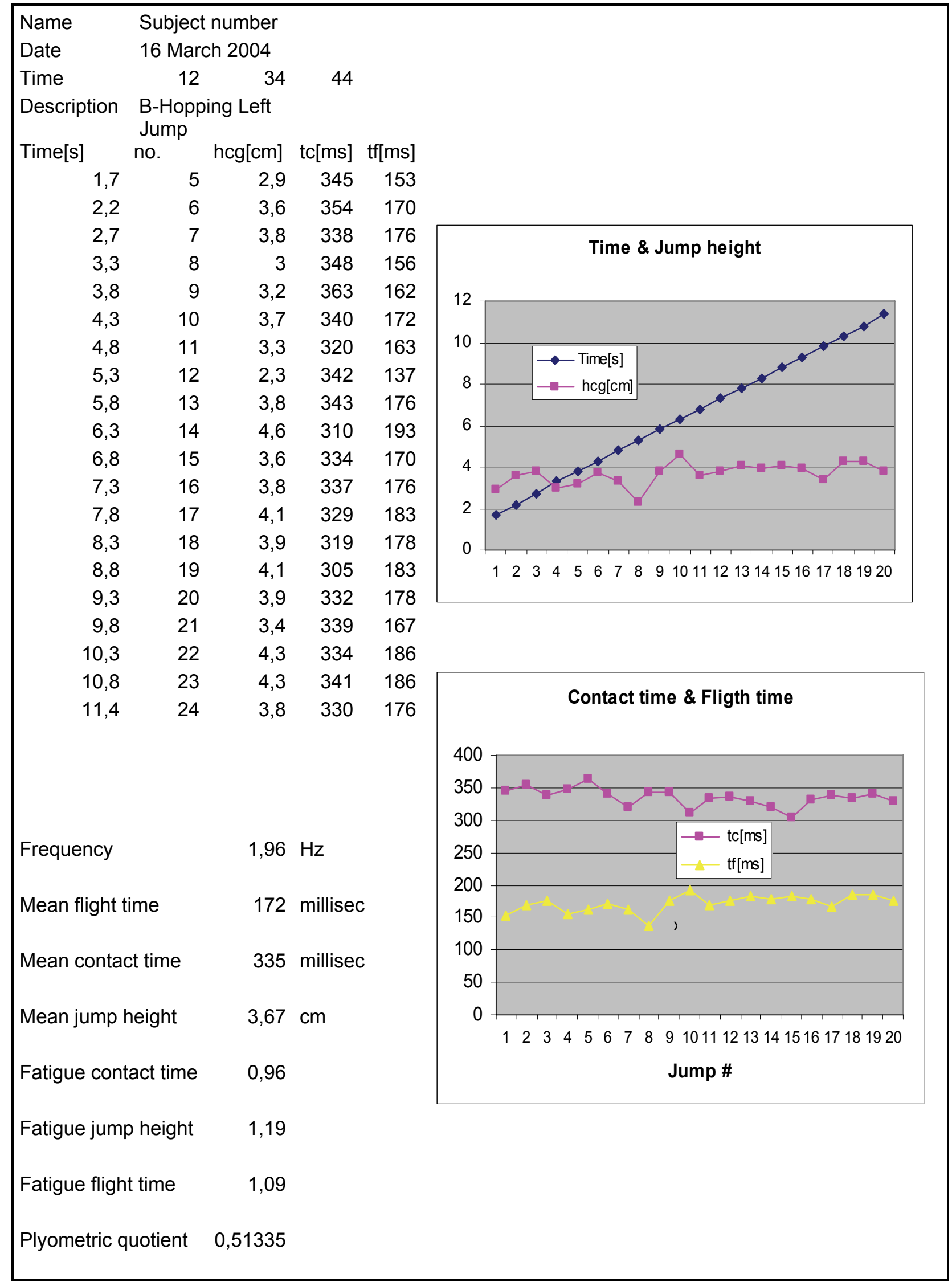




\section{Strength tests}

1. Concentric toe-raises (Figure 21). A concentric toe-raise test was performed standing on one leg in a weight machine. The subjects performed three trials at each weight. The healthy subjects were tested with four different weights, $23 \mathrm{~kg}, 33 \mathrm{~kg}, 43 \mathrm{~kg}$ and $53 \mathrm{~kg}$. The patients started with $13 \mathrm{~kg}$ and increased by $10 \mathrm{~kg}$ until a decrease in power output was seen or the patient was unable to perform the test due to discomfort. The subjects had a 15 -second rest between each trial. The starting position was standing in the machine on both legs. The shoes had a mark to indicate where the foot should be placed and the shoulder positions were also standardized. The healthy subjects placed their hands on the hand rest on the machine. It was then noted that, with heavier weights, the subjects were potentially able to use their hands for assistance during the toe-raises. The patients therefore held their hands in front of their face. An electric goniometer was attached to the knee and measured knee angle at the starting and ending positions. The subjects were not allowed to bend their knee more than 20 degrees during the toe-raise. The instructions to the patients were: $\mathbf{1}$. Stand on both feet with the body straight. 2. Lower the heels. 3.
Remove the left leg (or right). 4. Go up on your toes as quickly as you can. The subjects had to stand still prior to the "up" command. The linear encoder was attached to the heel of the shoe. The linear encoder measured the length and time of the heel displacement of the toe-raise. The MuscleLab ${ }^{\circledR}$ calculated the peak power in watts. The best trial, i.e. the trial with the highest power in watts, for each weight was used for data analysis.

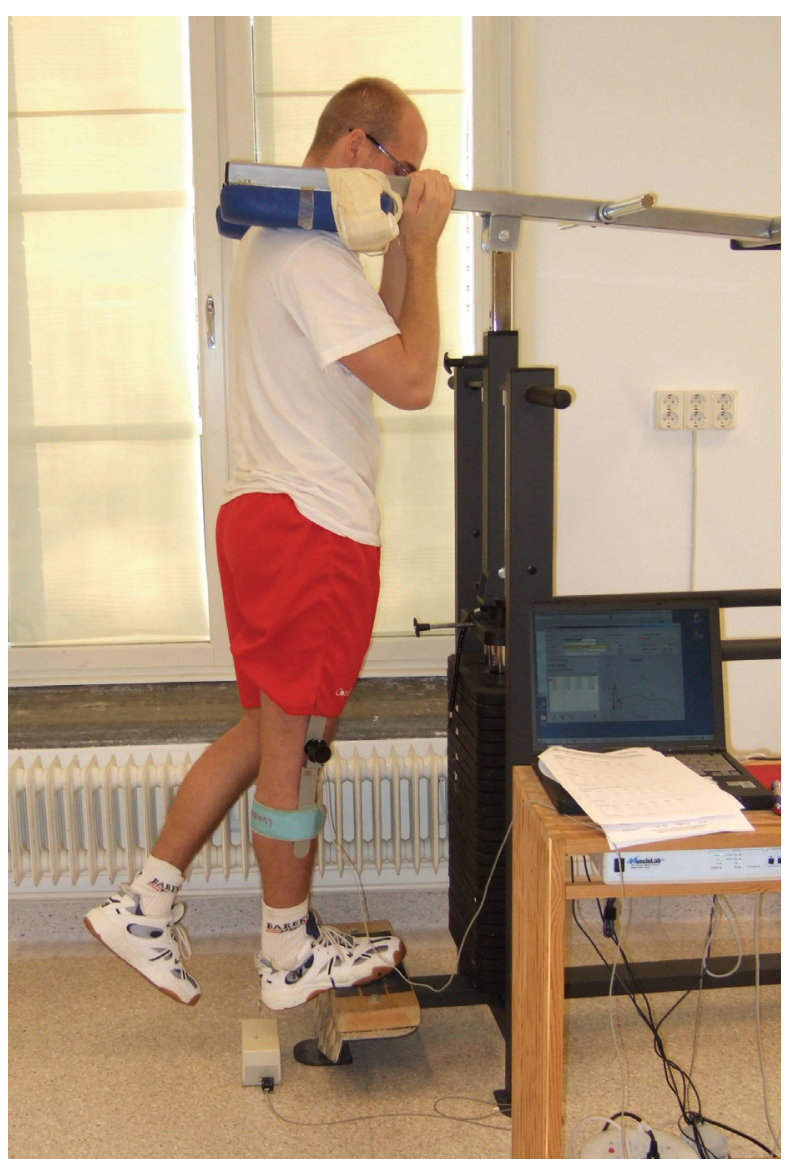

Figure 21. Concentric toe-raises (Starting position) 


\section{Eccentric-concentric toe-raises}

(Figure 22). The eccentricconcentric toe-raise was performed in the same way as the concentric toe-raise except for the starting position in which the foot was in plantar flexion. The subjects were told to go up on their toes on both feet, hold the leg that was not being tested in the air and then move the heel of the leg that was being tested quickly down and then up. The best trial, i.e. the trial with the highest power in watts in the concentric phase, for each weight was used for data analysis.

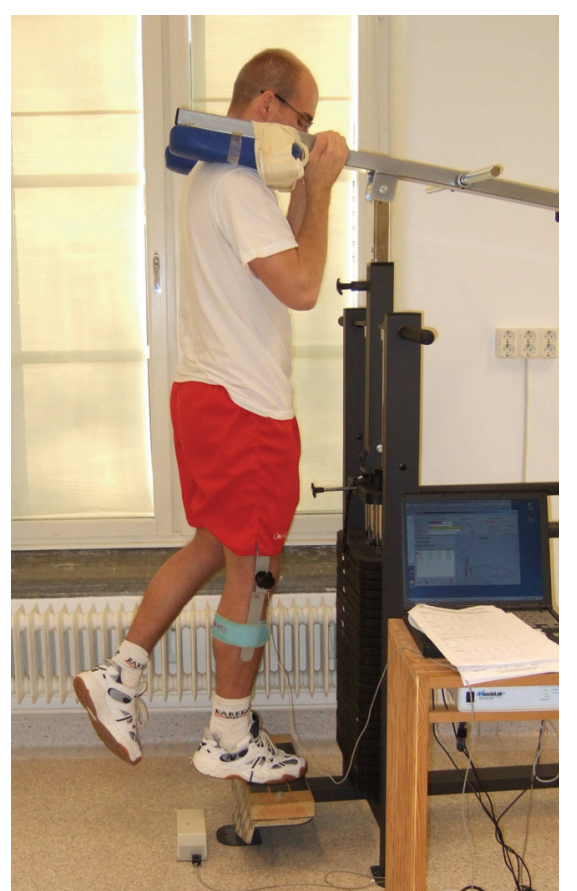

Figure 22. Eccentric-concentric toe-raises (starting position)

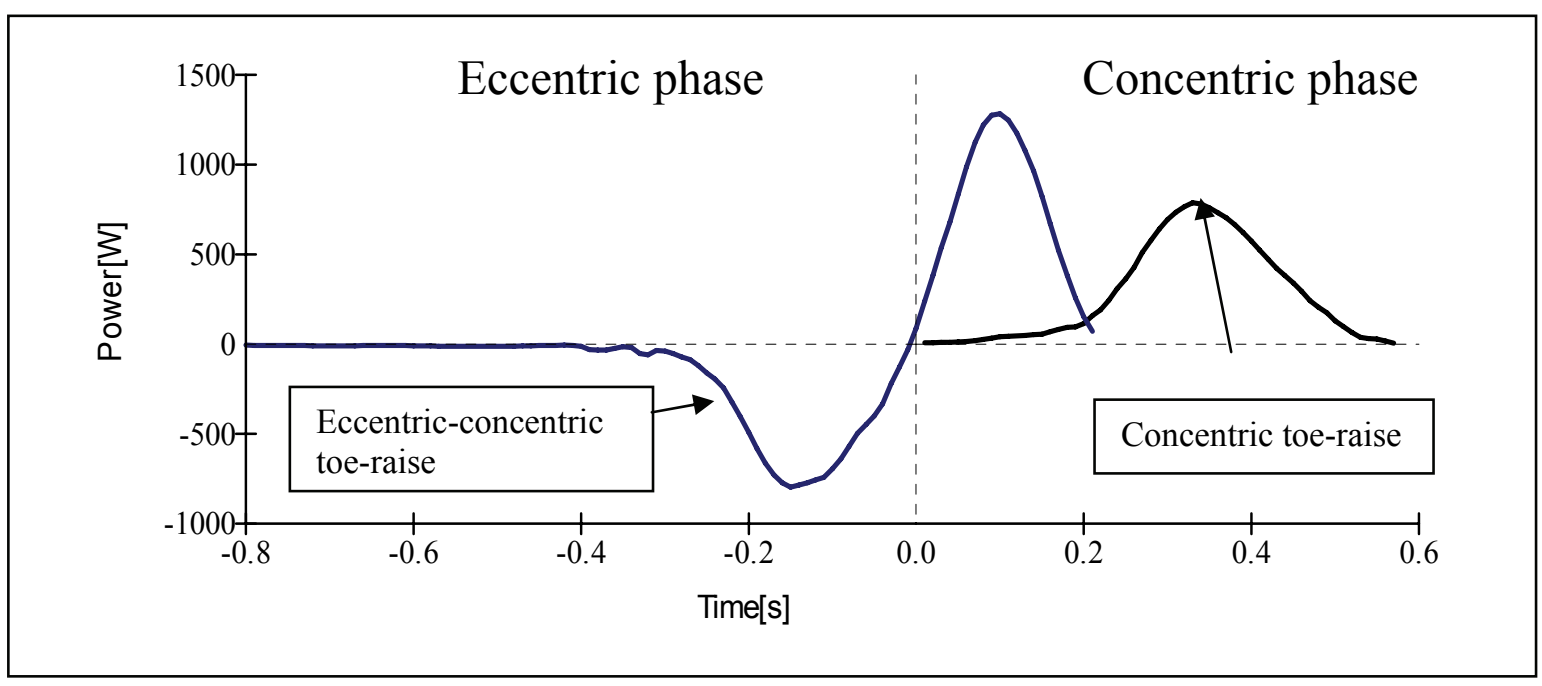

Figure 23. Example of results from a concentric toe-raise and an eccentricconcentric toe-raise

For the healthy subjects the concentric toe-raises for both legs were performed prior to the eccentric-concentric toe-raises. However for the patients, both the concentric and eccentric-concentric toe-raises on one leg were performed prior to switching legs. This was changed for practical reasons because the patients did not perform as many trials as the healthy subjects. 


\section{Muscular endurance test}

\section{Toe-raise test for endurance}

(Figure 24).

The toe-raise test for endurance was performed on one leg at a time with the patient standing on a box with an incline of 10 degrees. The patient was allowed to have two fingertips per hand, placed at shoulder height, against the wall for balance. The patients also had a weight belt with $10 \%$ of their bodyweight strapped around their waist. This made sure that the patient terminated the test due to fatigue and not due to muscular discomfort or lack of motivation. The linear encoder was attached to the heel of the shoe (Figure 24). A cassette player with a recorded voice that said "up" every two seconds was used to maintain the frequency of 30 toe-raises per minute. The patient was instructed to go as high as possible on each toe-raise and then lower the heel to the starting position and wait for the next "up" signal. The patient was asked to perform as many toeraises as possible. The test was terminated when the patient stopped, was unable to maintain the frequency or did not perform a proper toe-raise. The number of toe-raises, as well as the total work (the total load used in the exercise, body weight and added weight, times total distance times gravity) in joules were used for data analysis.

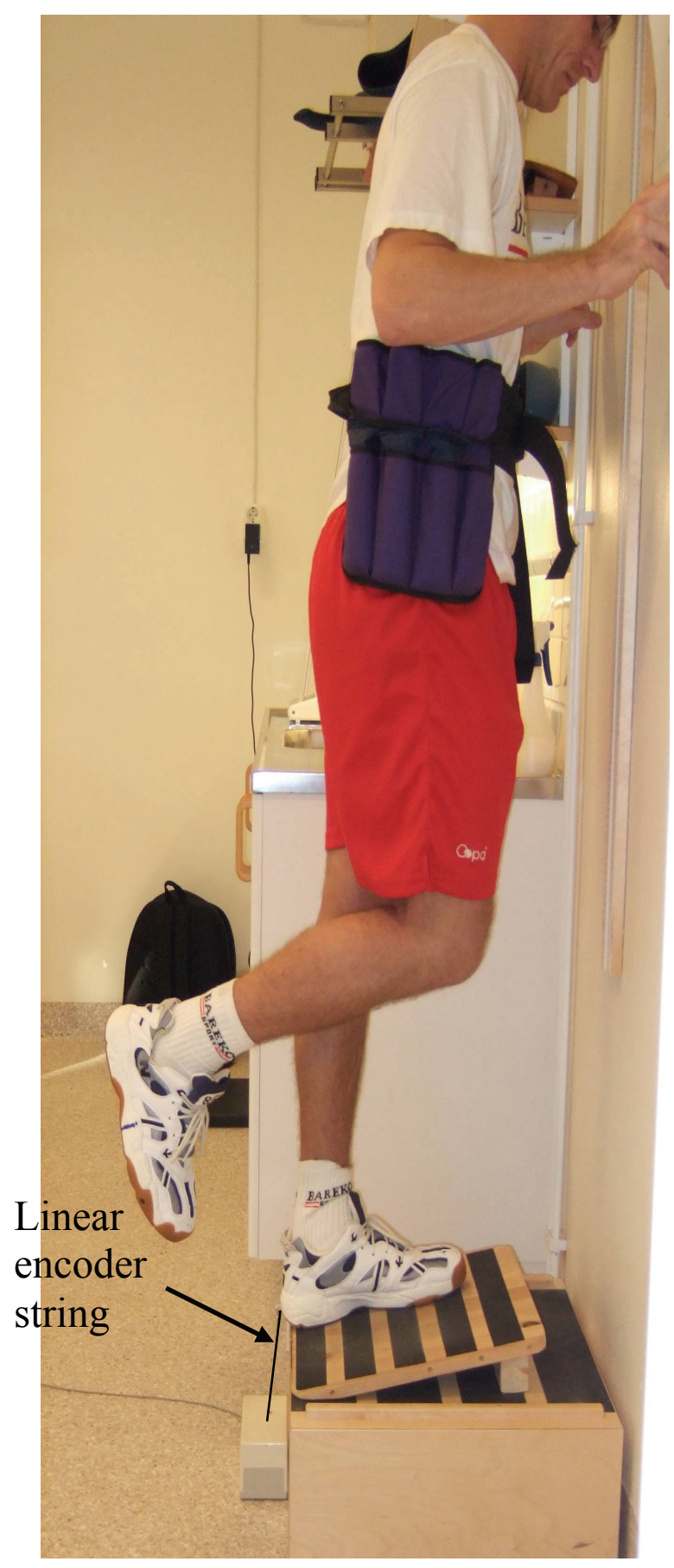

Figure 24. Toe-raise test for endurance 


\section{Treatment}

\section{Study I}

\section{Treatment protocol for the experimental group}

The treatment protocol included information regarding the etiology of the Achilles tendon disorder, a gradually progressive exercise program with weekly supervision by a physical therapist, a pain-monitoring model (Thomeé, 1997) and a diary. The exercise program was divided into three phases.

Phase 1: Days 1-7; included exercises to increase local blood circulation in the lower leg and ankle range of motion, as well as balance and gait exercises and a toe-raise program. The following exercises were used: three sets of 20 repetitions of toe extension/flexion and plantar/dorsal extension/flexion, three sets of $20 \mathrm{~s}$ of calf muscle stretching with the knee extended and calf muscle stretching with the knee flexed, five sets of $30 \mathrm{~s}$ of one leg standing for balance, five sets of $5 \mathrm{~m}$ of walking on toes and walking on heels and two sets of 15 repetitions of regular two-legged concentric/eccentric toe-raises. The patients were told to perform the exercises three times/day.

Phase 2: Weeks 2-3; included the same exercises as phase 1, plus an increase in the toe-raise program, which now consisted of:

a) Two sets of 20 repetitions of regular two-legged concentric/eccentric toeraises.

b) Regular concentric/eccentric toe-raises on one leg. The instructions were to start with 3 sets of 5 repetitions and increase by 2 repetitions each day to 15 repetitions.

c) Eccentric toe-raises on one leg immediately following the regular concentric/eccentric toe raises on one leg, starting with 10 repetitions and increasing by 2 repetitions each day. The instructions were to start with this exercise when able to perform 15 repetitions of regular concentric/eccentric toe-raises on one leg.

d) Stretching the calf muscles for $20 \mathrm{~s}$ afterwards. The patients were told to perform these exercises twice a day.

Phase 3: Weeks 4-12; included the same exercises as phase 2, with a further increase in the toe-raise program, which now consisted of:

a) Two sets of 20 repetitions of regular two-legged concentric/eccentric toeraises.

b) Three sets of 15 repetitions (increasing by 2 repetitions per day if tolerated) of regular one-legged toe-raises on a step, immediately followed by c) 10 
repetitions (increasing by 2 repetitions per day if tolerated) of eccentric toeraises on one leg on a step.

d) Three sets of 20-100 repetitions of quick rebounding toe-raises starting on two legs and progressing to one leg.

e) Stretching the calf muscles for $20 \mathrm{~s}$ afterwards.

During phase 3 , the patients were asked to perform the exercises to increase the local blood circulation in the lower leg, as well as the range of motion, balance and gait exercises once a day and the toe-raise program every other day.

The progress of the exercise program was supervised by a physical therapist and was dependent on the patient's ability and symptoms. The patients were given specific instructions about the exercises at the beginning of each phase. The exercise program was not allowed to increase the patient's symptoms, but pain was allowed during the exercise program according to the pain-monitoring model (Figure 25) (Thomeé, 1997).

\section{Pain-monitoring model}

Visual Analog Scale -VAS

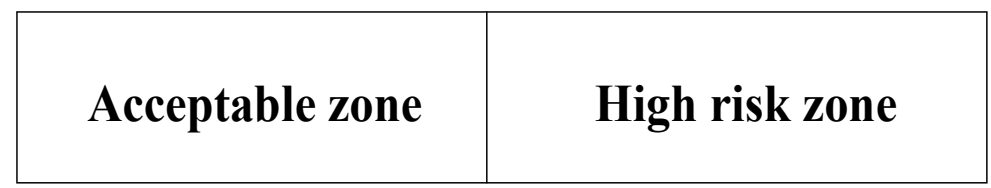

$\begin{array}{lll}0 & 5 & 10\end{array}$

No pain

Worst pain imaginable

1. The pain was allowed to reach 5 on the VAS during the exercises, if the pain decreased immediately after the end of the exercise.

2. The pain after the whole exercise programme was allowed to reach 5 on the VAS but should have subsided the following morning.

3. Pain and stiffness in the Achilles tendon was not allowed to increase from day to day. (Study I)

3. Pain and stiffness in the Achilles tendon was not allowed to increase from week to week. (Study V)

Figure 25. Pain-monitoring model (Thomeé, 1997). 
The exercises were only increased if the above conditions were met and, if not, the patients were told to return to the previous level or contact the treating physical therapist. During the treatment period, the patients kept a training diary in which they recorded their training intensity, any problem related to the exercise program and other physical activities. The experimental group was in touch with the treating physical therapist every week for a follow-up.

\section{Treatment protocol for the control group}

The treatment protocol included brief information regarding the etiology of Achilles tendon disorders, exercises, how the patients should respond to their symptoms and a diary. The treating physical therapist provided information and demonstrated the exercises prior to starting the program. The patients were asked to perform the exercise program three times/day. The exercises included two sets of $30 \mathrm{~s}$ stretching the calf muscle, like the experimental group, and two sets of 30 repetitions of regular two-legged concentric/eccentric toe-raises. The patients were told to progress to three sets of 5 repetitions, with an increase of 2 repetitions a day if possible, of regular concentric/eccentric toe-raises on one leg as soon as the symptoms permitted. The exercise program was not allowed to cause pain or make the symptoms worse and, if this happened, the patients were asked to reduce the training intensity. During the treatment period, the patients kept a training diary and recorded their training intensity, any problem related to the exercise program and other physical activities. The control group was in touch with the treating physical therapist three to five times during the 12 -week treatment period.

\section{Study II}

\section{Treatment with iontophoresis}

Four treatments, with three to four days in-between, with iontophoresis were given during a two-week period, according to the manufacturer's (CEFAR Medical AB, Lund, Sweden) recommendations. The duration of each treatment was approximately 20 minutes. The experimental group was treated with Decadrone and the control group with a saline solution. The study design was double-blinded and neither the patient, the physiotherapist administering the iontophoresis nor the evaluating physical therapist was aware of whether Decadrone or saline solution was used. After the treatment with iontophoresis, both groups followed an identical rehabilitation program for 10 weeks. 


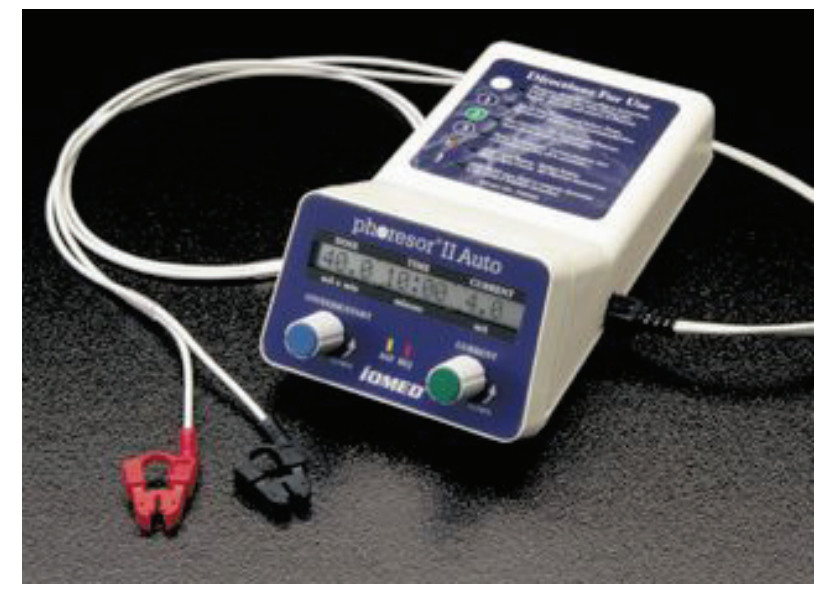

Figure 26. An iontophoresis machine (IOMED by CEFAR Medical AB, Lund, Sweden)

\section{Treatment protocol}

The rehabilitation program included information about the etiology of Achilles tendon pain, a gradually progressive exercises program and a diary. The treating physical therapist provided information and demonstrated the exercises prior to starting the program. The patients were asked to perform the exercise program three times/day. The exercises included two sets of 30 seconds stretching the calf muscle, like the experimental group, and two sets of 30 repetitions of regular two-legged concentricleccentric toe-raises. The patients were told to progress from two-legged toe-raises to three sets of five repetitions, with an increase of two repetitions a day if possible, of regular concentric/eccentric toe-raises on one leg, as soon as the symptoms permitted. The exercise program was not allowed to cause pain or make the symptoms worse and, if this happened, the patients were asked to reduce the training intensity. During the treatment period, the patients kept a training diary and recorded their training intensity, any problem that occurred and was related to the exercise program, as well as other physical activities. The subjects were in touch with the treating physical therapist three to five times during the 10 -week treatment period.

\section{Study V}

\section{Activity criteria for the two groups}

The Exercise training group was allowed to continue Achilles tendon loading activity for the first six weeks of rehabilitation. The patients used the painmonitoring model by Thomeé (1997) as a guide to the level of activity (Figure 25). In accordance to the pain-monitoring model, the pain was allowed to reach level five on the Visual Analogue Scale (VAS) during the exercise 
training. The pain after the exercise program was allowed to reach five on the VAS but should have subsided by the following morning. Pain and stiffness in the Achilles tendon was not allowed to increase from week to week.

The Active rest group was not allowed to perform the activity which caused the symptoms or any other Achilles tendon loading activity involving running or jumping during the first six weeks of rehabilitation. They were not allowed to walk for exercise. They were allowed to swim, run in deep water using a Wet Vest, cycle or walk as daily activity.

\section{Training diaries}

Both groups kept a training diary for 6-12 weeks in which they documented their rehabilitation exercises, other physical activities and symptoms or other comments. Along with the treating physical therapists' documentation, the training diary was used to determine compliance with treatment group.

\section{Treatment protocol}

The Achilles tendon and calf muscle strengthening protocol was based on our previous study but has been modified at the clinic over the years ( Figure 27) (Silbernagel et al., 2001). The exercises were performed once a day and the intensity and number of repetitions were based on the patients' status. The exercises consisted mainly of two-legged, one-legged, eccentric and fastrebounding toe-raises (Figure 28). The intensity was increased gradually by increasing the range of motion (starting with standing on the floor and then performing the exercise standing on stairs), increasing the number of repetitions (starting at 3 sets of maximum amount tolerated up to a maximum of 15 repetitions) and increasing the load (using either a back-pack or weight machine and by increasing the speed of loading). In phase three of the rehabilitation program, the patients started plyometric training. The progression of the exercise program was monitored by the treating physical therapists. 


\section{Figure 27. Treatment protocol}

\section{Phase 1: Weeks 1-2}

Patient status: Pain and difficulty with all activities, difficulty performing 10 one-legged toe-raises

Goal: Start to exercise, understanding of their injury and of pain-monitoring model

Treatment program: Perform exercises every day

- Pain-monitoring model information and advice on exercise activity

- Circulation exercises (moving foot up/down)

- Two-legged toe-raises standing on the floor $(3 \times 10-15)$

- One-legged toe-raises standing on the floor $(3 \times 10)$

- $\quad$ Sitting toe-raises $(3 \times 10)$

- Eccentric toe-raises standing on the floor $(3 \times 10)$

\section{Phase 2: Weeks 2-5}

Patient status: Pain on exercise, morning stiffness, pain when performing toe-raises

Goal: Start strengthening

Treatment program: Perform exercises every day

- Two-legged toe-raises standing on edge of stair $(3 \times 15)$

- One-legged toe-raises standing on edge of stair $(3 \times 15)$

- Sitting toe-raises $(3 \times 15)$

- Eccentric toe-raises standing on edge of stair $(3 \times 15)$

- Quick rebounding toe-raises $(3 \times 20)$

\section{Phase 3: Weeks 3-12 (or longer if needed)}

Patient status: Handle the phase 2 exercise program, no pain distally in tendon insertion, possibly decreased or increased morning stiffness

Goal: Heavier strength training, increase or start running and/or jumping activity

Treatment program: Perform exercises every day and with heavier load 2-3 times per week

- One-legged toe-raises standing on edge of stair with added weight (3x15)

- Sitting toe-raises $(3 \times 15)$

- Eccentric toe-raises standing on edge of stair with added weight (3x15)

- Quick rebounding toe-raises (3x20)

- Plyometric training

Phase 4: Weeks 12-6 months (or longer if needed)

Patient status: Minimal symptoms, not morning stiffness every day, can participate in sports without difficulty

Goal: Maintenance exercise, no symptoms

Treatment program: Perform exercises 2-3 times per week

- One-legged toe-raises standing on edge of stair with added weight (3x15)

- Eccentric toe-raises standing on edge of stair with added weight $(3 \times 15)$

- Quick rebounding toe-raises (3x20) 
Figure 28. The exercises included in the treatment protocol.

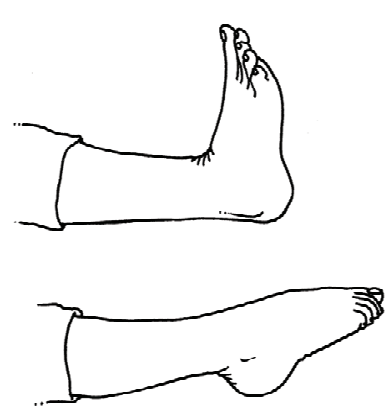

Circulation

exercise

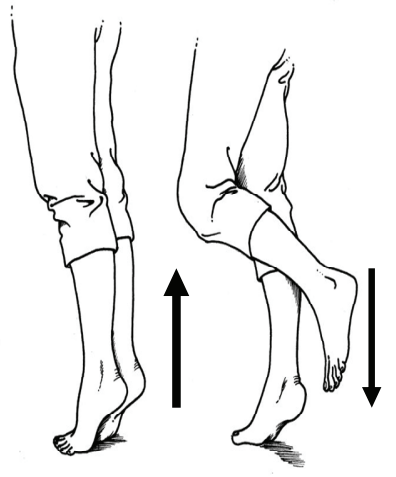

Eccentric toe-raise standing on the floor

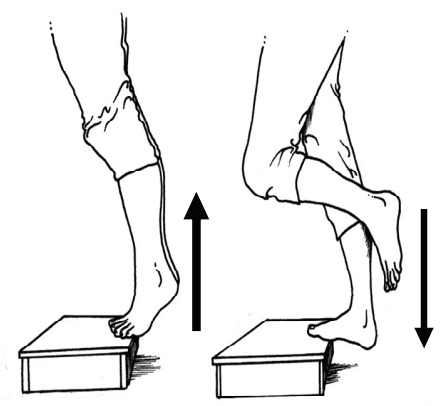

Eccentric toe-raise standing on a step

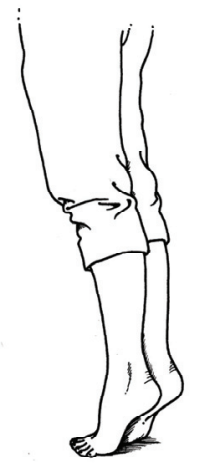

Two-legged toe-raise standing on the floor

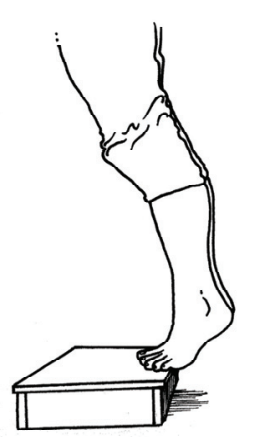

Two-legged toe-raise standing on a step

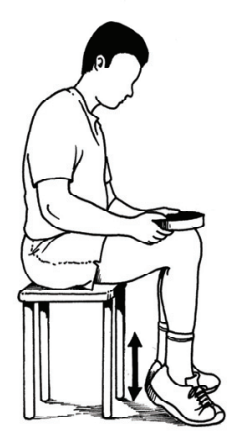

Sitting toe-raise

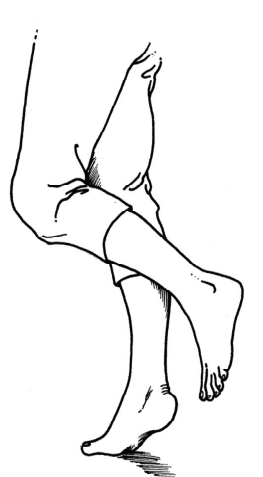

One-legged toe-raise standing on the floor

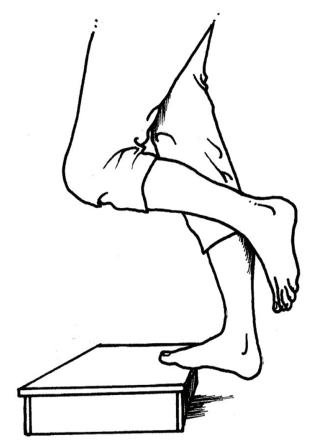

One-legged toe-raise standing on a step

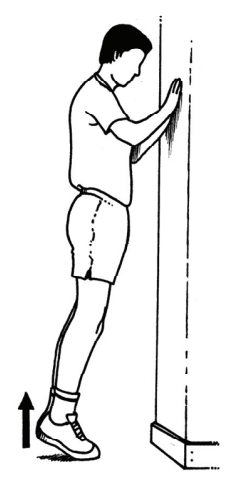

Quick rebounding toe-raise 


\section{Statistical methods}

\section{Study I}

Wilcoxon's Matched-Pairs Signed-Ranks Test and a simple regression analysis were used to evaluate reliability measures. Wilcoxon's MatchedPairs Signed-Ranks test was also used to evaluate the effects of treatment within groups. The Mann-Whitney $U$ test was used to compare groups and Friedman's Two-Way ANOVA Cochran Q test was used to evaluate of questionnaires. When comparing the frequencies of patients with symptoms, Fisher's exact test was used for differences between groups. The significance level was set at $\mathrm{p}<0.05$.

\section{Study II}

Repeated measure ANOVA, Wilcoxon's MPSR T and the Mann-Whitney U test were used to evaluate the effects of treatment. Friedman's two-way ANOVA Cochran Q test was used to evaluate of questionnaires. When comparing the frequencies of patients with symptoms, Fischer's exact test was used for differences between groups. The significance level was $p<0.05$.

\section{Study III}

All the data were analyzed using SPSS 11.5 for Windows. The descriptive data are reported as the mean, standard deviation and $95 \%$ confidence interval (CI). The test-retest data were analyzed using Pearson's $r$, as performed for the VISA-A English version (1986). The Intra-class Correlation Coefficient (ICC) and Wilcoxon's paired test for non-parametric data were also calculated for test-retest data since the questionnaire presents ordinal data. Internal consistency was assessed by calculating Cronbach's alpha.

Comparisons of the VISA-A-S with Stanish and co-workers' (2000) tendon grading system were made by calculating the Spearman's rank correlation coefficient for non-parametric data. VISA-A-S scores for the healthy group and the patient group were compared using the Mann-Whitney $U$ test. For comparisons of the VISA-A-S with the VISA-A, a two-sample ttest was used, since only means and standard deviations, and no raw data, were available from the results in the original study (Murphy et al., 2003). The level of significance was set at $p<0.05$. A principal axis factoring with varimax rotation, eigenvalue over 1.0, was applied to evaluate the structure of the questionnaire.

\section{Study IV}

All the data were analyzed using SPSS 11.5 for Windows. The descriptive data were reported as the mean, standard deviation and range. The test-retest 
data were analyzed with ICC and Wilcoxon's paired test for non-parametric data. The interpretation of the ICC was made according to Fleiss. The methodological error in percent was calculated according to the formula (Dahlberg G):

\section{$[\mathrm{e} / \mathrm{m}] \times \mathbf{1 0 0}=\%$ difference between test ,}

$\mathbf{e}=$ the square root of $\left[\sum\left(d^{2}\right) / 2 n\right]$

$\mathbf{d}=$ difference between tests, $\mathbf{n}=$ number of subjects, $\mathbf{m}=$ mean value

Differences between the "most" symptomatic and "least" symptomatic leg were evaluated using Wilcoxon's signed rank test. Spearman's $r$ was used to evaluate the correlation between the injured leg and the preferred jump leg for patients, as well as between the test battery and patients' symptoms (VISA-A$\mathrm{S}$ score). The level of significance was set at $\mathrm{p}<0.05$. The limb symmetry index (LSI) was calculated to classify a normal or abnormal side-to-side leg difference. The LSI is defined as the ratio of the involved limb score and the uninvolved limb score expressed in percent (involved/uninvolved x $100=$ LSI). In this study, the involved leg was defined as the "most" symptomatic leg and the uninvolved leg was the "least" symptomatic leg or the uninvolved leg. An LSI greater than $90 \%$ was classified as normal. The sensitivity of the individual tests and the test battery was calculated using the following definition: Sensitivity (= number of patients classified as abnormal/total number of patients $\mathrm{x} 100$ ) expresses the percentage probability that the tests will demonstrate an abnormal LSI in patients.

\section{Study V}

A power analysis was carried out prior to the study. It was established that a total of 40 patients were needed to detect a clinically significant mean score difference of 10 points in the VISA-A-S score with $80 \%$ power and at $\mathrm{p}<0.05$. All the evaluations were performed on the basis of an intention-to-treat analysis. This was done to deal safely with non-compliance with the groups. Non-parametric statistics were used, as we could not be sure that the data were normally distributed. To compare the groups, the difference/change between the results from the injured tendons at the initial evaluation and the results at the 6-week, 3-, 6- and 12-month evaluations was used. The groups were then compared using the Mann-Whitney $U$ test. The Mann-Whitney $U$ test was also used to compare the groups at baseline. To evaluate improvements from baseline, Wilcoxon's signed rank test was used. A Spearman's rank correlation coefficient was used to evaluate the correlation between improvement and severity of symptoms and duration of symptoms. 


\section{Summary of studies}

\section{Study I: Eccentric overload training for patients with chronic Achilles tendon pain - a randomised controlled study with reliability testing of the evaluation methods}

Introduction: The purpose was to examine the reliability of measurement techniques and evaluate the effect of a treatment protocol including eccentric overload for patients with chronic pain from the Achilles tendon.

Methods: Thirty-two patients with proximal achillodynia (44 involved Achilles tendons) participated in tests for reliability measures. To evaluate the effect of a 12-week treatment protocol for patients with chronic proximal achillodynia (pain longer than three months) 40 patients (57 involved Achilles tendons) with a mean age of 45 years (range 19-77) were randomised into an experiment group $(n=22)$ and a control group $(n=18)$. Evaluations were performed after six weeks of treatment and after three and six months. The evaluations (including the pre-tests), performed by a physical therapist unaware of the group the patients belonged to, consisted of a questionnaire, a range of motion test, a jumping test, a toe-raise test, a pain on palpation test and pain evaluation during jumping, toe-raises and at rest. A follow-up was also performed after one year.

Results: No significant differences and strong $(r=0.56-0.72)$ or very strong $(r=0.90-0.93)$ correlations were found between pre-tests, except for the documentation of pain at rest $(P<0.008, r=0.45)$. There were no significant differences between groups at any of the evaluations, except that the experiment group jumped significantly lower than the control group at the six-week evaluation. There was, however, an overall better result for the experiment group with significant improvements in plantar flexion, and reduction in pain on palpation, number of patients having pain during walking, having periods when asymptomatic and having swollen Achilles tendon. The controls did not show such changes. Furthermore, at the one-year follow-up there were significantly more patients in the experiment group, compared with the control group, that were satisfied with their present physical activity level, considered themselves fully recovered, and had no pain during or after physical activity.

Conclusion: The measurement techniques and the treatment protocol with eccentric overload used in the present study can be recommended for patients with chronic pain from the Achilles tendon. 


\section{Study II: Iontophoresis with or without dexamethazone in the treatment of acute Achilles tendon pain}

Introduction: It is estimated that $30-50 \%$ of all sports injuries are overuse injuries. Achilles tendon disorders are considered to be one of the most common overuse injuries in elite and recreational athletes. The purpose of this study was to evaluate the effects of iontophoresis with dexamethazone to iontophoresis with saline solutions on patients who had acute (less than 3 months) pain from the Achilles tendon, in terms of range of motion, muscular endurance, pain and symptoms.

Methods: Twenty-five patients (15 men and 10 women), age between 18 and 76 years (mean $=38)$, were evaluated before and after 2 weeks of treatment with iontophoresis, as well as after 6 weeks, 3 and 6 months and 1 year. The patients were divided into two groups, and both were treated for two weeks with iontophoresis. The experiment group $(n=14)$ was treated with $3 \mathrm{ml}$ of dexamethazone and the control group $(\mathrm{n}=11)$ was treated with $3 \mathrm{ml}$ of saline solution. Both groups then followed the same rehabilitation program for 10 weeks.

Results: Good reliability was found for the toe-raise and range of motion tests. Poor reliability was, however, found for the pain on palpation test, which was therefore excluded. No differences were found between or within groups for the toe-raise test. Several significant improvements were seen in the experiment group but not in the control group, in the range of motion test, pain during and after physical activity, pain during walking and walking up and down stairs, morning stiffness and tendon swelling.

Conclusion: Even though the small sample size limits the possibilities to draw definite conclusions, we conclude from the present study, using a double-blind, randomized approach and a 1-year follow-up period, that positive effects from ionthophoresis with dexamethazone were found in the treatment of patients with acute Achilles tendon pain. 


\section{Study III: Cross-cultural adaptation of the VISA-A questionnaire, an index of clinical severity for patients with Achilles tendinopathy, with reliability, validity and structure evaluations}

Introduction: Achilles tendinopathy is considered to be one of the most common overuse injuries in elite and recreational athletes and the recommended treatment varies. One factor that has been stressed in the literature is the lack of standardized outcome measures that can be used in all countries. One such standardized outcome measure is the Victorian Institute of Sports Assessment - Achilles (VISA-A) questionnaire, which is designed to evaluate the clinical severity for patients with Achilles tendinopathy. The purpose of this study was to cross-culturally adapt the VISA-A questionnaire to Swedish, and to perform reliability, validity and structure evaluations.

Methods: Cross-cultural adaptation was performed in several steps including translations, synthesis of translations, back translations, expert committee review and pre-testing. The final Swedish version, the VISA-A Swedish version (VISA-A-S) was tested for reliability on healthy individuals $(\mathrm{n}=15)$, and patients $(\mathrm{n}=22)$. Tests for internal consistency, validity and structure were performed on 51 patients.

Results: The VISA-A-S had good reliability for patients $(\mathrm{r}=0.89, \mathrm{ICC}=$ $0.89)$ and healthy individuals $(\mathrm{r}=0.89-0.99, \mathrm{ICC}=0.88-0.99)$. The internal consistency was 0.77 (Cronbach's alpha). The mean [95\% confidence interval] VISA-A-S score in the 51 patients (50 [44-56]) was significantly lower than in the healthy individuals (96 [94-99]). The VISA-A-S score correlated significantly (Spearman's $r=-0.68$ ) with another tendon grading system. Criterion validity was considered good when comparing the scores of the Swedish version with the English version in both healthy individuals and patients. The factor analysis gave the factors pain/symptoms and physical activity.

Conclusions: The VISA-A-S questionnaire is a reliable and valid instrument and comparable to the original version. It measures two factors; pain/symptoms and physical activity, and can be used in both research and the clinical setting. 


\section{Study IV: Evaluation of lower leg function in patients with Achilles tendinopathy}

Introduction: Achilles tendinopathy is considered to be one of the most common overuse injuries in elite and recreational athletes. However, the effect that the Achilles tendinopathy has on patients' physical performance is still unclear. The purpose of this study was to evaluate if Achilles tendinopathy caused functional deficits on the injured side compared with the non-injured side in patients. A test battery comprised of tests for different aspects of muscle-tendon function of the gastrocnemius, soleus and Achilles tendon complex was developed to evaluate lower leg function. The test battery's test-retest reliability and sensitivity (the percent probability that the tests would demonstrate abnormal lower limb symmetry index in patients) were also evaluated.

Materials and methods: The test battery consisted of three jump tests; a counter movement jump (CMJ), a drop counter movement jump (Drop CMJ) and Hopping, and two strength tests; concentric toe-raises, eccentricconcentric toe-raises and toe-raises for endurance. The reliability was evaluated through a test-retest design on 15 healthy subjects. The test battery's sensitivity and possible functional deficits in patients with Achilles tendinopathy were evaluated on 42 patients (19 women and 23 men).

Results: An excellent reliability was found between test days 1-2 and 2-3 for all tests $(\mathrm{ICC}=0.76-0.94)$ except for concentric toe-raise, test 2-3, which had fair reliability $(\mathrm{ICC}=0.73)$. The methodological error ranged from $8-17 \%$. There were significant differences $(p=0.001-0.049)$ between the non-injured (or least symptomatic) side and injured (most symptomatic) side for hopping, drop CMJ, concentric and eccentric-concentric toe-raises, and significant differences $(p=0.000-0.012)$ in the level of pain during CMJ, hopping, and drop CMJ. The sensitivity of the test battery at a $90 \%$ capacity was $88 \%$.

Conclusions: Achilles tendinopathy causes not only pain and symptoms in patients but also apparent impairments in various aspects of lower leg muscletendon function as measured with the test battery. This test battery is reliable and able to detect differences in lower leg function between the injured or "most symptomatic" and non-injured or "least symptomatic" side in patients with Achilles tendinopathy. The test battery has higher demand on patients' function compared with each individual test. 


\section{Study V: Continued sports activity, using a pain-monitoring model, during rehabilitation in patients with Achilles tendinopathy - a randomized controlled study}

Introduction: Achilles tendinopathy is a common overuse injury, especially among athletes involved in activities that include running and jumping. The recommended treatment varies, but the effect of exercise appears to be promising and the consensus appears to be that all patients should be treated with an exercise program. The patients are often recommended to have an initial period of rest from the pain-provoking activity, such as running and jumping. The purpose of this study was to demonstrate whether continued Achilles tendon loading activity, using a pain-monitoring model, during rehabilitation will result in significant improvements in patients' symptoms and function, and whether this training regimen could have any negative effects on the final outcome in comparison with patients resting from such activity.

Materials and Methods: 38 patients (18 women and 20 men; mean age 46 years) with Achilles tendinopathy were randomly allocated to two different treatment groups during the first 6 weeks of rehabilitation. The Exercise training group $(\mathrm{n}=19)$ was allowed, while using a pain-monitoring model, to continue Achilles tendon loading activity, such as running and jumping, whereas the Active rest group $(\mathrm{n}=19)$ had to stop with such activities. Furthermore, all the patients were rehabilitated according to an identical rehabilitation program; a progressive Achilles tendon and calf muscle strengthening program. The primary outcome measures were the Swedish version of the Victorian Institute of Sports Assessment - Achilles questionnaire (VISA-A-S) and the pain level during tendon loading activity. The patients' function was evaluated with three jump tests, two strength tests and a muscular endurance test. The patients were evaluated at baseline and then after six weeks and three, six and twelve months after initiating treatment.

Results: The Exercise training group had a mean (SD) VISA-A-S score of 57 (15.8) at baseline and $85(12.7)$ at the 12-month follow-up $(p<0.01)$. The Active rest group had a mean (SD) VISA-A-S score of 57 (15.7) at baseline and $91(8.2)$ at the 12-month follow-up $(p<0.01)$. No significant differences in the rate of improvement were found between the groups. However, both groups showed significant $(p<0.01)$ improvements, compared with baseline, in the VISA-A-S score and in pain level with Achilles tendon loading activity 
at the six-week and three-, six- and twelve-months evaluations. Moreover, no significant differences in the rate of improvement were found between the groups in any of the functional evaluations. Both groups improved significantly $(\mathrm{p}<0.05)$ in the total amount of work (joules) performed during the toe-raise test performed at all the follow-ups compared with baseline. Furthermore, both groups showed a significant $(\mathrm{p}<0.05)$ improvement in the eccentric-concentric toe-raise test at 6 weeks, but only the Exercise training group had significant improvements at the other follow-ups. The Exercise training group showed significant $(\mathrm{p}<0.05)$ improvements in Drop CMJ height and hopping quotient at the six-month follow-up, not seen in the Active rest group.

Conclusions: There was no significant difference between the treatment groups concerning the main outcome variables. No negative effects could be demonstrated from continuing Achilles tendon loading activity, with the use of a pain-monitoring model, such as running and jumping during treatment. Our treatment protocol for patients with Achilles tendinopathy, which gradually increases the load on the Achilles tendon and calf muscle, monitored by a pain-monitoring model, demonstrated significant symptomatic improvements. A training regimen of continued, pain-monitored, tendon loading physical activity might therefore represent a valuable option for patients with Achilles tendinopathy.

Figure 29. Mean VISA-A-S scores with $95 \% \mathrm{CI}$, at 0 and 6 weeks, 3, 6 and 12 months evaluations.

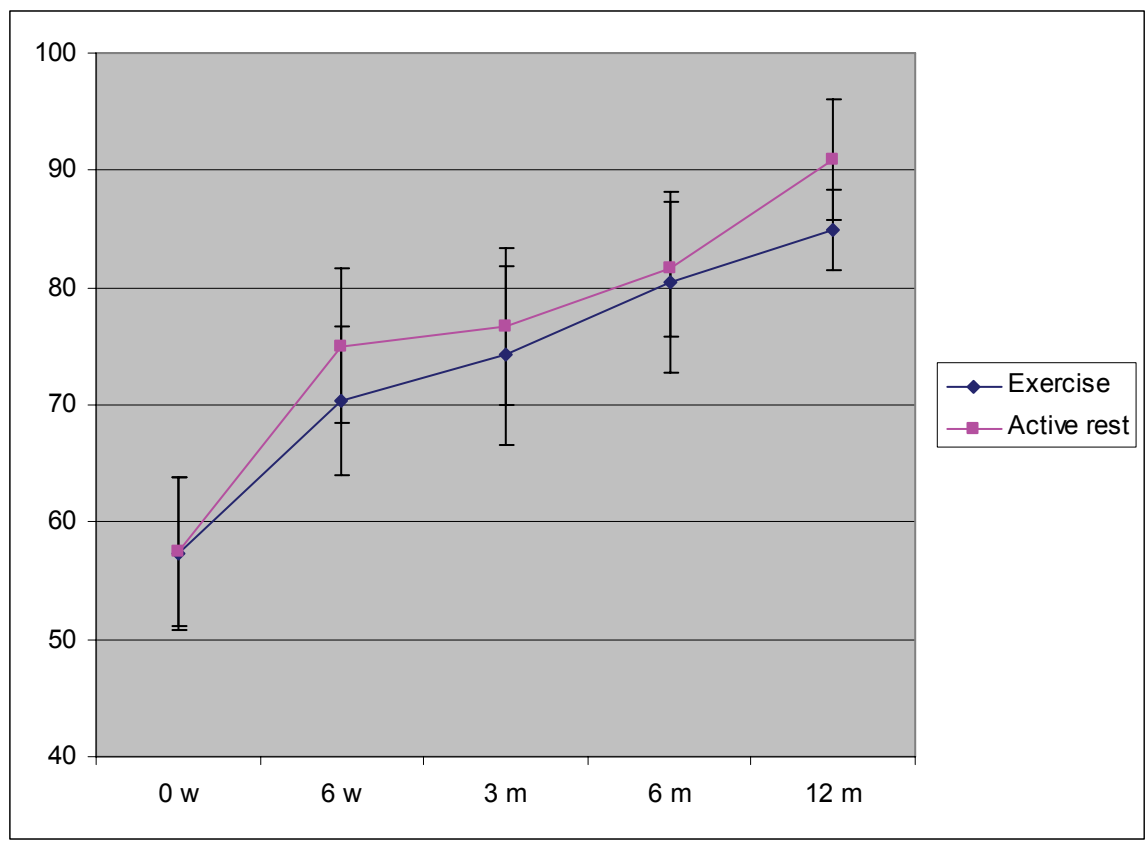




\section{Discussion}

There appears to be consensus in the literature that the term "Achilles tendinopathy" should be the diagnosis for patients with a clinical syndrome, characterized by a combination of pain, swelling (diffuse or localized) and impaired performance.

Achilles tendinopathy can be divided into symptoms from the tendon insertion to the calcaneus or symptoms from the midportion of the tendon. In this thesis, only patients with symptoms from the midportion of the tendon have been included, except for Study III where patients with symptoms from the insertion were also included. The main reason for only studying patients with symptoms from the midportion of the tendon is that the two types of injury differ in structure and injury mechanism and therefore need to be studied separately.

\section{Subjects}

\section{Achilles tendinopathy is a common overuse injury}

Achilles tendinopathy is reported to be a common overuse injury. This was clearly confirmed in this thesis, as there were no difficulties including patients in our studies of long-term injury. In Study I, 40 patients were included during a period of six months, in Study III, 51 patients were included during three months (several clinics around Sweden were involved) and, in Studies IV and V, 42 patients were included during one year.

\section{Physical activity}

The literature states that Achilles tendinopathy causes difficulties with physical activity, something that also was found in the subjects included in this thesis. In Studies IV and V, the patients' pre-injury physical activity level was significantly $(p<0.01)$ higher compared with the physical activity level they had had when entering the study.

The common recommendation of discontinuing Achilles tendon loading activity as part of the treatment probably causes an initial reduction in symptoms, but it may also have negative side-effects on the patient's health. This important negative side-effect of Achilles tendinopathy is not generally discussed in the literature. Today, it is well established that physical activity is important in order to maintain good health. So, any injury causing reduced physical activity needs to be addressed and taken seriously. The recommendation is therefore to start early interventions in order to minimize the negative effects of immobilization and inactivity on the patients. 


\section{Men versus women}

The literature states that Achilles tendinopathy mostly occurs in middle-aged men. In our studies, as well as in others, it can be seen that there is a change over the years in the percentage of women included in the studies. In the often-cited study by Kvist (1991), it was reported that $89 \%$ of patients with Achilles tendinopathy were men. However, this retrospective study only involved athletes and reviewed the years between 1976 and 1986. In a review of several more recent treatment studies the percentage of women was between $14-55 \%$, with the higher percentages seen in the more recent studies (Nelen et al., 1989, Schepsis et al., 1994, Alfredson et al., 1998b, Alfredson et al., 1998c, Angermann and Hovgaard, 1999, Paavola et al., 2000a, Paavola et al., 2000b, Mafi et al., 2001, Öhberg and Alfredson, 2002, Paavola et al., 2002b, Alfredson and Öhberg, 2005d). In a recent treatment study by Roos and co-workers (2004), where the subjects who were included were patients who had sought help at a primary care facility, the percentage of women was $52 \%$.

In Study I, (which was started in 1998) $22 \%$ of the included subjects were women. In Study II, initiated at the same time as Study I, there were $40 \%$ women with acute Achilles tendinopathy. In Study III, which was performed in 2003 with data collected from several clinics in Sweden, there were 37\% women included. In Studies IV and V, initiated in 2004, there were $45 \%$ women. It might therefore be safe to state that Achilles tendinopathy appears to occur more or less equally in both men and women.

The increased prevalence of Achilles tendinopathy in women is probably due to various reasons, such as a generally increased knowledge of the importance of physical activity, more women participating in sports and a greater awareness of Achilles tendinopathy at primary care facilities.

\section{Age}

The inclusion criterion regarding age in Studies I - III was subjects above 18 years of age, while in Studies IV and V we used an age criterion of 20-60 years. The criterion in other treatment studies is also approximately 20-60 years of age (Angermann and Hovgaard, 1999, Mafi et al., 2001, Alfredson and Lorentzon, 2003a, Roos et al., 2004, Costa et al., 2005). The mean ages of the patients in the studies included in this thesis were 38-46 years. When reviewing studies (both surgical and non-surgical) of patients with Achilles tendinopathy, the average age-range is $30-55$ years, with a total range of 17 80 years (Nelen et al., 1989, Leach et al., 1992, Niesen-Vertommen et al., 1992, Schepsis et al., 1994, Alfredson et al., 1998b, Alfredson et al., 1998c, Angermann and Hovgaard, 1999, Paavola et al., 2000a, Paavola et al., 2000b, 
Mafi et al., 2001, Öhberg and Alfredson, 2002, Paavola et al., 2002b, Neeter et al., 2003, Alfredson and Öhberg, 2005d).

\section{Bilateral injury}

In this thesis, patients with bilateral symptoms have been included. It was argued that the results would therefore provide a much more relevant description of the population of patients with Achilles tendinopathy. In Studies I, III, IV and V $29-42 \%$ of the patients had bilateral injuries. Other studies, which also include patients with bilateral injuries have an occurrence of bilateral injury of approximately 30\% (Nelen et al., 1989, Kvist, 1991, Paavola et al., 2002b, Öhberg and Alfredson, 2004a). In an 8-year follow-up of patients with Achilles tendinopathy, it was also found that $41 \%$ of the patients developed symptoms on their previously uninjured side (Paavola et al., 2000a).

\section{Generalizability}

The groups of patients, who were excluded from participating in the studies, to whom the results of this thesis might not be applicable, are elite athletes, persons less then 18 years of age, patients older than 77 years and patients with a systemic disease. This thesis indicates that the main treatment for Achilles tendinopathy is exercise, which is generally a form of treatment with few negative side-effects. It is therefore most probably safe to apply the recommended treatment model to these patients as well. Exercise is often recommended for many types of injury and illness, and could have many positive side-effects on the patients.

\section{Summary}

- Achilles tendinopathy is a common overuse injury.

- Achilles tendinopathy causes patients to reduce their physical activity level.

- Achilles tendinopathy seems equally common in women and men.

- Achilles tendinopathy usually occurs in the 30 to 55 year age range.

- $30 \%$ of the patients with Achilles tendinopathy have bilateral injuries. 


\section{Evaluation}

\section{Diagnosis}

Achilles tendinopathy is a clinical diagnosis for patients with a clinical syndrome, characterized by a combination of pain, swelling (diffuse or localized) in the Achilles tendon and impaired performance. The clinical examination, along with the patient's history, forms the basis of the diagnosis. The onset of symptoms, injury mechanism, possible previous Achilles tendon injury and what makes the symptoms better or worse should be documented. Complaints of morning stiffness are typical. The clinical examination consists of inspection, palpation and the documentation of any swelling, nodular thickening, crepitation, redness and warmth. The location of the pain is also important, since injury to the midsection of the tendon causes tenderness and pain approximately $2-6 \mathrm{~cm}$ proximal to the tendon insertion, while a distal injury causes pain at the tendon insertion.

Evaluation with imaging is not necessary to establish a diagnosis. Imaging can, however, be useful when the diagnosis is questionable or the patient fails to respond to treatment. MRI has been shown to be reliable and valid as an evaluation tool for tendon tissue changes with treatment (Shalabi et al., 2001, Shalabi et al., 2002, Shalabi et al., 2004a, Shalabi et al., 2004b, Shalabi et al., 2004c). In most of our studies, we have chosen not to use either US or MRI since 1) it is not needed to establish a diagnosis, 2) most of the patients seen in the physical therapy clinic have not undergone US or MRI and 3) we have not had the opportunity to use repeated US or MRI for follow-up. The US on the patients in Study IV and V were performed from 7 months prior to 10 months after the patients' inclusion date. The US-finding were used in the prospective Study V to describe the patient population.

\section{Outcome measures}

Due to a lack of standardized and valid outcome measures it has been difficult or even impossible to compare the outcomes from various treatment studies, in patients with Achilles tendinopathy.

The VISA-A questionnaire is the first outcome measure designed specifically for Achilles tendinopathy. It has also been evaluated for reliability and validity. The Swedish version, called the VISA-A-S questionnaire, was found to be reliable and valid in Study III. A factor analysis revealed that the questionnaire evaluated the two factors pain/symptoms and physical activity. The VISA-A-S is a self-administered questionnaire and provides an index of the clinical severity, which is easily followed over time, for patients with Achilles tendinopathy. 
When performing Studies I and II, it was concluded that there was a need for more standardized outcome measures. At that time the VISA-A questionnaire was, however, not available. The VISA-A-S questionnaire was then used in Studies IV and V, and was found to be both useful in the research and a valuable tool in the clinic. It can therefore be recommended that all research studies of patients with Achilles tendinopathy use the VISA-A questionnaire as a main outcome measure. Furthermore, we recommend a difference of 10 points as the limit for clinical relevance.

Further prospective evaluations of the VISA-A questionnaire, along with comparisons with other scores, such as the FAOS, would provide further information about the responsiveness of the VISA-A.

\section{Functional evaluations}

Achilles tendinopathy appears to cause difficulties with physical activities in the active population, but exactly how it affects the lower leg muscle-tendon functions is not fully understood. Muscular strength, power, muscular endurance, flexibility and motor control are important in physical performance. The improvements of these factors is therefore often prescribed in rehabilitation and injury prevention programs for tendon injuries (Hess et al., 1989, Stanish et al., 2000, Cook and Purdam, 2003).

The functional evaluations previously used in treatment studies of patients with Achilles tendinopathy are strength measurements using dynamometry and muscular endurance tests for the calf muscle. In Study I an evaluation method which measured muscular endurance and a CMJ was shown to have good reliability. However, the validity was questioned since only small changes were detected while symptoms improved. Since the meaning of "function" is vague and includes various aspects related to strength, muscular endurance and the ability to utilize the SSC it appears unrealistic to expect that one or two tests will be enough to describe possible functional deficits and improvements with treatment. A test battery which uses a combination of tests, like that used in the present thesis, therefore appears to be more valid for evaluating function. Comparing the function of the injured versus the uninjured leg or "most" versus "least" symptomatic leg using a test battery of reliable, valid and sensitive tests for strength, muscular endurance and plyometric ability could be the first step in the process of determining whether lower leg function is affected by Achilles tendinopathy.

\section{Test battery}

The purpose of the test battery developed in Study IV was to attempt to evaluate the different aspects of the gastrocnemius and soleus muscles and 
Achilles tendon function, such as maximum strength/power, muscular endurance and the ability of the muscle/tendon to utilize the SSC. The three jump tests were chosen to evaluate the ability of the muscle/tendon to utilize the SSC and to evaluate general strength and function. The CMJ has previously been used to evaluate function in patients and Achilles tendon loading in healthy subjects (Komi and Bosco, 1978, Komi et al., 1992, Silbernagel et al., 2001). The loading of the calf muscle-Achilles tendon complex is, however, greater during hopping and this requires the repetitive loading and utilization of the SSC (Komi et al., 1992). A drop CMJ is often used in training to improve jumping ability in athletes and it also places heavy demands on the ability to utilize the SSC (Komi and Bosco, 1978, Viitasalo et al., 1998). So, for the test battery, we chose the three jump tests: CMJ, drop $\mathrm{CMJ}$ and hopping.

The maximum power or strength of the calf muscle has previously been measured in non-functional, non-weight bearing positions that also require expensive non-portable equipment (Niesen-Vertommen et al., 1992, Alfredson et al., 1998b, Möller et al., 2005). The test battery is also designed for use in both clinical and research settings. The tests used to measure muscular strength and power were therefore performed in a regular weight machine for toe-raises. In this machine, together with the MuscleLab ${ }^{\circledR}$ system, it was possible to measure the power out-put from a toe-raise test. Muscle power development, i.e. the ability to produce a high force quickly, is considered to be more important for both sports performance and injury protection, than the ability to produce a high force (Kraemer et al., 2002).

The toe-raise test for muscular endurance has previously been evaluated and shown to be useful in the clinical and research setting for patients with various types of injury including Achilles tendinopathy (Lunsford and Perry, 1995, Svantesson et al., 1998a, Svantesson et al., 1998b, Möller et al., 2001, Silbernagel et al., 2001).

In Study IV, it was shown that the tests used in the test battery were reliable and valid for detecting differences between the "most" and "least" symptomatic leg in patients with Achilles tendinopathy. For the drop CMJ, a drop height of $30 \mathrm{~cm}$ was used for the healthy subjects. Because of the differences found between the test occasions and the subjects' difficulty to become accustomed to the drop height, this height was reduced to $20 \mathrm{~cm}$ for the patients. Viitasalo and co-workers (1998) found that well-trained triplejumpers improved their function with increased jump height whereas active controls instead experienced reduced function. The optimal drop jump height 
varies with each individual and heights above and below this height are associated with reductions in performance (Komi and Bosco, 1978).

The same phenomenon is seen with maximum power output in muscle activations, where the maximum mechanical power output occurs at $30-50 \%$ of maximum strength (Kaneko, 1983, Neeter et al., 2006). For the concentric toe-raise test, there was a variation among the subjects when it came to the added weight that produced the maximum muscle power output. So, for some subjects, the test at $43 \mathrm{~kg}$ involved too high a weight, which could have been one of the reasons for the lower reliability when using this test. For evaluations in the future, it is therefore recommended that the weight should be increased until a decrease in power is seen and the weight that is used for evaluation should be the weight with the highest peak power.

Despite the fact that $40 \%$ of the patients had bilateral symptoms the tests in the test battery were able to discriminate between the lower leg muscletendon function in the "most" symptomatic/injured side and the "least" symptomatic/uninjured side with significant differences in all tests apart from $\mathrm{CMJ}$ height and toe-raise muscular endurance. It appears that the performance in the CMJ test is dependent on the whole leg function and therefore less useful for evaluating lower leg function. The rationale for using the toe-raise muscular endurance test is that the treatment of Achilles tendinopathy always includes some type of toe-raise and the test could therefore be useful in evaluating the effect of treatment (Niesen-Vertommen et al., 1992, Alfredson et al., 1998b, Angermann and Hovgaard, 1999, Mafi et al., 2001, Silbernagel et al., 2001, Roos et al., 2004). The usefulness of the CMJ and toe-raise muscular endurance tests will be re-evaluated in the future, when the responsiveness of the test battery over time compared with changes in symptoms is examined.

The concentric and eccentric-concentric toe-raise test was not always painful for the subjects. It appeared as if many of the patients avoided fully load the eccentric phase of the eccentric-concentric toe-raise test. It was also seen in Study V that the eccentric-concentric strength improved significantly just six weeks after initiating treatment. Even though the test was not necessarily perceived as painful, it could be that there are both local and central inhibitions to loading the tendon fully in the eccentric phase. This might be a useful mechanism for the body to avoid heavy loads on the tendon which might cause tendon rupture. It is indeed possible that this mechanism can partly explain the clinical finding that the majority of patients with complete Achilles tendon ruptures do not have symptoms prior to the injury. The pain might be protective and perhaps the goal of treatment should not be 
to eliminate the pain but to heal the tendon injury and to fully restore all muscle-tendon functions.

The test battery, as described in this thesis, needs to be further evaluated over time and in combination with the VISA-A questionnaire. It can then be determined whether and when the injured leg passes the criterion of $90 \%$ function compared with the healthy leg, and whether the $90 \%$ criterion correlates to improvements in symptoms.

\section{$\underline{\text { Summary }}$}

- Achilles tendinopathy is a clinical diagnosis characterized by a combination of pain, swelling (diffuse or localized), and impaired performance.

- The VISA-A questionnaire evaluates patients' symptoms and their effect on physical activity.

- The VISA-A questionnaire can be used both in research and in the clinic.

- The test battery comprised of tests for different aspects of muscle-tendon function (maximal strength/power, endurance and the muscle-tendon ability to utilize the stretchshortening cycle) of the gastrocnemius, soleus and Achilles tendon complex, places higher demands on patients' function compared with each individual test.

- Achilles tendinopathy causes not only pain and symptoms in patients but also apparent impairments in various aspects of lower leg muscle-tendon function as measured with the ogy $</$ style $></$ keyword $><$ keyword $><$ style treatment programs for Achilles tendinopathy ensure the complete restoration of lower leg muscle-tendon function along with the relief of pain and symptoms. 


\section{Treatment}

Basic tendon research indicates that exercise increases blood circulation and causes the tendon to become larger, stronger and more resistant to injury (Józsa and Kannus, 1997, Kannus et al., 1997c, Langberg et al., 1998, Langberg et al., 1999a, Buchanan and Marsh, 2001). It has also been suggested that mechanical loading, together with stretching of the tendon, promotes the increased reorganization and synthesis of collagen (Kjaer, 2004).

When Study I was started in 1998, only one randomized study had been performed on exercise as rehabilitation for Achilles tendinopathy (NiesenVertommen et al., 1992). Since then, a few more studies of exercise as treatment have been conducted and the current consensus appears to be that all patients should be treated with an exercise program for at least 3-6 months, at least before any other type of treatment is considered (Niesen-Vertommen et al., 1992, Alfredson et al., 1998b, Angermann and Hovgaard, 1999, Alfredson and Lorentzon, 2000, Mafi et al., 2001, Silbernagel et al., 2001, Kader et al., 2002, Alfredson, 2003c, Roos et al., 2004). For all other types of treatment, such as surgery, sclerosing injections, modalities and medications, some type of exercise is also recommended as a complement to the treatment (Schepsis et al., 1994, Paavola et al., 2000b, Testa et al., 2002, Paavola et al., 2002b, Neeter et al., 2003, Alfredson, 2005a). It is therefore important to study which type of exercise is most beneficial and how the exercise should progress in order to achieve positive results for patients with Achilles tendinopathy.

\section{Exercise treatment}

The randomized treatment Studies I and V confirm earlier results that strengthening exercises for the Achilles tendon, gastrocnemius and soleus muscle complex produce significant improvements in symptoms in patients with Achilles tendinopathy (Niesen-Vertommen et al., 1992, Alfredson et al., 1998b, Mafi et al., 2001, Roos et al., 2004). Even though our results appear to be similar to those of other studies of the effect of exercise on Achilles tendinopathy, direct comparisons between treatment studies are difficult due to variations in outcome measures (Niesen-Vertommen et al., 1992, Alfredson et al., 1998b, Mafi et al., 2001, Silbernagel et al., 2001, Roos et al., 2004).

The exercise protocol in Study I was based on the recommendations for tendon rehabilitation made by Curwin and Stanish in the early 1980s (Curwin, 1984). The basis for the exercise program was then to increase the load on the tendon by increasing both the load and the speed of movement and the 
difficulty of the exercise. The exercise protocol in Study V was based on the protocol used for the experimental group in Study I. The exercise protocol has, however, been slightly changed in the clinic during the period between the two studies. The differences in the exercise protocols are 1) the newer program progresses more rapidly in the loading of the tendon, 2) the newer program is performed once a day until phase four, 3) the load is increased by adding external weights and not only by increasing the number of repetitions and 4) the patients are told to continue the exercise protocol for at least six months or longer if needed. In Study I, the experimental group, which trained using the more intense exercise program, obtained better results than the control group. In Study $\mathrm{V}$, the exercise protocol resulted in significant improvements in patients' symptoms already at the six-week evaluation, with continued improvement up to the six-month evaluation. This concurs with earlier recommendations that the initial treatment for Achilles tendinopathy should be exercise based (Niesen-Vertommen et al., 1992, Alfredson et al., 1998b, Angermann and Hovgaard, 1999, Alfredson and Lorentzon, 2000, Mafi et al., 2001, Silbernagel et al., 2001, Kader et al., 2002, Alfredson, 2003c, Roos et al., 2004). The exercise protocol in Studies I and V consisted of both concentric and eccentric types of exercise. We believe that the improvements that were seen were due to the high level of intensity of training, with daily exercises and a gradual increase in the load, causing positive effects on both the muscle and tendon.

Mafi and co-workers (2001) compared concentric and eccentric calf muscle training for patients with Achilles tendinopathy and found similar improvements in their eccentric training group to those seen in both the Exercise training and Active rest groups in Study V. There appeared to be no negative effects from performing strengthening both concentrically and eccentrically as was done in Study V. Mafi and co-workers (2001) appear to have used a lower intensity in the concentric group, not starting strengthening with body weight until week three and without ever increasing the load beyond body weight. We believe that the key factors for improvement, seen in patients with Achilles tendinopathy, are related to both the intensity that is used and the type of loading in the exercises. The underlying effects of exercise are not fully known, but mechanical loading on tendons appears to be important both for the healing process and in improving the strength of the tendons (Kannus et al., 1997c, Riley, 2005, Sarasa-Renedo and Chiquet, 2005).

In several studies, Langberg and co-workers (1999b, 2000, 2001b) have shown that acute exercise activity on tendons in healthy individuals produces 
an increase in the collagen synthesis. It has also been shown that immobilization causes negative effects on tendons (Kannus et al., 1997c, Kubo et al., 2000). Greater strength in the triceps surae musculature has also been reported to be related to a greater ability of the Achilles tendon to store elastic energy (Muraoka et al., 2005). Mechanical loading through exercise on patients with Achilles tendinopathy therefore appears to be important.

Exactly how the exercise program should be optimally designed and how it should progress needs, to be further investigated, however. In order to improve muscle-tendon function and prevent re-injury aspects of motor control, proprioception, strength, malalignment and flexibility may all need to be addressed.

\section{Acute or short-term versus chronic or long-term injury}

Achilles tendinopathy is an overuse type of injury in which the body's reparative capability is exceeded by repetitive micro-trauma. The injury has then been described as being either acute or chronic, depending on how long the patients have had symptoms. In the literature, the term chronic or longterm has been used for Achilles tendinopathy where the patients have had symptoms for more than 4 weeks and up to more than 3 months. In Studies I and II the 3-month cut-off was used. However, due to discussions about the acute phase of the tendon healing process being very short, we changed the criterion to 2 months in the last two studies (Studies IV and V). Interestingly, all the patients included in these two studies reported pain for 3 months or more.

The healing process of the tendon is described as going through three stages, where the acute inflammatory phase will lasts for up to 3-5 days after injury (Enwemeka, 1989b, Leadbetter, 1992, Józsa and Kannus, 1997, Kader et al., 2002). The use of the term "acute injury" implies an inflammatory component where treatment often consists of some type of anti-inflammatory medication. It might, however, be difficult to determine when the term "acute injury" should be used, since the acute inflammatory phase is very short and some patients have a very slow progressive onset of the injury. A better definition of an acute injury is therefore needed. In the same way, further studies of the aspects of tendon healing are also needed.

In Study II, a cut-off of three months of symptoms was used for the inclusion of patients who were considered to have an acute injury. However, the possibility cannot be excluded that some patients had Achilles tendon pain without ongoing inflammation. The results in this study indicated that iontophoresis with dexamethazone had a beneficial effect compared with placebo. It is suggested in that future studies a more precise diagnosis should 
be established in terms of the duration of symptoms and the onset of symptoms. It also appears to be necessary first to evaluate whether the human inflammatory process, as well as the healing process, should be left undisturbed, without any medication, in order to obtain the optimal regeneration of tissue after tendon injury. Studies of the role of exercise in the inflammatory and healing process might also provide a further insight into the optimal treatment of patients with acute Achilles tendon injury.

\section{Treatment protocol}

The treatments in Studies I, II and V are what are usually described as treatment protocols. This indicates that the treatment consists not only of an exercise program, but also of information relating to the injury, training diaries and a pain-monitoring model. It may therefore be difficult to distinguish between the positive results of the exercise and the other areas of the treatment protocol. However, we feel that most clinicians and researchers probably give the patients similar types of information. Even though this is not reported or standardized, it still might influence the results of the research studies. The information given to patients probably has an impact on their compliance and their ability to perform the exercise program. It has been our purpose to fully disclose all our treatment strategies so that the treatment protocol would be as successful when used by others.

\section{The pain-monitoring model}

The pain-monitoring model used in this thesis was initially designed for patients with patello-femoral pain syndrome (Thomeé, 1997). The painmonitoring model is a tool that helps the clinician and patient to determine how the exercises should progress. The exercises used for the treatment of patients with Achilles tendinopathy often cause an initial increase in symptoms. Patients are often afraid of performing physical activity that causes pain in the Achilles tendon due to fear of further injury or a total Achilles tendon rupture. This fear might prevent the patients to load the Achilles tendon sufficiently to cause meaningful adaptive positive changes in the tendon. We have hypothesized that if no pain is allowed during training the load on the Achilles tendon may be too low and the positive benefits of the exercises will fail to appear. Alfredson (2005b) also reports that their eccentric calf muscle training was allowed to cause pain, but they do not specify how much pain was allowed. The pain-monitoring model has been very useful both in our research and in the clinic. The patients report that it helps them to understand how to deal with the pain. 


\section{Rest}

In Study V an attempt was made to answer a question that is frequently asked by many patients with Achilles tendinopathy, which is "Can I continue with running, football, and badminton" or whatever their main physical activity might be.

Historically, a period of rest from the pain-provoking physical activity, which usually means no running and jumping activity, has been recommended when initiating treatment (Angermann and Hovgaard, 1999, Stanish et al., 2000, Paavola et al., 2000a). The recommendations for rest in the literature vaguely describe some type of modified rest without specific scientific evidence to support it (Stanish et al., 2000, Kader et al., 2002, Paavola et al., 2002a). Since most of these patients are very physically active people, too long a period of rest or a reduction in physical activity may have a negative impact on their quality of life and sporting performance.

In Study V, no negative effects could be demonstrated from continuing Achilles tendon loading activity (such as running and jumping) with the use of a pain-monitoring model during treatment. A training regimen of continued, pain-monitored, tendon loading physical activity, during rehabilitation with an Achilles tendon and calf muscle strengthening program, might therefore represent a valuable option for patients with Achilles tendinopathy.

\section{$\underline{\text { Summary }}$}

- A strengthening exercise program for the Achilles tendon and calf musculature results in significant and clinically relevant improvements in patients with Achilles tendinopathy.

- The exercise program can be allowed to cause symptoms.

- The pain-monitoring model is a tool that helps the clinician and patient to handle the pain and determine how the exercises should progress.

- Allowing continued running and jumping activity (with the use of the pain monitoring model) during the rehabilitation can be an option for patients with Achilles tendinopathy. 


\section{Clinical recommendations}

\section{Evaluation}

The VISA-A questionnaire is user friendly and self-administered and can be used in the clinic. The equipment necessary for using the test battery is both affordable and easily utilized in the clinical setting. However, if the MuscleLab ${ }^{\circledR}$ measuring system is not available, the clinician is recommended to use the following methods: the toe-raise test for muscular endurance (the number of toe-raises performed is documented) and hopping (the pain level on a VAS during 20 one-legged hops is documented).

\section{Treatment}

It can be recommended that patients with Achilles tendinopathy perform Achilles tendon and calf muscle strengthening exercises for 6-12 months. Continued Achilles tendon loading physical activity, with the use of a painmonitoring model, during the rehabilitation may be an option for the patients. Achilles tendinopathy is not difficult to treat, but it takes a long time and requires patience from both the patient and the treating clinician. With information and encouragement, the patients can be motivated to perform their exercise program daily for 3-12 months. The possible side-effects of the exercise treatment are a positive effect on general health and improvements in strength and function. 


\section{Limitations}

Before any conclusions were drawn in the studies of this thesis all possible limitations were thoroughly discussed and considered. Limitations regarding: subjects, generalizability, reliability and validity have been discussed above. Methodological limitations that need to be further considered are presented below.

In Study I and V the treatment consists of a protocol and the influence on the results from the different parts of the protocol can not be separated.

In Study II the sample size can be considered somewhat small and the definition of acute Achilles tendinopathy is somewhat vague.

In Study III there is a small sample size and a somewhat long time between the tests for the test-retest reliability evaluation which could have influenced the results. This could explain the significant differences between the testing occasions in two of the questions.

In Study IV the functional deficits seen in patients with Achilles tendinopathy can be explained by the pain as well as possible tendinopathic changes and these two explanations cannot be separated.

In Study $\mathrm{V}$ the lack of significant differences between the study groups may be a type II error due to insufficient number of subjects. The sample size was, however, determined using a power analysis based on the primary outcome variable. A power calculation was not performed on the secondary functional outcomes and therefore conclusions based on the functional results are drawn with caution. 


\section{Conclusions}

- Achilles tendinopathy is a common tendon overuse injury that occurs in all ages but is most common in persons 30-55 years of age. It has previously been described as occurring primarily in men but nowadays it seems equally common in both men and women. The injury is bilateral in approximately $30 \%$ of patients and has a negative impact on the patient's physical activity level. (Studies I-V)

- The VISA-A questionnaire is an index of the clinical severity of Achilles tendinopathy. The VISA-A questionnaire is an easily selfadministered questionnaire, which evaluates symptoms and their effect on physical activity. The VISA-A-S questionnaire is a reliable and valid instrument and is comparable to the original version. It measures two factors, pain/symptoms and physical activity, and can be used both in research and in the clinical setting. (Studies III-V)

- With careful standardization, acceptable reliability in the evaluation methods for patients with Achilles tendinopathy can be obtained. However, the validity for only evaluating ankle range of motion, calf muscle endurance or jumping ability can be questioned, since only small changes occur as the symptoms improve. A test battery that evaluates lower leg function with three jumping tests, two strength tests and a muscular endurance test was shown to be reliable and valid for patients with Achilles tendinopathy. The test battery was able to detect differences in lower leg function between the injured or "most symptomatic" and non-injured or "least symptomatic" side in patients with Achilles tendinopathy. The test battery imposed higher demands on patients' function compared with each individual test. Achilles tendinopathy causes not only pain and symptoms in patients but also apparent impairments in various aspects of lower leg muscle-tendon function as measured with the test battery. It is therefore recommended that treatment programs for Achilles tendinopathy ensure the complete restoration of lower leg muscle-tendon function, along with the relief of pain and symptoms. (Studies I-V)

- Positive effects from using iontophoresis with dexamethazone were found in the treatment of patients with acute Achilles tendon pain. However, it is suggested that future studies, of the effects of 
iontophoresis with dexamethazone, should consider more precisely the length of the pain and symptoms as well as if there is an ongoing inflammation. The question of whether it is beneficial to use antiinflammatory drugs at all or if the human inflammatory process is necessary for the optimal regeneration of tissue after tendon injury also needs to be answered. (Study II)

- Patients with Achilles tendinopathy should be treated with a treatment protocol including exercises which gradually increase the load on the Achilles tendon and calf muscle, along with information about the injury and a pain-monitoring model. The exercise program should be performed daily for 3-12 months and it is allowed to cause increased pain (based on the pain-monitoring model). (Studies I, II and V)

- No negative effects could be demonstrated from continuing Achilles tendon loading activity (such as running and jumping), with the use of a pain-monitoring model during treatment. Our treatment protocol for patients with Achilles tendinopathy, which gradually increases the load on the Achilles tendon and calf muscle, monitored by a painmonitoring model, demonstrated significant symptomatic improvements in both groups. A training regimen of continued, painmonitored, tendon loading physical activity might therefore represent a valuable option for patients with Achilles tendinopathy. (Study V)

\section{To summarize}

Achilles tendinopathy seems to occur equally in both men and women, mostly in the 30- to 55 - year age range and $30 \%$ of the patients have bilateral injuries. The injury also has a negative impact on the patients' physical activity level.

The VISA-A-S questionnaire can be used to evaluate the clinical severity of patients with Achilles tendinopathy and it is useful both in the clinic and in research. Achilles tendinopathy not only causes pain but also affects the patient's lower leg function. In the acute phase, the use of iontophoresis with dexamethazone could be beneficial. For patients with both acute and chronic Achilles tendinopathy it can be recommended to perform Achilles tendon and calf muscle strengthening exercises, with the use of a pain-monitoring model, for 6 months. Patients with Achilles tendinopathy with a duration of more than 3 months may be allowed to continue Achilles tendon loading activity with the use of a pain-monitoring model during the treatment. 


\section{Future}

Several interesting questions have arisen during the work with this thesis. Some of these are:

- What is the mechanism that makes the strengthening exercises produce improvements in patients with Achilles tendinopathy?

- Is it the intensity of the exercises or the type of exercises which are important in producing improvements in patients with Achilles tendinopathy?

- How do improvements in function (as measured using the test-battery) correlate to improvements in symptoms (as measured using the VISAA questionnaire)?

- How does the VISA-A (VISA-A-S) questionnaire compare with other outcome scores, such as the FAOS and Curwin and Stanish tendon grading system?

- What are the long-term effects of the treatment protocol on patients with Achilles tendinopathy?

- How much do the proposed risk factors influence the occurrence of Achilles tendinopathy, especially in older individuals?

- How should patients with distal Achilles tendinopathy be evaluated and treated? 


\section{Abstract in Swedish}

Introduktion: Akilles tendinopati (också kallat hälsenebesvär) är en av de vanligaste överbelastningsskadorna hos elitidrottare och motionärer. Trots detta finns det endast ett fătal randomiserade behandlings-studier. Det finns också behov av standardiserade mätinstrument för utvärdering av patienternas symptom och funktion.

Syfte: Det övergripande syftet med denna avhandling var att utveckla och utvärdera mätinstrument och behandlingsprotokoll för patienter med Akilles tendinopati.

Material, Metod och Resultat: Initialt utvecklades och användes en metod för att utvärdera symptom och funktion i studier I och II. Utvärderingsmetoden visade sig ha god reliabilitet på 10 patienter med akut Akilles tendinopati och 32 patienter med kronisk Akilles tendinopati. Vi ifrågasatte däremot metodens validitet eftersom endast smärre funktionella förändringar upptäcktes samtidigt som symptomen minskade. Vid denna tidpunkt fanns inget standardiserat formulär för utvärdering av symptom hos patienter med Akilles tendinopati. Ett sådant frågeformulär, The Victorian Institute for Sports Assessment - Achilles questionnaire (VISA-A), blev tillgängligt år 2001 på engelska. I studie III kulturanpassades detta formulär till svenska och utvärderades för reliabilitet, validitet och struktur på 15 friska försökspersoner samt 51 patienter. Den svenska versionen av VISA-A frågeformuläret (VISA-A-S) mätte två faktorer, smärta/symptom och fysisk aktivitet, och visade god reliabilitet och validitet samt var jämförbart med original-versionen. VISA-A-S frågeformuläret kan användas både i forskning och i klinisk vardag.

Ett testbatteri för utvärdering av underbenets muskel-sen funktion, innehållande hopp och styrketester, utvecklades och utvärderades i studie IV på 42 patienter. Syftet med testbatteriet var att utvärdera, i mer detalj än vad som varit möjligt tidigare, vadmuskelns och hälsenans funktion och undersöka ifall Akilles tendinopati orsakade funktionsnedsättning på den skadade, jämfört med den friska sidan. Test-batteriet visade sig vara reliabelt och kunde upptäcka kliniska relevanta skillnader mellan den skadade eller den "mest symptomgivande" sidan jämfört med den friska eller "minst symptomgivande" sidan hos patienter med Akilles tendinopati. Testbatteriet ställde högre krav på patientens funktion jämfört med de individuella testerna. 
Behandling med iontofores med dexamethazone tillsammans med rehabiliteringsträning, utvärderades i studie II på patienter med akut Akilles tendinopati. Studie II var en dubbel-blind, randomiserad studie, där 25 patienter inkluderades, som visade att det fanns positiva effekter av iontofores jämfört med en kontroll grupp på patienter med akut Akilles tendinopati.

Hälsene- och vadmuskelbelastande träning (där träningsbelastningen modifierades med hjälp av en smärthanteringsmodell) som behandling för patienter med långvarig (symptom över 2-3 månader) Akilles tendinopati utvärderas på 40 patienter i studie I och på 38 patienter i studie V. Effekten av fortsatt fysisk aktivitet, såsom löpning och hopp, på behandlingsresultatet utvärderades i studie V. Behandlingsprotokollet som inkluderar hälsene- och vadmuskelbelastande träning och en smärthanteringsmodell gav en signifikant förbättring $\mathrm{i}$ patienter med långvarig Akilles tendinopati. Inga negativa effekter kunde påvisas från fortsatt fysisk aktivitet, med löpning och hopp, under rehabiliteringen.

Slutsats: VISA-A-S frågeformuläret kan användas för att i forskningssyfte eller i klinik utvärdera besvärsnivån hos patienter med Akilles tendinopati. Akilles tendinopati orsakar inte bara smärta utan påverkar också muskelsenfunktionen i underbenet. I det akuta skedet kan iontofores med dexamethazone vara värdefullt. För patienter med både akuta och kroniskalångvariga besvär kan det rekommenderas att behandlingen består av träning som belastar vadmuskel och hälsena och en smärthanteringsmodell i sex månader. En behandlingsmodell där fortsatt hälsenebelastande idrottsaktivitet är tillåtet (med hjälp av en smärthanteringsmodell) under rehabiliteringen kan var ett bra alternativ för patienter med Akilles tendinopati. 


\section{Acknowledgements}

I would like to thank each and every one of you, who have helped me, encouraged me and most of all laughed with me during the years of working with this thesis. In particular I would like to thank the following persons:

Roland Thomeé, my mentor, advisor, "boss" and friend. You guided me during the start of my research career and have continued to encourage me throughout all these years. Thanks for all the scientific discussions, the guidance with all the details and making me believe in myself. You made this a fun and great experience.

Jon Karlsson, my second mentor and advisor, you believed I could do this from the very beginning and that kind of support is what helped me finish my $\mathrm{PhD}$. Your encouragement and ability to always be there when I needed it has meant a lot to me. I also want to thank your wife Ingrid for her support and encouragement.

Bengt Eriksson, co-author and critical analyzer, you have always been able to analyze my work from a different angle and ask the difficult questions while at the same time making me feel encouraged and excited about the research.

Björn Rydevik, you were the one who officially accepted me as a $\mathrm{PhD}$ student at the Department of Orthopaedics and you made me feel very welcome.

Linda Johansson, without you a PhD student would be lost when trying to navigate through the jungle of departmental rules, deadlines, forms and unwritten policies. Your support is invaluable.

Michael Möller, thanks for stimulating discussions regarding the Achilles tendon.

My co-authors and colleagues Alexander Gustavsson, Camille Neeter and Pia Thomeé. Thanks for all the support during the research process and for the good times travelling, partying and working together over the years. I am looking forward to reading your $\mathrm{PhD}$ theses.

My research colleague Jesper Augustsson. Thanks for friendly support, good times and most of all for showing me that it is "doable".

My research colleagues Jessika Bolgert Ehrnborg, and Elin Lindh. Thanks for your support and encouragement. Remember, having kids and writing a thesis work well together. 
Annelie Brorson, my colleague and most of all a great friend. Thanks for all the good times, the support during the hard times and most of all for always being there for me. I plan on being there for you during your MSc (and maybe $\mathrm{PhD}$ ?).

Mari Lundberg, without you what would I have done (or who would I have called) during the last year. Having someone going through the $\mathrm{PhD}$ process at the same time and then also having that person being as bright, "goofy" and friendly as you must be a sign of faith. I hope we can do research together in the future but first lets just do "non-scientific stuff ".

Katarina Nilsson, thanks for all the discussions regarding the Achilles tendon. You have become a great friend and your support and encouragement has been very important to me. Now it is your turn...

Karin Larsson and Eva Runesson, thanks for making me feel welcome to the lab, for all the fun discussions and your encouragement over the years. You two make Lundberglab a great work place.

To everybody that has come and gone at Lundberglab over the years, thanks for friendly support and encouragement.

My co-workers at SportRehab - Physical Therapy \& Sports Medicine Clinic, you have all been encouraging and supportive and also made me feel that what I was doing is important to our patients.

The former PT students, now PTs, Andreas Hansson, Patrik Jacobsson, Lena Kjellerup, Håkan Rasmusson, Åsa Swanberg and Dennis Wiberg for their assistance in Studies I, II and IV.

Anette Dahlström for the excellent illustrations.

Gunnar Ekeroth for giving understandable statistical advice.

Jeanette Kliger for excellent revision of the English text on short notice.

Ole J. Olsen for all the technical help with MuscleLab® measurement system.

I also want to thank Alingsås Hospital, Löp och Sko kliniken, Mölndal Hospital Orthopaedic department, and Östra University Hospital Orthopeadic department for referring patients to my research studies. 
To all the patients who have participated in this thesis I want to express my sincere gratitude for giving me your time.

My childhood girlfriends, Anna, Anette, Camilla, Eleonor, Elisabeth, Ewa, Jessika, and Maria; thanks for always being there. Having you all is a privilege and your support, encouragement and friendship from childhood to adult life means everything to me. Where should we travel next?

All my childhood friends from Näset, thanks for all the good times far away from research.

My brothers Svante, Martin and Magnus Grävare, thank you for always believing in me and never doubting in my ability to do what I set my mind on doing. I love you all.

Eva Grävare, my sister in law, who I actually consider as my sister. Thanks for all your support and encouragement during this thesis. You have also been a great friend throughout most of my life.

My nieces and nephews, David, Johanna, Jonathan and Mikaela thanks for being such great and fun "kids". I hope you realize that studying is fun and meaningful and when you are done you can have a party!

My parents, Anne-Marie and Bengt, you are the best parents in the world. You have been my greatest supporters my whole life and you have always made me believe that I can do anything I want. Thanks for the never ending support and encouragement. I hope I can be a parent like you.

Linus and Haylie, my children, you are my everything and I love you

Henry, my husband, you are my best friend and the love of my life. Thanks for your support and never ending trust in me and my abilities. (I need you like the flower needs the rain... America)

\section{Financial Support:}

This thesis has been supported by grants from the The Swedish National Centre for Research in Sports, Vårdalsstiftelsen Research funding and The local Research and Development Council of Gothenburg and Southern Bohuslän. 


\section{References}

Alfredson, H. (2003c) Chronic midportion Achilles tendinopathy: an update on research and treatment. Clin Sports Med, 22, 727-41.

Alfredson, H. (2005a) The chronic painful Achilles and patellar tendon: research on basic biology and treatment. Scand J Med Sci Sports, 15, 252-9.

Alfredson, H. (2005b) Conservative management of achilles tendinopathy: new ideas. Foot Ankle Clin, 10, 321-9.

Alfredson, H., Forsgren, S., Thorsen, K., Fahlström, M., Johansson, H. \& Lorentzon, R. (2001a) Glutamate NMDAR1 receptors localised to nerves in human Achilles tendons. Implications for treatment? Knee Surg Sports Traumatol Arthrosc, 9, 1236.

Alfredson, H. \& Lorentzon, R. (2000) Chronic Achilles tendinosis: recommendations for treatment and prevention. Sports Med, 29, 135-46.

Alfredson, H. \& Lorentzon, R. (2003a) Intratendinous glutamate levels and eccentric training in chronic Achilles tendinosis: a prospective study using microdialysis technique. Knee Surg Sports Traumatol Arthrosc, 11, 196-9.

Alfredson, H., Nordström, P. \& Lorentzon, R. (1998a) Prolonged progressive calcaneal bone loss despite early weightbearing rehabilitation in patients surgically treated for Achilles tendinosis. Calcif Tissue Int, 62, 166-71.

Alfredson, H., Pietilä, T., Jonsson, P. \& Lorentzon, R. (1998b) Heavy-load eccentric calf muscle training for the treatment of chronic Achilles tendinosis. Am J Sports Med, 26, 360-6.

Alfredson, H., Pietilä, T., Öhberg, L. \& Lorentzon, R. (1998c) Achilles tendinosis and calf muscle strength. The effect of short-term immobilization after surgical treatment. Am J Sports Med, 26, 166-71.

Alfredson, H., Thorsen, K. \& Lorentzon, R. (1999) In situ microdialysis in tendon tissue: high levels of glutamate, but not prostaglandin E2 in chronic Achilles tendon pain. Knee Surg Sports Traumatol Arthrosc, 7, 378-81.

Alfredson, H. \& Öhberg, L. (2005c) Neovascularisation in chronic painful patellar tendinosis--promising results after sclerosing neovessels outside the tendon challenge the need for surgery. Knee Surg Sports Traumatol Arthrosc, 13, 74-80.

Alfredson, H. \& Öhberg, L. (2005d) Sclerosing injections to areas of neo-vascularisation reduce pain in chronic Achilles tendinopathy: a double-blind randomised controlled trial. Knee Surg Sports Traumatol Arthrosc, 13, 338-44.

Alfredson, H., Öhberg, L. \& Forsgren, S. (2003b) Is vasculo-neural ingrowth the cause of pain in chronic Achilles tendinosis? An investigation using ultrasonography and colour Doppler, immunohistochemistry, and diagnostic injections. Knee Surg Sports Traumatol Arthrosc, 11, 334-8.

Almekinders, L. C. \& Temple, J. D. (1998) Etiology, diagnosis, and treatment of tendonitis: an analysis of the literature. Med Sci Sports Exerc, 30, 1183-90.

Anderson, F. C. \& Pandy, M. G. (1993) Storage and utilization of elastic strain energy during jumping. J Biomech, 26, 1413-27.

Angermann, P. \& Hovgaard, D. (1999) Chronic Achilles tendinopathy in athletic individuals: results of nonsurgical treatment. Foot Ankle Int, 20, 304-6.

Archambault, J. M., Wiley, J. P., Bray, R. C., Verhoef, M., Wiseman, D. A. \& Elliott, P. D. (1998) Can sonography predict the outcome in patients with achillodynia? J Clin Ultrasound, 26, 335-9. 
Arndt, A. N., Komi, P. V., Bruggemann, G. P. \& Lukkariniemi, J. (1998) Individual muscle contributions to the in vivo achilles tendon force. Clin Biomech (Bristol, Avon), 13, 532-541.

Augustsson, J. \& Thomeé, R. (2000) Ability of closed and open kinetic chain tests of muscular strength to assess functional performance. Scand J Med Sci Sports, 10, 164-8.

Beaton, D. E., Bombardier, C., Guillemin, F. \& Ferraz, M. B. (2000) Guidelines for the process of cross-cultural adaptation of self-report measures. Spine, 25, 3186-91.

Belli, A. \& Bosco, C. (1992) Influence of stretch-shortening cycle on mechanical behaviour of triceps surae during hopping. Acta Physiol Scand, 144, 401-8.

Bleakney, R. R. \& White, L. M. (2005) Imaging of the Achilles tendon. Foot Ankle Clin, 10, 239-54.

Boone, D. C., Azen, S. P., Lin, C. M., Spence, C., Baron, C. \& Lee, L. (1978) Reliability of goniometric measurements. Phys Ther, 58, 1355-90.

Bosco, C., Tarkka, I. \& Komi, P. V. (1982b) Effect of elastic energy and myoelectrical potentiation of triceps surae during stretch-shortening cycle exercise. Int J Sports Med, 3, 137-40.

Boushel, R., Langberg, H., Green, S., Skovgaard, D., Bulow, J. \& Kjaer, M. (2000) Blood flow and oxygenation in peritendinous tissue and calf muscle during dynamic exercise in humans. J Physiol, $524 \mathrm{Pt}$ 1, 305-13.

Buchanan, C. I. \& Marsh, R. L. (2001) Effects of long-term exercise on the biomechanical properties of the Achilles tendon of guinea fowl. J Appl Physiol, 90, 164-71.

Carlsson, A. M. (1983) Assessment of chronic pain. I. Aspects of the reliability and validity of the visual analogue scale. Pain, 16, 87-101.

Carr, A. J. \& Norris, S. H. (1989) The blood supply of the calcaneal tendon. J Bone Joint Surg Br, 71, 100-1.

Clement, D. B., Taunton, J. E. \& Smart, G. W. (1984) Achilles tendinitis and peritendinitis: etiology and treatment. Am J Sports Med, 12, 179-84.

Coleman, B. D., Khan, K. M., Maffulli, N., Cook, J. L. \& Wark, J. D. (2000) Studies of surgical outcome after patellar tendinopathy: clinical significance of methodological deficiencies and guidelines for future studies. Victorian Institute of Sport Tendon Study Group. Scand J Med Sci Sports, 10, 2-11.

Cook, J. L., Khan, K. M., Kiss, Z. S., Purdam, C. R. \& Griffiths, L. (2001) Reproducibility and clinical utility of tendon palpation to detect patellar tendinopathy in young basketball players. Victorian Institute of Sport tendon study group. Br J Sports Med, 35, 65-9.

Cook, J. L. \& Purdam, C. R. (2003) Rehabilitation of lower limb tendinopathies. Clin Sports Med, 22, 777-89.

Costa, M. L., Shepstone, L., Donell, S. T. \& Thomas, T. L. (2005) Shock wave therapy for chronic Achilles tendon pain: a randomized placebo-controlled trial. Clin Orthop Relat Res, 440, 199-204.

Curwin, S. (1984) Tendinitis: its etiology and treatment, Lexington, Mass., Collamore Press.

Davis, W. L., Jr., Singerman, R., Labropoulos, P. A. \& Victoroff, B. (1999) Effect of ankle and knee position on tension in the Achilles tendon. Foot Ankle Int, 20, 126-31.

Demaio, M., Paine, R. \& Drez, D. J., Jr. (1995) Achilles tendonitis. Orthopedics, 18, 195204.

Eastlack, M. E., Axe, M. J. \& Snyder-Mackler, L. (1999) Laxity, instability, and functional outcome after ACL injury: copers versus noncopers. Med Sci Sports Exerc, 31, $210-5$. 
El Hawary, R., Stanish, W. D. \& Curwin, S. L. (1997) Rehabilitation of tendon injuries in sport. Sports Med, 24, 347-58.

Enwemeka, C. S. (1989a) The effects of therapeutic ultrasound on tendon healing. A biomechanical study. Am J Phys Med Rehabil, 68, 283-7.

Enwemeka, C. S. (1989b) Inflammation, cellularity, and fibrillogenesis in regenerating tendon: implications for tendon rehabilitation. Phys Ther, 69, 816-25.

Enwemeka, C. S. (1992) Functional loading augments the initial tensile strength and energy absorption capacity of regenerating rabbit Achilles tendons. Am J Phys Med Rehabil, 71, 31-8.

Farley, C. T., Blickhan, R., Saito, J. \& Taylor, C. R. (1991) Hopping frequency in humans: a test of how springs set stride frequency in bouncing gaits. $J$ Appl Physiol, 71, 2127-32.

Fleiss, J. L. (1986) The design and analysis of clinical experiments, New York, Wiley.

Fredberg, U. (1997) Local corticosteroid injection in sport: review of literature and guidelines for treatment. Scand J Med Sci Sports, 7, 131-9.

Fredberg, U. \& Bolvig, L. (2002) Significance of ultrasonographically detected asymptomatic tendinosis in the patellar and achilles tendons of elite soccer players: a longitudinal study. Am J Sports Med, 30, 488-91.

Frohm, A., Saartok, T., Edman, G. \& Renström, P. (2004) Psychometric properties of a Swedish translation of the VISA-P outcome score for patellar tendinopathy. BMC Musculoskelet Disord, 5, 49.

Fukashiro, S., Komi, P. V., Järvinen, M. \& Miyashita, M. (1995b) In vivo Achilles tendon loading during jumping in humans. Eur J Appl Physiol Occup Physiol, 71, 453-8.

Funase, K., Higashi, T., Sakakibara, A., Imanaka, K., Nishihira, Y. \& Miles, T. S. (2001) Patterns of muscle activation in human hopping. Eur J Appl Physiol, 84, 503-9.

Fyfe, I. \& Stanish, W. D. (1992) The use of eccentric training and stretching in the treatment and prevention of tendon injuries. Clin Sports Med, 11, 601-24.

Galloway, M. T., Jokl, P. \& Dayton, O. W. (1992) Achilles tendon overuse injuries. Clin Sports Med, 11, 771-82.

Giffin, J. R. \& Stanish, W. D. (1993) Overuse tendonitis and rehabilitation. Can Fam Physician, 39, 1762-9.

Gill, S. S., Gelbke, M. K., Mattson, S. L., Anderson, M. W. \& Hurwitz, S. R. (2004) Fluoroscopically guided low-volume peritendinous corticosteroid injection for Achilles tendinopathy. A safety study. J Bone Joint Surg Am, 86-A, 802-6.

Grimby, G. (1986) Physical activity and muscle training in the elderly. Acta Med Scand Suppl, 711, 233-7.

Haglund-Åkerlind, Y. \& Eriksson, E. (1993) Range of motion, muscle torque and training habits in runners with and without Achilles tendon problems. Knee Surg Sports Traumatol Arthrosc, 1, 195-9.

Hansen, P., Aagaard, P., Kjaer, M., Larsson, B. \& Magnusson, S. P. (2003) Effect of habitual running on human Achilles tendon load-deformation properties and crosssectional area. J Appl Physiol, 95, 2375-80.

Harris, P. R. (1982) Iontophoresis: Clinical Research in Musculoskeletal Inflammatory Conditions. The Journal of Orthopaedic and Sports Physical Therapy, 4, 109-112.

Hasson, S. M., Wible, C. L., Reich, M., Barnes, W. S. \& Williams, J. H. (1992) Dexamethasone iontophoresis: effect on delayed muscle soreness and muscle function. Can J Sport Sci, 17, 8-13.

Hess, G. P., Cappiello, W. L., Poole, R. M. \& Hunter, S. C. (1989) Prevention and treatment of overuse tendon injuries. Sports Med, 8, 371-84. 
Humble, R. N. \& Nugent, L. L. (2001) Achilles' tendonitis. An overview and reconditioning model. Clin Podiatr Med Surg, 18, 233-54.

Ingber, D. E. (2005) Tissue adaptation to mechanical forces in healthy, injured and aging tissues. Scand J Med Sci Sports, 15, 199-201.

Ishikawa, M. \& Komi, P. V. (2004) Effects of different dropping intensities on fascicle and tendinous tissue behavior during stretch-shortening cycle exercise. $J$ Appl Physiol, 96, 848-52.

James, S. L., Bates, B. T. \& Osternig, L. R. (1978) Injuries to runners. Am J Sports Med, 6, 40-50.

Jones, D. C. (1998) Achilles tendon problems in runners. Instr Course Lect, 47, 419-27.

Jones, G. M. \& Watt, D. G. (1971) Observations on the control of stepping and hopping movements in man. J Physiol, 219, 709-27.

Józsa, L. \& Kannus, P. (1997) Human tendons. Anatomy, physiology and pathology., Champaign, Human Kinetics.

Järvinen, M. (1992) Epidemiology of tendon injuries in sports. Clin Sports Med, 11, 493504.

Järvinen, T. A., Kannus, P., Maffulli, N. \& Khan, K. M. (2005) Achilles tendon disorders: etiology and epidemiology. Foot Ankle Clin, 10, 255-66.

Järvinen, T. A., Kannus, P., Paavola, M., Järvinen, T. L., Jozsa, L. \& Järvinen, M. (2001) Achilles tendon injuries. Curr Opin Rheumatol, 13, 150-5.

Kader, D., Saxena, A., Movin, T. \& Maffulli, N. (2002) Achilles tendinopathy: some aspects of basic science and clinical management. Br J Sports Med, 36, 239-49.

Kaikkonen, A., Kannus, P. \& Järvinen, M. (1994) A performance test protocol and scoring scale for the evaluation of ankle injuries. Am J Sports Med, 22, 462-9.

Kaneko, M., Fuchimoto, T., Toji, H, Suei, K. (1983) Training effect of different loads on the force-velocity relationship and mechanical power output in human muscle. Scandinavian Journal of Sports Sciences, 5, 50-55.

Kannus, P. (1997a) Etiology and pathophysiology of chronic tendon disorders in sports. Scand J Med Sci Sports, 7, 78-85.

Kannus, P. (1997b) Tendons--a source of major concern in competitive and recreational athletes. Scand J Med Sci Sports, 7, 53-4.

Kannus, P. (2000) Structure of the tendon connective tissue. Scand J Med Sci Sports, 10, 312-20.

Kannus, P. \& Jozsa, L. (1991) Histopathological changes preceding spontaneous rupture of a tendon. A controlled study of 891 patients. J Bone Joint Surg Am, 73, 1507-25.

Kannus, P., Jozsa, L., Natri, A. \& Järvinen, M. (1997c) Effects of training, immobilization and remobilization on tendons. Scand J Med Sci Sports, 7, 67-71.

Kaufman, K. R., Brodine, S. K., Shaffer, R. A., Johnson, C. W. \& Cullison, T. R. (1999) The effect of foot structure and range of motion on musculoskeletal overuse injuries. Am J Sports Med, 27, 585-93.

Khaliq, Y. \& Zhanel, G. G. (2003) Fluoroquinolone-associated tendinopathy: a critical review of the literature. Clin Infect Dis, 36, 1404-10.

Khan, K. M., Cook, J. L., Bonar, F., Harcourt, P. \& Åstrom, M. (1999) Histopathology of common tendinopathies. Update and implications for clinical management. Sports Med, 27, 393-408.

Khan, K. M., Forster, B. B., Robinson, J., Cheong, Y., Louis, L., Maclean, L. \& Taunton, J. E. (2003b) Are ultrasound and magnetic resonance imaging of value in assessment of Achilles tendon disorders? A two year prospective study. Br J Sports Med, 37, 149-53. 
Kirkendall, D. T. \& Garrett, W. E. (1997) Function and biomechanics of tendons. Scand J Med Sci Sports, 7, 62-6.

Kjaer, M. (2004) Role of extracellular matrix in adaptation of tendon and skeletal muscle to mechanical loading. Physiol Rev, 84, 649-98.

Kjaer, M., Langberg, H., Miller, B. F., Boushel, R., Crameri, R., Koskinen, S., Heinemeier, K., Olesen, J. L., Dossing, S., Hansen, M., Pedersen, S. G., Rennie, M. J. \& Magnusson, P. (2005) Metabolic activity and collagen turnover in human tendon in response to physical activity. J Musculoskelet Neuronal Interact, 5, 41-52.

Kjaer, M., Langberg, H., Skovgaard, D., Olesen, J., Bulow, J., Krogsgaard, M. \& Boushel, R. (2000) In vivo studies of peritendinous tissue in exercise. Scand J Med Sci Sports, 10, 326-31.

Kleinman, M. \& Gross, A. E. (1983) Achilles tendon rupture following steroid injection. Report of three cases. J Bone Joint Surg Am, 65, 1345-7.

Knobloch, K., Kraemer, R., Lichtenberg, A., Jagodzinski, M., Gossling, T., Richter, M., Zeichen, J., Hufner, T. \& Krettek, C. (2006) Achilles tendon and paratendon microcirculation in midportion and insertional tendinopathy in athletes. $\mathrm{Am} \mathrm{J}$ Sports Med, 34, 92-7.

Kocher, M. S. \& Zurakowski, D. (2004) Clinical epidemiology and biostatistics: a primer for orthopaedic surgeons. J Bone Joint Surg Am, 86-A, 607-20.

Koenig, M. J., Torp-Pedersen, S., Qvistgaard, E., Terslev, L. \& Bliddal, H. (2004) Preliminary results of colour Doppler-guided intratendinous glucocorticoid injection for Achilles tendonitis in five patients. Scand J Med Sci Sports, 14, 100-6.

Komi, P. V. (2000) Stretch-shortening cycle: a powerful model to study normal and fatigued muscle. J Biomech, 33, 1197-206.

Komi, P. V. \& Bosco, C. (1978) Utilization of stored elastic energy in leg extensor muscles by men and women. Med Sci Sports, 10, 261-5.

Komi, P. V., Fukashiro, S. \& Järvinen, M. (1992) Biomechanical loading of Achilles tendon during normal locomotion. Clin Sports Med, 11, 521-31.

Kongsgaard, M., Aagaard, P., Kjaer, M. \& Magnusson, S. P. (2005) Structural Achilles tendon properties in athletes subjected to different exercise modes and in Achilles tendon rupture patients. $J$ Appl Physiol, 99, 1965-71.

Kraemer, W. J., Adams, K., Cafarelli, E., Dudley, G. A., Dooly, C., Feigenbaum, M. S., Fleck, S. J., Franklin, B., Fry, A. C., Hoffman, J. R., Newton, R. U., Potteiger, J., Stone, M. H., Ratamess, N. A. \& Triplett-Mcbride, T. (2002) American College of Sports Medicine position stand. Progression models in resistance training for healthy adults. Med Sci Sports Exerc, 34, 364-80.

Kubo, K., Akima, H., Kouzaki, M., Ito, M., Kawakami, Y., Kanehisa, H. \& Fukunaga, T. (2000) Changes in the elastic properties of tendon structures following 20 days bedrest in humans. Eur J Appl Physiol, 83, 463-8.

Kujala, U. M., Sarna, S., Kaprio, J., Koskenvuo, M. \& Karjalainen, J. (1999) Heart attacks and lower-limb function in master endurance athletes. Med Sci Sports Exerc, 31, 1041-6.

Kvist, M. (1991) Achilles tendon injuries in athletes. Ann Chir Gynaecol, 80, 188-201.

Kvist, M. (1994) Achilles tendon injuries in athletes. Sports Med, 18, 173-201.

Lagergren, C. \& Lindholm, A. (1959) Vascular distribution in the Achilles tendon; an angiographic and microangiographic study. Acta Chir Scand, 116, 491-5.

Langberg, H., Bulow, J. \& Kjaer, M. (1998) Blood flow in the peritendinous space of the human Achilles tendon during exercise. Acta Physiol Scand, 163, 149-53.

Langberg, H., Bulow, J. \& Kjaer, M. (1999a) Standardized intermittent static exercise increases peritendinous blood flow in human leg. Clin Physiol, 19, 89-93. 
Langberg, H., Olesen, J., Skovgaard, D. \& Kjaer, M. (2001a) Age related blood flow around the Achilles tendon during exercise in humans. Eur J Appl Physiol, 84, 2468.

Langberg, H., Rosendal, L. \& Kjaer, M. (2001b) Training-induced changes in peritendinous type I collagen turnover determined by microdialysis in humans. $J$ Physiol, 534, 297-302.

Langberg, H., Skovgaard, D., Asp, S. \& Kjaer, M. (2000) Time pattern of exercise-induced changes in type I collagen turnover after prolonged endurance exercise in humans. Calcif Tissue Int, 67, 41-4.

Langberg, H., Skovgaard, D., Petersen, L. J., Bulow, J. \& Kjaer, M. (1999b) Type I collagen synthesis and degradation in peritendinous tissue after exercise determined by microdialysis in humans. J Physiol, 521 Pt 1, 299-306.

Leach, R. E., Schepsis, A. A. \& Takai, H. (1992) Long-term results of surgical management of Achilles tendinitis in runners. Clin Orthop Relat Res, 208-12.

Leadbetter, W. B. (1992) Cell-matrix response in tendon injury. Clin Sports Med, 11, $533-$ 78.

Lowdon, A., Bader, D. L. \& Mowat, A. G. (1984) The effect of heel pads on the treatment of Achilles tendinitis: a double blind trial. Am J Sports Med, 12, 431-5.

Lunsford, B. R. \& Perry, J. (1995) The standing heel-rise test for ankle plantar flexion: criterion for normal. Phys Ther, 75, 694-8.

Lysholm, J. \& Wiklander, J. (1987) Injuries in runners. Am J Sports Med, 15, 168-71.

Maffulli, N., Ewen, S. W., Waterston, S. W., Reaper, J. \& Barrass, V. (2000) Tenocytes from ruptured and tendinopathic achilles tendons produce greater quantities of type III collagen than tenocytes from normal achilles tendons. An in vitro model of human tendon healing. Am J Sports Med, 28, 499-505.

Maffulli, N., Kenward, M. G., Testa, V., Capasso, G., Regine, R. \& King, J. B. (2003a) Clinical diagnosis of Achilles tendinopathy with tendinosis. Clin J Sport Med, 13, 11-5.

Maffulli, N., Khan, K. M. \& Puddu, G. (1998) Overuse tendon conditions: time to change a confusing terminology. Arthroscopy, 14, 840-3.

Maffulli, N., Wong, J. \& Almekinders, L. C. (2003b) Types and epidemiology of tendinopathy. Clin Sports Med, 22, 675-92.

Mafi, N., Lorentzon, R. \& Alfredson, H. (2001) Superior short-term results with eccentric calf muscle training compared to concentric training in a randomized prospective multicenter study on patients with chronic Achilles tendinosis. Knee Surg Sports Traumatol Arthrosc, 9, 42-7.

Magnusson, S. P. \& Kjaer, M. (2003) Region-specific differences in Achilles tendon crosssectional area in runners and non-runners. Eur J Appl Physiol, 90, 549-53.

Mahieu, N. N., Witvrouw, E., Stevens, V., Van Tiggelen, D. \& Roget, P. (2005) Intrinsic Risk Factors for the Development of Achilles Tendon Overuse Injury: A Prospective Study. Am J Sports Med.

Mccrory, J. L., Martin, D. F., Lowery, R. B., Cannon, D. W., Curl, W. W., Read, H. M., Jr., Hunter, D. M., Craven, T. \& Messier, S. P. (1999) Etiologic factors associated with Achilles tendinitis in runners. Med Sci Sports Exerc, 31, 1374-81.

Muraoka, T., Muramatsu, T., Fukunaga, T. \& Kanehisa, H. (2005) Elastic properties of human Achilles tendon are correlated to muscle strength. $J$ Appl Physiol.

Murphy, D. F., Connolly, D. A. \& Beynnon, B. D. (2003) Risk factors for lower extremity injury: a review of the literature. Br J Sports Med, 37, 13-29. 
Möller, M., Lind, K., Styf, J. \& Karlsson, J. (2005) The reliability of isokinetic testing of the ankle joint and a heel-raise test for endurance. Knee Surg Sports Traumatol Arthrosc, 13, 60-71.

Möller, M., Movin, T., Granhed, H., Lind, K., Faxen, E. \& Karlsson, J. (2001) Acute rupture of tendon Achillis. A prospective randomised study of comparison between surgical and non-surgical treatment. J Bone Joint Surg Br, 83, 843-8.

Neeter, C., Gustavsson, A., Thomee, P., Augustsson, J., Thomee, R. \& Karlsson, J. (2006) Development of a strength test battery for evaluating leg muscle power after anterior cruciate ligament injury and reconstruction. Knee Surg Sports Traumatol Arthrosc, 1-10.

Neeter, C., Thomeé, R., Silbernagel, K. G., Thomeé, P. \& Karlsson, J. (2003) Iontophoresis with or without dexamethazone in the treatment of acute Achilles tendon pain. Scand J Med Sci Sports, 13, 376-82.

Nelen, G., Martens, M. \& Burssens, A. (1989) Surgical treatment of chronic Achilles tendinitis. Am J Sports Med, 17, 754-9.

Nichols, A. W. (1989) Achilles tendinitis in running athletes. J Am Board Fam Pract, 2, 196-203.

Niesen-Vertommen, S., Taunton, J., Clement, D. \& Mosher, R. (1992) The effect of eccentric versus concentric exercise in the management of Achilles tendonitis. Clin J Sport Med, 2, 109-113.

Norkin, C. C. \& Levangie, P. K. (1983) Joint structure \& function: a comprehensive analysis, Philadelphia, F.A. Davis.

Norkin, C. C. \& White, D. J. (1985) Measurement of joint motion: a guide to goniometry, Philadelphia, Davis.

Noyes, F. R., Barber, S. D. \& Mangine, R. E. (1991) Abnormal lower limb symmetry determined by function hop tests after anterior cruciate ligament rupture. Am J Sports Med, 19, 513-8.

O'brien, M. (1992) Functional anatomy and physiology of tendons. Clin Sports Med, 11, 505-20.

O'brien, M. (1997) Structure and metabolism of tendons. Scand J Med Sci Sports, 7, 55-61.

Paavola, M., Kannus, P., Järvinen, T. A., Khan, K., Jozsa, L. \& Järvinen, M. (2002a) Achilles tendinopathy. J Bone Joint Surg Am, 84-A, 2062-76.

Paavola, M., Kannus, P., Orava, S., Pasanen, M. \& Järvinen, M. (2002b) Surgical treatment for chronic Achilles tendinopathy: a prospective seven month follow up study. Br J Sports Med, 36, 178-82.

Paavola, M., Kannus, P., Paakkala, T., Pasanen, M. \& Järvinen, M. (2000a) Long-term prognosis of patients with achilles tendinopathy. An observational 8-year follow-up study. Am J Sports Med, 28, 634-42.

Paavola, M., Orava, S., Leppilahti, J., Kannus, P. \& Järvinen, M. (2000b) Chronic Achilles tendon overuse injury: complications after surgical treatment. An analysis of 432 consecutive patients. Am J Sports Med, 28, 77-82.

Paavola, M., Paakkala, T., Kannus, P. \& Järvinen, M. (1998) Ultrasonography in the differential diagnosis of Achilles tendon injuries and related disorders. A comparison between pre-operative ultrasonography and surgical findings. Acta Radiol, 39, 612-9.

Paoloni, J. A., Appleyard, R. C., Nelson, J. \& Murrell, G. A. (2004) Topical glyceryl trinitrate treatment of chronic noninsertional achilles tendinopathy. A randomized, double-blind, placebo-controlled trial. J Bone Joint Surg Am, 86-A, 916-22.

Pedersen, B. K. \& Saltin, B. (2006) Evidence for prescribing exercise as therapy in chronic disease. Scand J Med Sci Sports, 16 Suppl 1, 3-63. 
Peers, K. (2003a) Extracorporeal Shock Wave Therapy in chronic Achilles and patellar tendinopathy. Leuven University Press. Leuven, Belgium, Leuven University.

Peers, K. H., Brys, P. P. \& Lysens, R. J. (2003b) Correlation between power Doppler ultrasonography and clinical severity in Achilles tendinopathy. Int Orthop, 27, 1803.

Price, D. D., Mcgrath, P. A., Rafii, A. \& Buckingham, B. (1983) The validation of visual analogue scales as ratio scale measures for chronic and experimental pain. Pain, 17, 45-56.

Puddu, G., Ippolito, E. \& Postacchini, F. (1976) A classification of Achilles tendon disease. Am J Sports Med, 4, 145-50.

Riley, G. P. (2005) Gene expression and matrix turnover in overused and damaged tendons. Scand J Med Sci Sports, 15, 241-51.

Rivenburgh, D. W. (1992) Physical modalities in the treatment of tendon injuries. Clin Sports Med, 11, 645-59.

Robinson, J. M., Cook, J. L., Purdam, C., Visentini, P. J., Ross, J., Maffulli, N., Taunton, J. E. \& Khan, K. M. (2001) The VISA-A questionnaire: a valid and reliable index of the clinical severity of Achilles tendinopathy. Br J Sports Med, 35, 335-41.

Rolf, C. \& Movin, T. (1997) Etiology, histopathology, and outcome of surgery in achillodynia. Foot Ankle Int, 18, 565-9.

Roos, E. M., Brandsson, S. \& Karlsson, J. (2001) Validation of the foot and ankle outcome score for ankle ligament reconstruction. Foot Ankle Int, 22, 788-94.

Roos, E. M., Engström, M., Lagerquist, A. \& Söderberg, B. (2004) Clinical improvement after 6 weeks of eccentric exercise in patients with mid-portion Achilles tendinopathy -- a randomized trial with 1-year follow-up. Scand J Med Sci Sports, 14, 286-95.

Rothstein, J. M., Miller, P. J. \& Roettger, R. F. (1983) Goniometric reliability in a clinical setting. Elbow and knee measurements. Phys Ther, 63, 1611-5.

Sandmeier, R. \& Renström, P. A. (1997) Diagnosis and treatment of chronic tendon disorders in sports. Scand J Med Sci Sports, 7, 96-106.

Sarasa-Renedo, A. \& Chiquet, M. (2005) Mechanical signals regulating extracellular matrix gene expression in fibroblasts. Scand J Med Sci Sports, 15, 223-30.

Schatzker, J. \& Brånemark, P. I. (1969) Intravital observations on the microvascular anatomy and microcirculation of the tendon. Acta Orthop Scand Suppl, 126, 1-23.

Schepsis, A. A., Wagner, C. \& Leach, R. E. (1994) Surgical management of Achilles tendon overuse injuries. A long-term follow-up study. Am J Sports Med, 22, 611-9.

Schunck, J. \& Jerosch, J. (2005) Operative treatment of Haglund's syndrome. Basic, indications, procedure, surgical techniques, results and problems. Foot and Ankle Surgery, 11, 123-130.

Shalabi, A., Kristoffersen-Wiberg, M., Aspelin, P. \& Movin, T. (2001) MR evaluation of chronic Achilles tendinosis. A longitudinal study of 15 patients preoperatively and two years postoperatively. Acta Radiol, 42, 269-76.

Shalabi, A., Kristoffersen-Wiberg, M., Aspelin, P. \& Movin, T. (2004a) Immediate Achilles tendon response after strength training evaluated by MRI. Med Sci Sports Exerc, 36, 1841-6.

Shalabi, A., Kristoffersen-Wiberg, M., Papadogiannakis, N., Aspelin, P. \& Movin, T. (2002) Dynamic contrast-enhanced $\mathrm{mr}$ imaging and histopathology in chronic achilles tendinosis. A longitudinal MR study of 15 patients. Acta Radiol, 43, 198206.

Shalabi, A., Kristoffersen-Wilberg, M., Svensson, L., Aspelin, P. \& Movin, T. (2004b) Eccentric training of the gastrocnemius-soleus complex in chronic Achilles 
tendinopathy results in decreased tendon volume and intratendinous signal as evaluated by MRI. Am J Sports Med, 32, 1286-96.

Shalabi, A., Svensson, L., Kristoffersen-Wiberg, M., Aspelin, P. \& Movin, T. (2004c) Tendon injury and repair after core biopsies in chronic Achilles tendinosis evaluated by serial magnetic resonance imaging. Br J Sports Med, 38, 606-12.

Silbernagel, K. G., Thomeé, R. \& Karlsson, J. (2005) Cross-cultural adaptation of the VISA-A questionnaire, an index of clinical severity for patients with Achilles tendinopathy, with reliability, validity and structure evaluations. $B M C$ Musculoskelet Disord, 6, 12.

Silbernagel, K. G., Thomeé, R., Thomeé, P. \& Karlsson, J. (2001) Eccentric overload training for patients with chronic Achilles tendon pain--a randomised controlled study with reliability testing of the evaluation methods. Scand J Med Sci Sports, 11, 197-206.

Simonsen, E. B., Klitgaard, H. \& Bojsen-Moller, F. (1995) The influence of strength training, swim training and ageing on the Achilles tendon and $\mathrm{m}$. soleus of the rat. $J$ Sports Sci, 13, 291-5.

Soma, C. A. \& Mandelbaum, B. R. (1994) Achilles tendon disorders. Clin Sports Med, 13, 811-23.

Stanish, W. D., Curwin, S. \& Mandell, S. (2000) Tendinitis: its etiology and treatment., New York, Oxford University Press.

Stilwell, D. L., Jr. (1957) The innervation of tendons and aponeuroses. Am J Anat, 100, 289-317.

Svantesson, U., Carlsson, U., Takahashi, H., Thomeé, R. \& Grimby, G. (1998a) Comparison of muscle and tendon stiffness, jumping ability, muscle strength and fatigue in the plantar flexors. Scand J Med Sci Sports, 8, 252-6.

Svantesson, U., Thomeé, R. \& Karlsson, J. (2001) Idrottarens spänstbok - spänst och elasticitet $i$ muskler och senor, SISU idrottsböcker AB.

Svantesson, U., Österberg, U., Thomeé, R. \& Grimby, G. (1998b) Muscle fatigue in a standing heel-rise test. Scand J Rehabil Med, 30, 67-72.

Tallon, C., Coleman, B. D., Khan, K. M. \& Maffulli, N. (2001) Outcome of surgery for chronic Achilles tendinopathy. A critical review. Am J Sports Med, 29, 315-20.

Testa, V., Capasso, G., Benazzo, F. \& Maffulli, N. (2002) Management of Achilles tendinopathy by ultrasound-guided percutaneous tenotomy. Med Sci Sports Exerc, 34, 573-80.

Thermann, H., Frerichs, O., Biewener, A. \& Krettek, C. (2001) Healing of the Achilles tendon: an experimental study. Foot Ankle Int, 22, 478-83.

Thomeé, R. (1997) A comprehensive treatment approach for patellofemoral pain syndrome in young women. Phys Ther, 77, 1690-703.

Tuite, D. J., Renstrom, P. A. \& O'brien, M. (1997) The aging tendon. Scand J Med Sci Sports, 7, $72-7$.

Vailas, A. C., Tipton, C. M., Laughlin, H. L., Tcheng, T. K. \& Matthes, R. D. (1978) Physical activity and hypophysectomy on the aerobic capacity of ligaments and tendons. J Appl Physiol, 44, 542-6.

Van Der Linden, P. D., Van De Lei, J., Nab, H. W., Knol, A. \& Stricker, B. H. (1999) Achilles tendinitis associated with fluoroquinolones. Br J Clin Pharmacol, 48, 4337.

Viitasalo, J. T., Salo, A. \& Lahtinen, J. (1998) Neuromuscular functioning of athletes and non-athletes in the drop jump. Eur J Appl Physiol Occup Physiol, 78, 432-40.

Wilson, J. J. \& Best, T. M. (2005) Common overuse tendon problems: A review and recommendations for treatment. Am Fam Physician, 72, 811-8. 
Visentini, P. J., Khan, K. M., Cook, J. L., Kiss, Z. S., Harcourt, P. R. \& Wark, J. D. (1998) The VISA score: an index of severity of symptoms in patients with jumper's knee (patellar tendinosis). Victorian Institute of Sport Tendon Study Group. J Sci Med Sport, 1, 22-8.

Visnes, H., Hoksrud, A., Cook, J. \& Bahr, R. (2005) No effect of eccentric training on jumper's knee in volleyball players during the competitive season: a randomized clinical trial. Clin J Sport Med, 15, 227-34.

Vora, A. M., Myerson, M. S., Oliva, F. \& Maffulli, N. (2005) Tendinopathy of the main body of the Achilles tendon. Foot Ankle Clin, 10, 293-308.

Åström, M. (1997) On the nature and etiology of chronic achilles tendinopathy. Malmö, Sweden, Lund University.

Åström, M., Gentz, C. F., Nilsson, P., Rausing, A., Sjöberg, S. \& Westlin, N. (1996) Imaging in chronic achilles tendinopathy: a comparison of ultrasonography, magnetic resonance imaging and surgical findings in 27 histologically verified cases. Skeletal Radiol, 25, 615-20.

Åström, M. \& Rausing, A. (1995) Chronic Achilles tendinopathy. A survey of surgical and histopathologic findings. Clin Orthop Relat Res, 151-64.

Åström, M. \& Westlin, N. (1992) No effect of piroxicam on achilles tendinopathy. A randomized study of 70 patients. Acta Orthop Scand, 63, 631-4.

Åström, M. \& Westlin, N. (1994a) Blood flow in chronic Achilles tendinopathy. Clin Orthop Relat Res, 166-72.

Åström, M. \& Westlin, N. (1994b) Blood flow in the human Achilles tendon assessed by laser Doppler flowmetry. J Orthop Res, 12, 246-52.

Öhberg, L. \& Alfredson, H. (2002) Ultrasound guided sclerosis of neovessels in painful chronic Achilles tendinosis: pilot study of a new treatment. Br J Sports Med, 36, 173-5; discussion 176-7.

Öhberg, L. \& Alfredson, H. (2003) Sclerosing therapy in chronic Achilles tendon insertional pain-results of a pilot study. Knee Surg Sports Traumatol Arthrosc, 11, 339-43.

Öhberg, L. \& Alfredson, H. (2004a) Effects on neovascularisation behind the good results with eccentric training in chronic mid-portion Achilles tendinosis? Knee Surg Sports Traumatol Arthrosc, 12, 465-70.

Öhberg, L., Lorentzon, R. \& Alfredson, H. (2001) Neovascularisation in Achilles tendons with painful tendinosis but not in normal tendons: an ultrasonographic investigation. Knee Surg Sports Traumatol Arthrosc, 9, 233-8.

Öhberg, L., Lorentzon, R. \& Alfredson, H. (2004b) Eccentric training in patients with chronic Achilles tendinosis: normalised tendon structure and decreased thickness at follow up. Br J Sports Med, 38, 8-11; discussion 11.

Östenberg, A., Roos, E., Ekdahl, C. \& Roos, H. (1998) Isokinetic knee extensor strength and functional performance in healthy female soccer players. Scand $J$ Med Sci Sports, 8, 257-64. 


\section{Appendices}
A. VISA-A-S questionnaire
B. VISA-A questionnaire 


\section{JßA}

\section{VISA-A-S FRÅGEFORMULÄR - utvärdering av hälsenebesvär}

Namn:

Datum:

Skadad hälsena: Höger / Vänster

\section{DETTA FRÅGEFORMULÄR SYFTAR ORDET SM $\boldsymbol{A} R T A$ SPECIFIKT PÅ SMÄRTA I HÄLSENAN.}

1. När Du stiger upp på morgonen, under hur många minuter upplever du då stelhet $\mathrm{i}$ hälsenan?

$100 \mathrm{~min}$

\begin{tabular}{|c|c|c|c|c|c|c|c|c|c|c|}
\hline $\begin{array}{l}100 \\
\text { min }\end{array}$ & $\begin{array}{c}90 \\
\text { min }\end{array}$ & $\begin{array}{c}\mathbf{8 0} \\
\text { min }\end{array}$ & $\begin{array}{c}70 \\
\text { min }\end{array}$ & $\begin{array}{c}\mathbf{6 0} \\
\text { min }\end{array}$ & $\begin{array}{c}\mathbf{5 0} \\
\text { min }\end{array}$ & $\begin{array}{c}40 \\
\text { min }\end{array}$ & $\begin{array}{c}30 \\
\text { min }\end{array}$ & $\begin{array}{c}\mathbf{2 0} \\
\text { min }\end{array}$ & $\begin{array}{c}10 \\
\text { min }\end{array}$ & $\begin{array}{c}0 \\
\text { min }\end{array}$ \\
\hline 0 & 1 & 2 & 3 & 4 & 5 & 6 & 7 & 8 & 9 & 10 \\
\hline
\end{tabular}

kraftig/ svår smärta

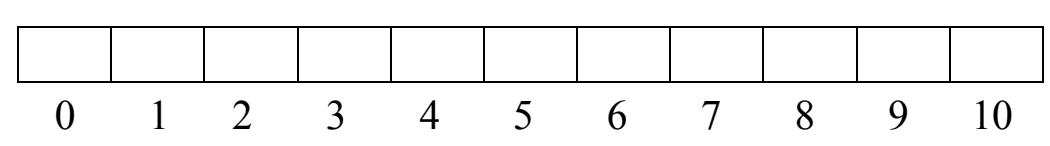

ingen smärta

3. Om du går på plant underlag i 30 minuter, får du då ont i hälsenan inom de närmaste 2 timmarna? (Om du på grund av smärta inte kan gå på plant underlag i 30 minuter, sätt 0 på denna fråga).

kraftig/

svår smärta

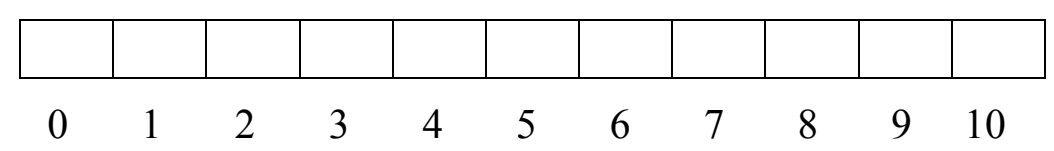

ingen

smärta

4. Får du ont i hälsenan vid normal gång nedför en trappa?

kraftig/ svår smärta

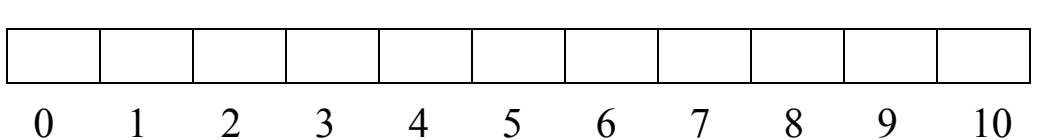

ingen

smärta
Poäng

Poäng smärta

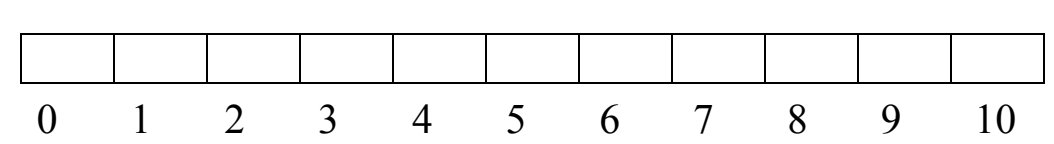

kraftig/ svår smärta

5. Om Du gör 10 tåhävningar (på ett ben) på plant underlag, får du då ont i hälsenan under tiden eller direkt efter?

6. Hur många hopp på ett ben kan du göra utan att få ont i hälsenan?

0

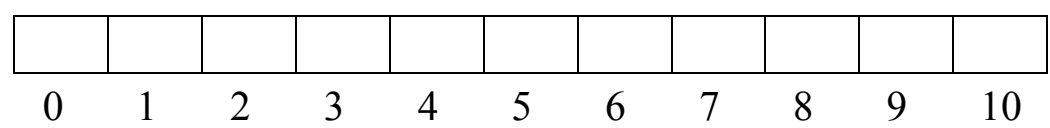


7. Utövar du för närvarande någon idrott eller annan fysisk aktivitet?

0

$\square$ Inte alls

4

Anpassad/begränsad träning och/eller anpassad/begränsad tävling

7

Tränar och/eller tävlar för fullt, men inte på samma nivå som innan hälsenebesvären började.

10

Tävlar på samma nivå eller högre nivå som innan hälsenebesvären började.

8. Besvara antingen $\mathrm{A}, \mathrm{B}$ eller $\mathrm{C}$ i denna fråga.

- Om du inte har någon smärta under aktivitet som belastar hälsenan, besvara endast fråga $\mathbf{A}$.

- Om du har smärta under aktivitet som belastar hälsenan, men smärtan hindrar dig inte från att fullfölja aktiviteten, besvara endast fråga $\mathbf{B}$.

- Om du har smärta som hindrar dig från att slutföra aktivitet som belastar hälsenan, besvara endast fråga $\mathbf{C}$.

A. Om du inte har någon smärta under aktivitet som belastar hälsenan, hur länge kan du då delta i aktiviteten?

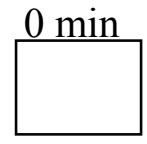

0

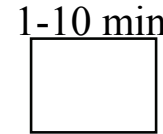

7

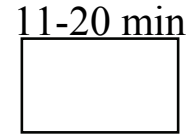

14

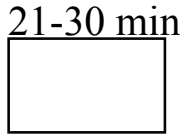

21

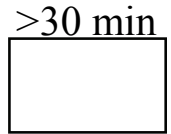

30

ELLER

B. Om du har smärta under aktivitet som belastar hälsenan, men smärtan hindrar dig inte från att fullfölja aktiviteten, hur länge kan du då delta i aktiviteten?
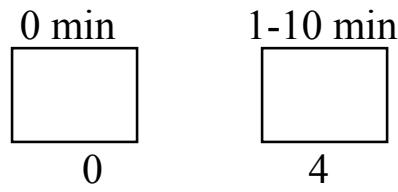

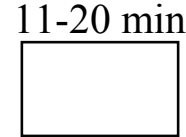

10

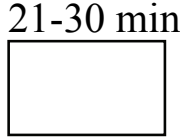

14

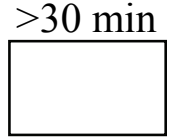

20

Eller

C. Om du har smärta som hindrar dig från att slutföra aktivitet som belastar hälsenan, hur länge kan du då delta i aktiviteten?

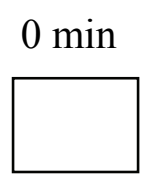

0

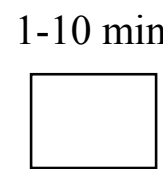

2

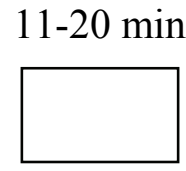

5

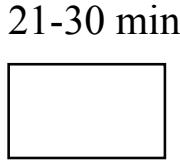

7

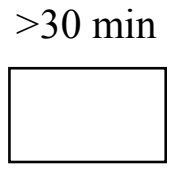

10 